NUREG/CR-6037

\title{
Measurement of Residual Radioactive Surface Contamination by 2-D Laser Heated TLD
}

\author{
DISCLAIMER
}

Manuscript Completed: May 1997

Date Published: June 1997

Prepared by

S.C. Jones

Keithley Instruments, Inc.

28775 Aurora Road.

Cleveland, $\mathrm{OH} 44139$

C. R. Mattsen, NRC Project Manager

Prepared for

Division of Regulatory Applications

Office of Nuclear Regulatory Research

U.S. Nuclear Regulatory Commission

Washington, DC 20555-0001

NRC Job Code W6134

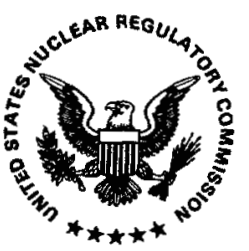

This report was prepared as an account of work sponsored by an agency of the United States Government. Neither the United States Government nor any agency thereof, nor any of their employees, makes any warranty, express or implied, or assumes any legal liability or responsibility for the accuracy, completeness, or usefulness of any information, apparatus, product, or process disclosed, or represents that its use would not infringe privately owned rights. Reference herein to any specific commercial product, process, or service by trade name, trademark, manufacturer, or otherwise does not necessarily constitute or imply its endorsement, recommendation, or favoring by the United States Government or any agency thereof. The vicws and opinions of authors expressed herein do not necessarily state or reflect those of the United States Government or any agency thereof. 


\section{DISCLAMMER}

Portions of this document may be illegible in electronic image products. Images are produced from the best available original document. 


\section{ABSTRACT}

The feasibility of applying and adapting a two-dimensional laser heated thermoluminescence dosimetry system to the problem of surveying for radioactive surface contamination was studied. The system consists of a CO 2 laser-based reader and monolithic arrays of thin dosimeter elements. The arrays consist of 10,201 thermoluminescent phosphor elements of 40 micron thickness, covering a $900 \mathrm{~cm}^{2}$ area. Array substrates are 125 micron thick polyimide sheets, enabling them to easily conform to regular surface shapes, especially for survey of surfaces that are inaccessible for standard survey instruments. The passive, integrating radiation detectors are sensitive to alpha and beta radiation at contamination levels below release guideline limits. Required contact times with potentially contaminated surfaces are under one hour to achieve detection of transuranic alpha emission at $100 \mathrm{dpm} / 100 \mathrm{~cm}^{2}$. Positional information obtained from array evaluation is useful for locating contamination zones. Unique capabilities of this system for survey of sites, facilities and material include measurement inside pipes and other geometrical configurations that prevent standard surveys, and below-surface measurement of alpha and beta emitters in contaminated soils. These applications imply a reduction of material that must be classified as radioactive waste by virtue of its possibility of contamination, and cost savings in soil sampling at contaminated sites. 



\section{TABLE OF CONTENTS}

Abstract

List of Figures

Foreword

Preface

Acknowledgements

\begin{tabular}{l} 
Page \\
\hline iii \\
vi \\
$x i$ \\
$x i i i$ \\
xiv
\end{tabular}

1. Introduction

2. Phase I Feasibility Study

2.1 System Description

2.2 Findings from Phase I Effort

2.3 Discussions and Conclusions for Phase I

3. Phase II Developments and Improvements $\quad 27$

$\begin{array}{ll}3.1 \text { Introduction } & 27\end{array}$

3.2 Phase II Technical Improvements and Developments 28

3.3 Developmental Testing $\quad 40$

3.4 Final Testing and Determination of Detection Limits $\quad 55$

3.5 Phase II Discussions and Conclusions $\quad 82$

4. SBIR Phase III $\quad 85$

$\begin{array}{ll}\text { 5. References } & 87\end{array}$ 


\section{LIST OF FIGURES}

Figure

Page

1 Schematic of the laser TLD reader optical system. The laser is controlled by feedback generated from the split-off beam sample. The optical channel transforms the Gaussian beam into a square of uniform profile. The fiber optic bundle conducts the emitted thermoluminescence to the photomultiplier (PMT) for intensity measurement.

2 Cross-sectional view of TLD array construction.

3 Measurement result following a 30-minute exposure to the depleted ${ }^{238} \mathrm{U}$ alpha source. The activity is $466 \mathrm{dpm}$, over an $8 \mathrm{~mm}$ diameter area. The equivalent specific activity over the array area is $466 \mathrm{dpm} / 54 \mathrm{~cm}^{2}$.

$4 \quad$ Light output for first (above) and second (below) heatings of an alpha irradiated TLD. The difference between the two is the measure of signal. Note that significant signal begins at about $175 \mathrm{msec}$ in the second heating.

5 Plot of signal vs. exposure, in terms of average number of alphas intercepted by an array element. Glow curves were integrated for the full length of the heating cycle.

6 Similar to Fig. 5, except glow curve integration terminates at $175 \mathrm{msec}$. Note that slopes of this plot and Fig. 5 are nearly identical, but the apparent background ( $y$ intercept) is sharply reduced.

7 Resultant measurement following array exposure to $466 \mathrm{dpm}{ }^{238} \mathrm{U}$ alpha source after image processing (low pass filtering). Exposure duration referenced to the contour plot (above): 5 minutes - lower left, 10 minutes - upper left, 20 minutes - upper right, 40 minutes - lower right.

8 Array response to four hour exposure to $9000 \mathrm{dpm}, 25 \mathrm{~mm}$ diameter ${ }^{230} \mathrm{Th}$ alpha source. Array phosphor: $\mathrm{CaSO}_{4}: \mathrm{Tm}$.

9 Array response to six different $1.25 \mathrm{~cm}^{2}$ beta sources. Activities are listed in text. Exposure: $60 \mathrm{~min} . \mathrm{CaSO}_{4}: \mathrm{Tm}$ phosphor.

10 Normalized signal levels (response/source activity) for measurements shown in Fig. 9. Response to ${ }^{147} \mathrm{Pm}$ and ${ }^{14} \mathrm{C}$ is surprisingly high.

11 Result of vertical array exposure to ${ }^{239} \mathrm{Pu}$ contaminated soil. Array was rolled into a cylinder, with overlap for $y>200 \mathrm{~mm}$, preventing alpha exposure. The portion of array below soil surface is $x>180 \mathrm{~mm}$.

12 Array response to $1010 \mathrm{dpm}, 0.5 \mathrm{~mm}$ diameter ${ }^{210} \mathrm{Po}$ needle alpha source. Source was placed $1 \mathrm{~mm}$ above and centered on a TLD element. Exposure time: $15 \mathrm{~min}$.

13 Array response to the ${ }^{210} \mathrm{Po}$ alpha source, placed $7.5 \mathrm{~mm}$ above an interstitial array position. Exposure time: $210 \mathrm{~min}$. 
14 Resulting output of 90-minute exposure to the $466 \mathrm{dpm}{ }^{238} \mathrm{U}$ source, following an approximately uniform 70 mrad ${ }^{60} \mathrm{Co}$ gamma dose delivered to array.

15 Printout of computer display showing glow curves analyzed during an array reading.

16 Readout of unirradiated silicone array after a 50 minute exposure to laboratory air.

17 Readout of unirradiated polyimide array after a 50 minute exposure to laboratory air.

18 Readout of a continuous polyimide/phosphor layer with 1,2, 3, and 4 minute exposures to ${ }^{238} \mathrm{U}$ alpha source. Compare with silicone array readout in Figure 7.

19 Result an array reading given several 2, 8, and 32 minute exposures to the $466 \mathrm{dpm}$ ${ }^{238} \mathrm{U}$ alpha source.

20 Result of processing the data in Figure 19 with a $33 \times 33$ convolution kernel.

21 Result of processing the data in Figure 19 with a $11 \times 11$ convolution kernel.

22 Reading results from 10,20 and 30 minute exposures to the $466 \mathrm{dpm}{ }^{238} \mathrm{U}$ alpha source.

23 Result of processing the data in Figure 22 with a $33 \times 33(10 \mathrm{~cm} \times 10 \mathrm{~cm})$ convolution kernel.

24 Result of processing the data in Figure 22 with a $3 \times 3(0.6 \mathrm{~cm} \times 0.6 \mathrm{~cm})$ convolution kernel.

25 Summarized convolution results. Plotted are the maximum values within the patches of the convoluted source readings.

26 Result from various duration exposures to the $466 \mathrm{dpm}{ }^{238} \mathrm{U}$ alpha source. Note that only one "hot spot" occurs (labeled "?").

27 Map of surface contamination at NTS.

28 Contour plot of an array vertically inserted into soil at NTS. The top $50 \mathrm{~mm}$ of the sheet was removed for a previous experiment. The air/soil boundary was parallel to the $X$ axis at $Y=50 \mathrm{~mm}$.

29 Result of processing Figure 28 with a specialized convolution kernel. The $1 \times 101$ kernel produces a measure of the aggregate activity at a given depth, yielding the activitydepth profile shown.

30 Result of intelligent course/fine reading of $466 \mathrm{dpm}{ }^{238} \mathrm{U}$ alpha exposures.

31 Result of resolution 1 (read every spot) reading of $466 \mathrm{dpm}{ }^{238} \mathrm{U}$ alpha exposures.

32 Result of applying a $100 \mathrm{~cm}^{2}$ convolution kernel to Figure 30 .

33 Result of applying a $100 \mathrm{~cm}^{2}$ convolution kernel to Figure 31 . 
volution kernel. Array was exposed to a clean surface in the laboratory for 1 hour.

35 Statistics, including histogram, for a background reading after applying a $35 \times 35$ convolution kernel. Array was exposed to a clean surface in the laboratory for 13 hours.

36 Result of a 177 minute array exposure to $7900 \mathrm{dpm}, 10 \mathrm{~cm} \times 15 \mathrm{~cm}\left(150 \mathrm{~cm}^{2}\right)^{241} \mathrm{Am}$ source.

37 Result of 64 minute and 6 minute array exposures to $7900 \mathrm{dpm}, 10 \mathrm{~cm} \times 15 \mathrm{~cm}(150$ $\left.\mathrm{cm}^{2}\right)^{241} \mathrm{Am}$ source.

38 Result of applying the calibration factor of 3.93 units per dis $/ 100 \mathrm{~cm}^{2}$ to Figure 37 . The six minute exposure is intended to approximate a 60 minute exposure to a 500 $\mathrm{dpm} / 100 \mathrm{~cm}^{2}$ source.

39 Result of a 150 minute array exposure to ${ }^{238} \mathrm{Pu} 660 \mathrm{~cm}^{2}$ source with an average activity distribution of $2000 \mathrm{dpm} / 100 \mathrm{~cm}^{2}$.

40 Result of applying the calibration factor of 4.02 units per dis $/ 100 \mathrm{~cm}^{2}$ to Figure 39 .

41 Result of a 150 minute array exposure to ${ }^{238} \mathrm{Pu} 660 \mathrm{~cm}^{2}$ source with an average activity distribution of $200 \mathrm{dpm} / 100 \mathrm{~cm}^{2}$. This source has one-tenth the activity of that used in Figure 39. Although there is no apparent source outline shown here, see Fig. 42.

42 Result of applying a $35 \times 35$ convolution kernel and calibration factor to Figure 41 .

43 Statistics, including histogram, for Figure 42. Compare to the background reading statistics in Figure 35.

44 Result of exposing an array to three $100 \mathrm{~cm}^{2}$ beta or beta/gamma sources. All three exposures were 208 minutes duration.

45 Result of applying a $35 \times 35$ convolution kernel to Figure 44 .

46 Statistics for pixel response to an approximately uniform beam, ${ }^{137} \mathrm{Cs}$ gamma exposure. The approximate exposure was $47 \mathrm{mR}$.

47 Result of an array reading given a combination exposure to ${ }^{238} \mathrm{U}$ alphas and $20.7 \mathrm{mR}$ ${ }^{137} \mathrm{Cs}$ gammas. Note that each of the three regions received a total exposure time of 30 minutes to ${ }^{238} \mathrm{U}$.

48 Result of applying a $35 \times 35$ (approximately $100 \mathrm{~cm}^{2}$ ) convolution kernel to Figure 47 . Note that, although visible in Figure 47 , the alpha exposures are essentially undetected in this figure.

49 Result of applying a $5 \times 5$ convolution kernel to Figure 47 . Compare to Figure 48 and note that the alpha exposures are visible when using the smaller convolution kernel. 
50 Simulation of background subtraction and signal processing on Figure 47. This figure simulates background subtraction by subtracting a constant value slightly less than the peak values obtained from the four 7.5 minute exposures and then setting any negative values to zero. The "background corrected" result was convoluted with a $33 \times 33$ kernel to obtain the above result. 



\section{Foreword}

The Nuclear Regulatory Commission utilizes the Small Business Innovative Research (SBIR) program, to fund small scale research projects that may serve to enhance public health and safety and promote the development of small businesses. These projects are selected based upon the agency's determination that the research can eventually result in an independent and self-sustaining business and application that will benefit the public safety and the NRC's prime directive to assure the same.

This report discusses the research results of a two phase SBIR project on the use of a two-dimensional laser heated thermoluminescense dosimetry system for surveying for residual radioactive contamination. The research presented in this report describes the Phase I effort to explore the feasibility of applying an existing two-dimensional radiation level mapping system, developed by the contractor, to detect, locate, and measure residual radioactive contamination and the progress made in Phase II to improve and modify the system to tailor it to this particular application. The potential advantages of the system over standard techniques include: the ability to detect contamination in otherwise inaccessible areas and greater reliability as a result of eliminating uncertainties inherent in the hand-held instrument scanning process.

The NRC staff believes that the methodology explored in this study may prove useful for residual radioactivity surveying when more fully developed. NUREG/CR-6037 is not a substitute for NRC regulations, and use of the methodology described herein is not required. The approaches and/or methods described in this NUREG are provided for information only. Publication of this report does not necessarily constitute NRC approval or agreement with the information contained herein.

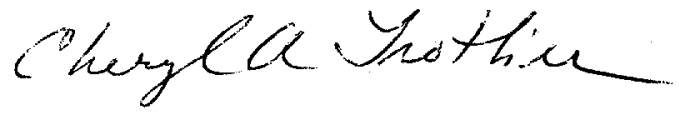

Cheryl A. Trottier, Acting Chief

Radiation Protection and Health

Effects Branch

Division of Regulatory Applications

Office of Nuclear Regulatory Research 


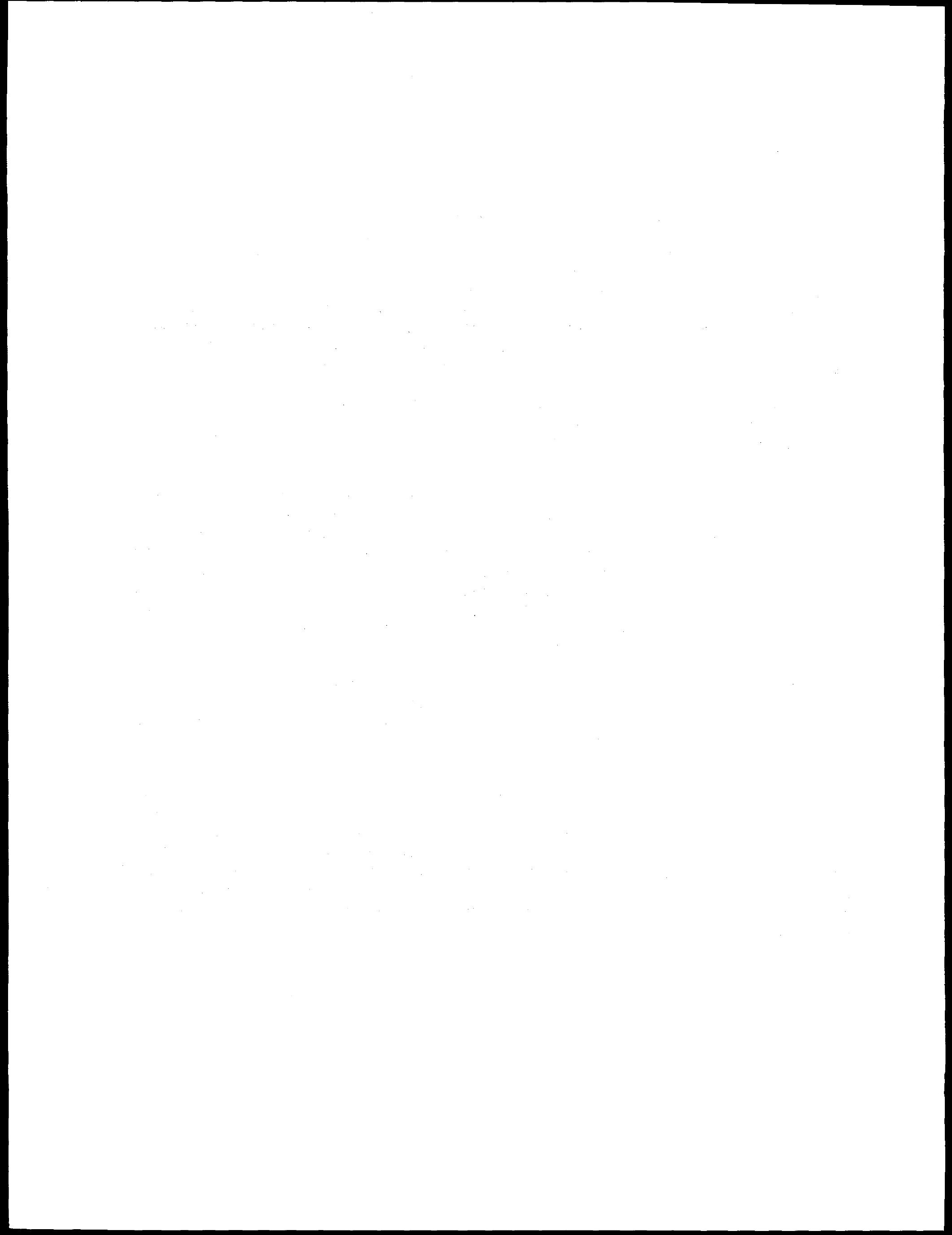




\section{PREFACE}

This document serves as the final technical report for a project that was carried out in two phases under the Small Business Innovation Research (SBIR) program funded by the US Nuclear Regulatory Commission. The two phases were separately funded, and a Phase I final report was prepared in 1993 but not published. The present document therefore contains two principal parts, the first part consisting of the Phase I final report, essentially unaltered, and the second part is the final report for the Phase II effort. In the intervening period between the preparation of the Phase I report and the completion of the Phase II effort, many developments in survey methodology and the regulatory environment have occurred. Some effort has been expended in updating the initial report to the current state of affairs, but it nevertheless is intended to reflect the contractor's level of knowledge at that time.

Both the SBIR Phase I and II efforts were originally contracted with International Sensor Technology, Inc., a small business in Washington State. During the course of the Phase II contract, International Sensor Technology was acquired by Keithley Instruments, Cleveland, Ohio, and the Phase II contract was assigned to Keithley.

The Phase I effort covers a feasibility study concerned with the possible application of a laserheated thermoluminescence dosimetry (TLD) system, developed for two-dimensional radiation dosemapping, to the measurement of low-level radioactive surface contamination. The results of the Phase I project were sufficiently promising to warrant additional work under a Phase II contract. This second project was primarily aimed at technically improving and modifying this system to tailor it to this specific application. The focus was on improving the system detection capability for alpha and beta radiation, and translating the output of the measurement system into a form useful in reporting surface contamination values. Experts were consulted for obtaining information on field requirements and general applicability of the system to surface contamination measurements.

The Phase II project was beset by problems both technical and nontechnical in nature. A wholly unexpected and unexplained phenomenon involving the component materials of the two-dimensional TLD arrays arose, which threatened to eliminate the possibility of applying the system to low-level contamination measurement. Over the originally estimated two year effort, solving this problem consumed all or part of eleven months, and is not yet completely resolved.

Under the proposed Phase II effort, a number of field tests and other collaborative efforts were to be performed. During the conduct of this project, however, significant reorganization of DOE programs occurred, with concomitant downsizing, resulting in the inability to follow through on these plans. Finally, the acquisition by Keithley of International Sensor Technology, and the subsequent relocation to Ohio, resulted in the loss of two of the three project staff personnel before project completion. Therefore, not all of the originally planned objectives were met, but significant progress was achieved. The work reported here indicates that laser-heated TLD constitutes a new capability in the area of surface contamination measurement. 


\section{ACKNOWLEDGEMENTS}

The author wishes to acknowledge the major contributions of two former employees of International Sensor Technology to this work. Jerry Sweet, enduring much trial and error, implemented virtually all of the electronic circuitry and software changes required to achieve the level of improvement in the Laser TLD system necessary to measure low levels of surface contamination. Gary McConnell was given the daunting task of finding a workable solution to the problem of the array phosphor binder. His enthusiasm and determination in doing so allowed the continuation of the project to the result reported here. Both also contributed in many other ways. Peter Braunlich, president of International Sensor Technology, provided the inspiration to propose and undertake this project, and his enthusiasm for developing widespread application of Laser TLD allowed it to take place.

Pete Hulbert took over many tasks and assisted greatly in completing the project after our move to Keithley Instruments. 


\section{INTRODUCTION}

This report presents the results of a two-phase study of the applicability of a laser-based thermoluminescent dosimetry system to the problem of measuring low-level radioactive contamination of surfaces by alpha and beta emitting nuclides. The study was funded under the Small Business Innovation Research (SBIR) program. Its first goal was to determine the feasibility of using an existing twodimensional radiation dose mapping system, developed by the contractor, to effectively detect, locate and accurately measure residual radioactive contamination. Such information is essential to determine whether an object must be cleaned, or to certify the safety of potentially contaminated materials for release from contamination areas. To this end, the first phase of the study served as a basis for proposing further development of the capability under an SBIR Phase II program. In Section 2 of this report, we present the findings of the Phase I feasibility study on the ability of this system, in its then current state of development, to measure localized, low-level alpha and beta particle emission. Section 3 of the report contains details on the improvements and newly developed features that were specifically oriented towards optimizing the system for detection of surface contamination undertaken in Phase II.

The basis of the investigation is a system consisting of a laser-based reader and monolithic arrays of thermoluminescent dosimeters (TLDs). The system was originally developed for dose field mapping of radiation therapy accelerator beams, and has found use in similar applications, such as mapping of flash $X$-ray sources used in radiation hardness testing of electronic components and systems. The arrays consist of nominally 40 micron thick TLDs spaced on a $3 \mathrm{~mm} \times 3 \mathrm{~mm}$ grid, covering an area of $30 \mathrm{~cm} \times 30 \mathrm{~cm}$, for a total of $900 \mathrm{~cm}^{2}\left(1 \mathrm{ft}^{2}\right)$. The reader heats each of the $10,201 \mathrm{TLD}$ sequentially with a $\mathrm{CO}_{2}$ laser, requiring about one hour to evaluate an entire array, and records the radiation dose values as a function of position, outputting the result as an isodose contour map. The arrays are fabricated on 125 micron thick, flexible polyimide substrates, which easily conform to regular surface shapes. The thickness of the array elements is about equivalent to the range of a typical alpha particle emitted by common radioactive materials, which is ideal for detection of these short range particles.

The general problem addressed here is surveying for radioactive contamination in sites, facilities and materials, with the eventual goal of cleaning and/or releasing the surveyed surfaces for reuse, thereby reducing the amount of material classified as nuclear waste. In particular, contamination by and removal of low-activity radioactive matter emitting low energy $\beta$ (beta) and weakly penetrating $\alpha$ (alpha) particles is important for facility or material release. The presence of these emitters pose a risk for subsequent occupation or re-use of facilities or materials by humans. The problem with surveying sites or material for the presence of low-level, short range radiation is one of sensitivity, measurement time and physical access. What is required, in the case of very low activity, is an integrating detector, yet one that is thin enough to measure short range radiation. These requirements are the most difficult to meet with hand-held devices used to sweep an area. A hand-held $\alpha$ monitor held stationary over a contaminated area having an activity level of 500 disintegrations per minute (dpm) per $100 \mathrm{~cm}^{2}$ will average about two counts per second. In addition, a "thick" detector (the detector itself being thick or having thick packaging, coatings or windows) is unsuitable for measuring short range particles: alpha particles of $5 \mathrm{MeV}$ energy have a penetration of only $3.8 \mathrm{mg} / \mathrm{cm}^{2}$ (a thickness of $38 \mu \mathrm{m}$ in a material of $1 \mathrm{~g} / \mathrm{cm}^{3}$ density). A practical lower detection limit may be as high as thousands of disintegrations per minute per $100 \mathrm{~cm}^{2}$ for sweep surveys (Martin). One dated recommendation was to set the lowest practical limit on beta emitting hot particles (considered as a point source relative to $100 \mathrm{~cm}^{2}$ ) at $5000 \mathrm{dpm}$ for sweep surveys (Sommers, 1975). The integration issue arises from signal to noise ratio considerations: the instrument must be able to accumulate sufficient information to overcome noise and background effects. A real-time, hand held detector may not be capable of detecting low activity levels. Further, the geometry of many objects prevents survey with standard instrumentation altogether, which may be falsely classified as contaminated even if the objects are clean. A thin, flexible and sensitive integrating detector is a necessary component in achieving comprehensive site 
surveys, allowing close proximity coverage to virtually every square inch of outer or inner surface area of a decommissioned structure or object.

The totally passive detector element array can be placed in contact with walls, inside and outside pipes or instruments such as computers or laboratory equipment, in short, surfaces that cannot be surveyed by standard instrument scan methodology. The array can be left in place for extended periods of time, integrating the dose delivered to the thin TLD elements by areas of low-activity surface contamination. The sensitivity of an individual element is adequate to detect 1-2 $\alpha$ particles absorbed. The thinness of the TLD array is ideal for monitoring short-range radiation as the background signal from $\gamma$-radiation is minimized by this configuration. The areal measurement density of the array provides for localization of contamination "hot spots," and provides ample background radiation information.

The Multi-Agency Radiation Survey and Site Investigation Manual (MARSSIM) (NUREG-1575) provides draft guidance on conducting radiological surveys for decontamination and decommissioning activities. It contains a valuable compendium of radiological survey methodology and includes a discussion of the measurement sensitivities of the various types of active and passive $\alpha$-and $\beta$-detectors. In particular, minimum detectable surface contamination figures for scanning and static survey methodologies are compiled. During the course of the present study, it was widely believed that contamination levels at the release guideline limits (see Table I in Sec. 2.2.1) were undetectable using active detectors, especially for the case of transuranic $\alpha$-emitters. While this is generally true for scanning surveys, static measurements using active detectors are capable of detecting alpha emission at release guideline levels. However, this is a very tedious process. The Laser TLD approach, as are other passive (i.e., integrating) techniques, is sensitive to arbitrarily low activity levels, in principle; there is a minimum integrating-time period required to achieve detection of a given level of activity. Among the two passive alpha detection methods included in MARSSIM, electret ion chambers and track detectors, the Laser TLD technique compares very favorably. The exposure or deployment times required are comparable, yet the TLD detectors, are flexible and can be cut into shapes, like track detectors, and cover up to $900 \mathrm{~cm}^{2}$ areas, in comparison to track detectors at $2 \mathrm{~cm}^{2}$ and rigid electrets at about $45 \mathrm{~cm}^{2}$.

This overall study was performed as two separate projects. Therefore, the two phases of the study are discussed in the sequence of the work performed. The feasibility component, Phase I, is reported in Section 2. Here, the capabilities of the system at the then current state of development are covered. In Section 3, the Phase II improvements and the now current capabilities are discussed 


\section{PHASE I FEASIBILITY STUDY}

The Phase I project was structured around seven tasks designed to demonstrate the feasibility of the system for this application. These seven tasks were: (1) Identification of alpha and beta emitting nuclides of concern and the relevant activity levels, concentrating on alpha emission; (2) Determination of the sensitivity of the system to appropriate activity levels (in the system's then current state of development), thereby deriving array exposure times required to achieve the measurement of the relevant activities, for both isolated contamination patches and uniformly dispersed activity. A study of nonradiation induced background signal was also performed during this task; (3) Determination of the array readout resolution necessary to achieve thorough survey coverage. This is important because array evaluation speed is a function of readout resolution; (4) Study the effect of homogeneous background radiation on the detectivity of the system; (5) Study array packaging in terms of reduction in sensitivity and prevention of light induced thermoluminescence signal; (6) Estimate the economics of eventual system deployment; (7) Analyze areas of improvement to the system to optimize its performance for the envisioned application. These project tasks are detailed below. First, a description of the system as used in the feasibility study is presented. Improvements developed during the Phase II effort are detailed in Section 3.2.

\subsection{System Description}

Laser-heated thermoluminescence dosimetry (LHTLD) provides enhanced sensitivity over conventional heating of TLDs because the fast heating rates (up to $1000^{\circ} \mathrm{C} / \mathrm{sec}$ ) extract the dosimetric information (the TL glow curve) in fractions of a second compared with the 30 seconds typical of conventional systems, greatly improving the signal-to-noise ratio when using standard luminescence detection components (photomultiplier tube and integrating electronics) (Braunlich, 1981; Braunlich 1990; Bloomsburg, 1990; Jones, 1992; Jones, 1993). This, in turn, allows for the use of much smaller TLD phosphor masses and therefore thinner dosimeter configurations to achieve the same lower limit of radiation dose detection, while increasing processing speed by up to a factor of 100 . Among other advantages, low mass, thin dosimeters are the optimum configuration for measurement of short range radiation.

In this system, a $\mathrm{CO}_{2}$ laser rapidly heats small TLD elements ("spots") evenly spaced in a monolithic array on a $3 \mathrm{~mm} \times 3 \mathrm{~mm}$ grid. The standard array covers an area of $300 \mathrm{~mm}$ square $\left(1 \mathrm{ft}^{2}\right)$ and contains 10,201 elements deposited on a polyimide substrate. About 60 minutes is required to evaluate an array, including isodose contour computation. The current system can accommodate arrays up $30 \mathrm{~cm} x$ $30 \mathrm{~cm}$. The arrays are indefinitely reusable with periodic calibration.

The optical system is depicted schematically in Fig. 1 . The $\left(10.6 \mu \mathrm{m}\right.$ wavelength) $\mathrm{CO}_{2}$ laser power is regulated by closed-loop control. A beam sample is compared to a programmed dosimeter heating cycle and corrections are made at $2 \mathrm{msec}$ intervals. The laser beam is modified by an optical channel which produces a square beam profile of uniform intensity. The imaging lens relays the channel output to the plane of the array, where the image is a $2 \mathrm{~mm} \times 2 \mathrm{~mm}$ square, overlapping the $1.5 \mathrm{~mm}$ diameter circular elements. The channel and imaging lens are mounted in a scanning optical head. A flexible fiber optic bundle picks up the emitted TL and conducts it to a cooled photomultiplier tube. A moving, dimpled vacuum platen holds the array down in a reproducible position. By a combination of moving the optical head and platen, each dosimeter element is read out sequentially. The heating time per element is dependent on phosphor type, varying from about 180 to $350 \mathrm{msec}$. The dimples retard heat flow into the platen, and the dimple walls provide thermal isolation between dosimeters. All functions are controlled by embedded circuitry, while a personal computer (PC) provides disk storage and graphic display. The system is capable of readout at various resolutions on a single sheet, to speed up evaluation; $6 \mathrm{~mm}$ resolution 


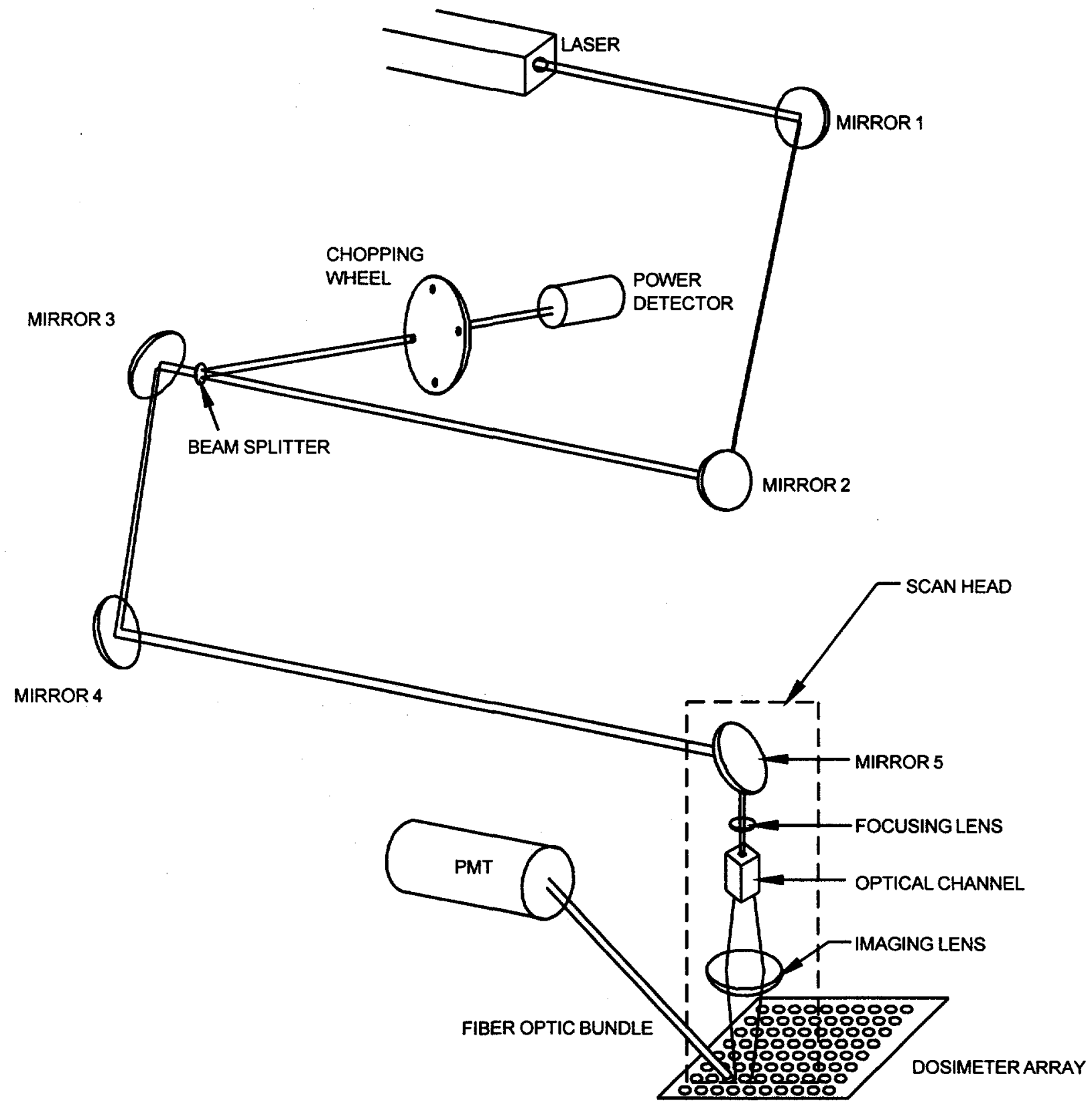

Fig. 1: $\quad$ Schematic of the laser TLD reader optical system. The laser is controlled by feedback generated from the split-off beam sample. The optical channel transforms the Gaussian beam into a square of uniform profile. The fiber optic bundle conducts the emitted thermoluminescence to the photomultiplier (PMT) for intensity measurement. 
results in array readout time of 15 minutes, and at $12 \mathrm{~mm}$ resolution, the readout cycle requires about four minutes. Various analytic functions are preprogrammed in the reader system.

The dosimeter array elements, or "spots," consist of phosphor powder (20 - $40 \mu \mathrm{m}$ grain size) mixed with a high temperature silicone adhesive. Each is $1.5 \mathrm{~mm}$ in diameter, $40 \mu \mathrm{m}$ thick and has a mass of about $90 \mu \mathrm{g}$. The substrates are $125 \mu \mathrm{m}$ thick aluminized polyimide film. The $100 \mathrm{~nm}$ thick sputtered aluminum layer reflects laser energy not absorbed by the dosimeters (rather than being absorbed by the substrate) and also reflects the emitted TL radiation into the light collection optics. Fig. 2 depicts the fabrication of an array.

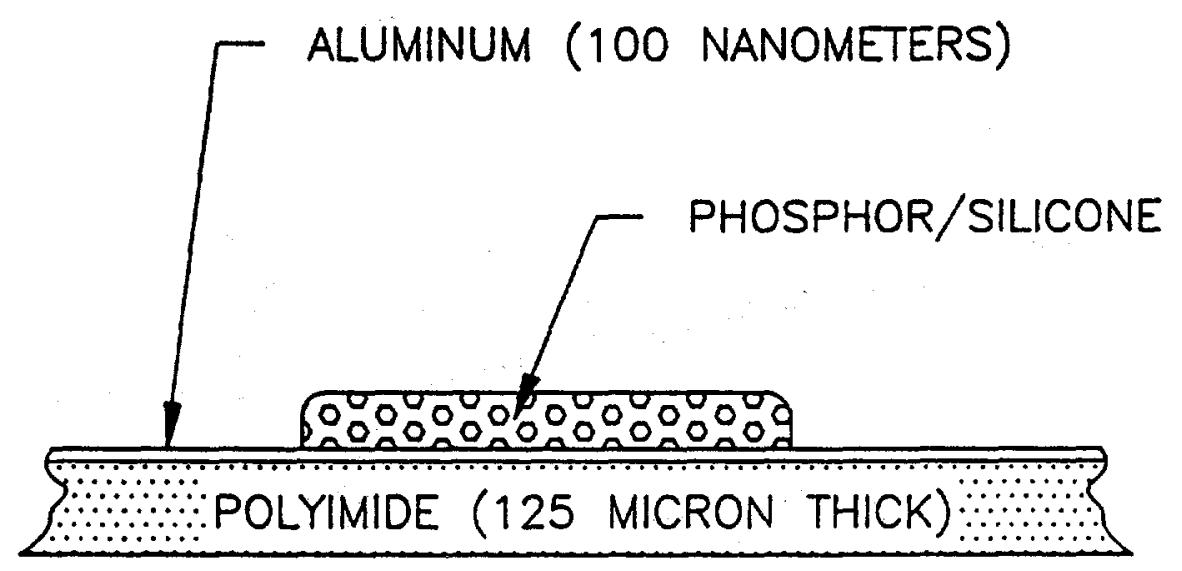

Fig. 2: Cross-sectional view of TLD array construction.

\subsection{Findings From Phase I Effort}

The results obtained in the seven task areas of the Phase I project are presented in this section. The primary goal of the Phase I effort was to establish the feasibility of applying the LHTLD array system to the problem of detecting and locating residual radioactive contamination for facility decontamination and decommissioning activities, and general surveys for material release. 


\subsubsection{Applications}

The first task was to determine the appropriate application of this technology based on researching the specific needs of those faced with decontamination responsibilities. Interviews with various experts have revealed a much wider range of applications than anticipated in the Phase I proposal, and show that the LHTLD array system has unique potential for providing contamination survey measurements that are currently impossible, or very time consuming and expensive. The problem of material release (i.e., certifying that an object is free of radioactive contamination for removal from a radiologically controlled area) is one where standard methodologies, a scan survey with alpha and beta/gamma monitors ${ }^{a}$ is impossible in cases where the survey instrument is too large to be brought into the required proximity with the surfaces of the object. For instance, it is not possible to insert the appropriate monitor into pipes smaller than a few inches in diameter, yet if such objects are deemed to be potentially contaminated, they must be assumed to be unless shown otherwise. The TLD arrays can be applied to this example, providing the requisite survey, and thereby allowing for the uncontrolled release of such items, reducing the burden on radioactive waste facilities and letting such items be reused, another cost savings. Other problems arise from simple geometry. A scanning monitor may not be able to achieve the necessary $6 \mathrm{~mm}$ proximity to a surface in the corner of a cabinet, or inside a computer that has been in a potential contamination area. The arrays provide a very simple solution, because they are thin and flexible. Thus, the arrays can provide contamination surveys in situations where it is not currently possible.

Other such instances are in contamination zones not associated with buildings. The arrays can be used (and have been tested) to measure alpha emitting ${ }^{239} \mathrm{Pu}$ contamination depth-profiles in soils. Such sites must currently be tested by soil layer removal $(1 \mathrm{~cm}$ thick layers from a 1 square meter area) and subsequent costly and time-consuming chemical analysis. Although the arrays cannot differentiate radionuclides, they can relatively easily detect the presence of radionuclides at depths up to $30 \mathrm{~cm}$ below the surface. Surveying large areas requires many TLD tests, but may cost less than one-tenth of the $\$ 10,000$ cost for a chemical test. ${ }^{\text {b }}$

In addition to these specific applications, the general problem of surveying even large, planar surfaces is not being satisfactorily met with current methodologies. In general, surveys of such surfaces are done by a technician scanning the surface by moving the monitor across the surface at a rate not to exceed $5 \mathrm{~cm} / \mathrm{sec}$ at a distance of $6 \mathrm{~mm}$ or less from the surface. ${ }^{\circ}$ The human factor in this method has been recognized to be problematic, in that it may take several hours to scan a laboratory door, and in many instances, the survey must be performed twice, once for alpha particle emission, and a second time for beta/gamma emission. A tendency to exceed the specified scan rate is understandable. In addition to this problem, survey instruments are not capable (in scanning mode) of detecting contamination at the desired levels for material release for some of the listed radionuclides (see Table I below) even under ideal conditions, and measurable activity may be 3 to 5 times the desired specific activity level for release. ${ }^{d}$ Such problems have led some to propose raising release activity guidelines for reasons of practicality (Sommers, 1975).

\footnotetext{
a James Durham, Battelle Pacific Northwest Laboratories, personal communication (1993).

'William Quam, EG\&G Energy Measurements, personal communication (1993).

`James Durham, Battelle Pacific Northwest Laboratories, personal communication (1993).

Ibid.
} 
We summarize here the present advantages of the LHTLD array system for material release surveys determined by our Phase I work:

- $\quad$ Survey of "inaccessible" surfaces is possible, with implications for waste reduction.

- Both alpha and beta/gamma emitters are detectable with a single survey.

- The TLDs are passive integrating dosimeters, therefore release activity levels do not have to be compromised because of survey instrument insensitivity, and results would not be subject to scan speed errors because the arrays are left in contact with the surface for a specified time period in order to accumulate sufficient dose for detection.

The following table summarizes the radionuclides of concern and the activity levels for uncontrolled material release (guidelines).

TABLE 1

ACCEPTABLE SURFACE CONTAMINATION LEVELS (US Atomic Energy Commission)

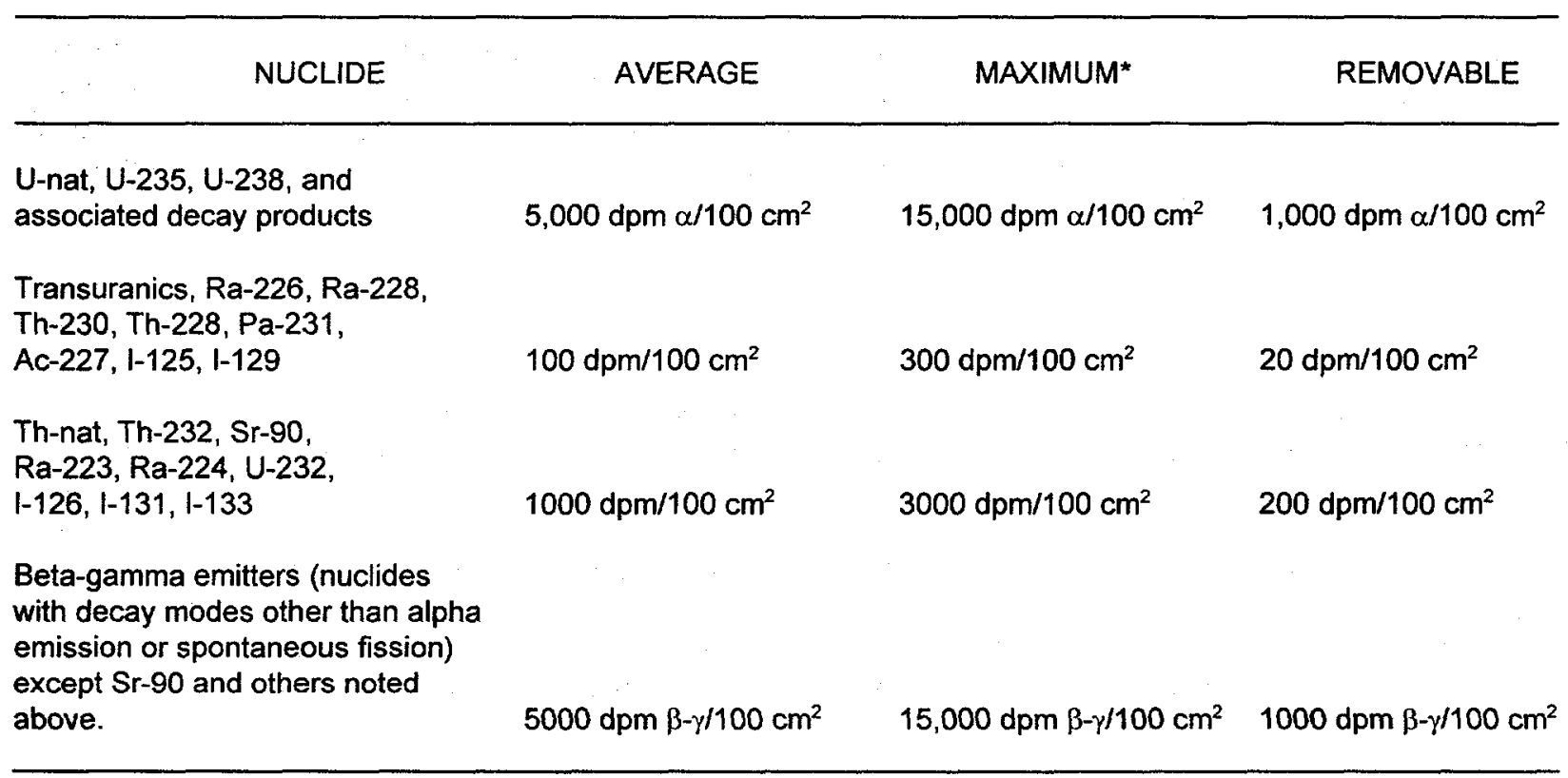

*The maximum contamination level applies to an area of not more than $100 \mathrm{~cm}^{2}$.

Table I. This table, reproduced from US Atomic Energy Commission Regulatory Guide 1.86 (1974), lists the NRC unrestricted release guideline values of maximum surface contamination by various radionuclides of concern for reactor decommissioning. 
Since the commencement of this work, the NRC has published a proposed rule (August 22, 1994; 59 FR 43200) and has just approved a final rule to establish radiological criteria for decommissioning. The final rule will likely be published in the same time frame as this report. The criteria include a dose limit for the release of lands and structures at decommissioning. Under the rule, the specific contamination limits for decommissioning in terms of $\mathrm{dpm} / 100 \mathrm{~cm}^{2}$, may vary considering site-specific factors. The rulemaking would not apply to the routine release of equipment and materials. Throughout this document, mention of release guideline limits refers to Table 1 of Regulatory Guide 1.86 (US Atomic Energy Commission, 1974).

\subsubsection{Sensitivity and Background Measurements}

The second task was to determine the array sensitivity and the requisite exposure time to achieve detection of the activity levels listed above. A series of experiments have shown that the system is generally sensitive to very low-level alpha exposures corresponding to the desired release levels, and will be able to serve in the capacity under study.

A variety of alpha emitting radiation sources was used to measure sensitivity in general, the background signal (which sets the lower limit of detection), and the spatial spread of radiation induced signal from approximate "point" sources of alpha emission. Two phosphor types were studied, $\mathrm{CaSO}_{4}$ doped with Dysprosium (Dy) or Thulium (Tm). For purposes of efficiency, $75 \mathrm{~mm}$ square arrays were fabricated for most measurements. This array size is more than adequate to accommodate commonly available alpha and beta emitting sources. Most exposures were performed with the source in direct contact with the array. In addition, a test was performed under severe field conditions with exposure of bare arrays to contaminated soil.

The test bed reader was modified to achieve higher sensitivity and lower noise midway through the feasibility phase of this project. The original configuration, designed for what would be considered high dose measurements relative to the present application (mapping of radiotherapy beams with doses generally exceeding $0.05 \mathrm{~Gy}$ ), was not optimized for these high sensitivity trials. The primary improvement was in increasing the collection of the thermoluminescence emission by approximately a factor of 15 . Other improvements during the course of the investigation make it impossible to directly compare all results. Thus, we concentrate on those results obtained with the final Phase I configuration of the reader system, but will also discuss those obtained during the reader improvement process. Most tests were performed with $\mathrm{CaSO}_{4}: \mathrm{Tm}$ phosphor because its use yields the least background from incandescence emission when the system photomultiplier is fitted with the appropriate color filter.

The principal radiation source for the alpha detection measurements was a disk shaped source of depleted ${ }^{238} \mathrm{U}$, with an activity of $466 \mathrm{dpm}$, distributed over an $8 \mathrm{~mm}$ diameter area, with a thin (100 micro$\mathrm{gram} / \mathrm{cm}^{2}$ ) acrylic cover. The average alpha particle energy is just below $4.2 \mathrm{MeV}$. This source closely approximates a "typical" contamination spot. The average area of one TLD array dot is $1.77 \mathrm{~mm}^{2}$, and thus this source, when in contact with an array, will impinge an array dot with 8 alpha-particles per minute (note that only half of the alphas can escape from the source, the other half going into the source backing). Figure 3 shows the resultant measurement of one such exposure of 30 minute duration, where the source was placed alone in the center of a small array. Figure 4 shows a TL glow curve from an alpha excited array element, and a second heating also. The signal used in sensitivity calculations is the area under the glow curve. In all array evaluations during Phase I, the result consists of the difference between a first and second reading. This strategy was adopted in order to achieve the maximum sensitivity with minimum background signal. Note that the array elements are not calibrated with respect to relative sensitivity, and over a large array, the extreme limits of sensitivity variation are about a factor of two. Thus, these results are presented as average response of array elements. 


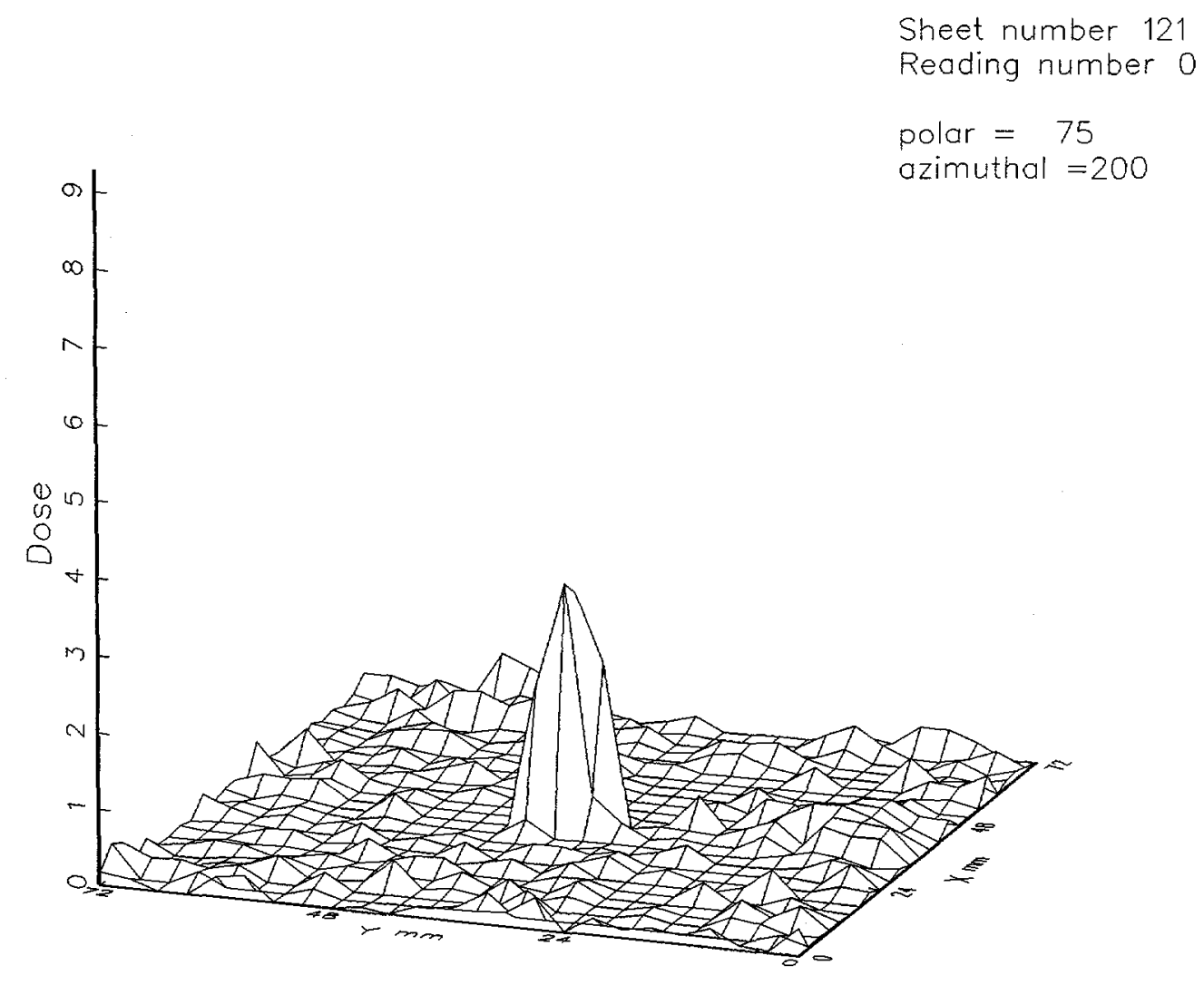

Fig. 3: $\quad$ Measurement result following a 30-minute exposure to the depleted ${ }^{238} \mathrm{U}$ alpha source. The activity is $466 \mathrm{dpm}$, over an $8 \mathrm{~mm}$ diameter area. The equivalent specific activity over the array area is $466 \mathrm{dpm} / 54 \mathrm{~cm}^{2}$. 

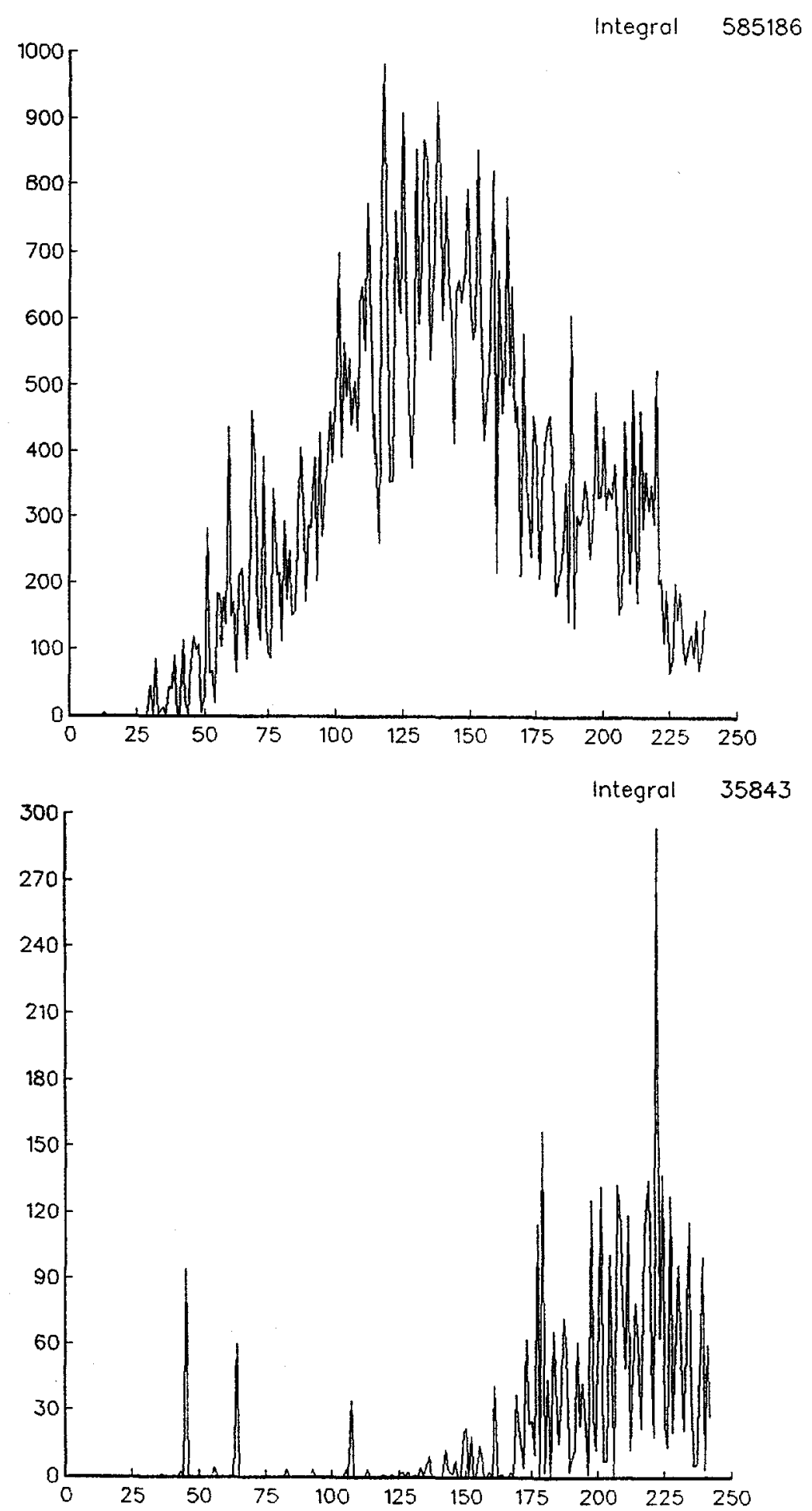

Fig. 4: $\quad$ Light output for first (above) and second (below) heatings of an alpha irradiated TLD. The difference between the two is the measure of signal. Note that significant signal begins at about $175 \mathrm{msec}$ in the second heating. 
A series of these measurements is plotted in Fig. 5 , for exposure durations lasting up to 900 minutes. In this figure, the average response of a TLD element is plotted against the average number of alpha particles incident on it. The result is that the response is proportional to exposure, as expected, but that the apparent background (the y-intercept) is very large, equivalent to about 230 alpha particles incident on a TLD. This clearly is not the case in general, as Fig. 3 shows the relative levels of signal and background for a 30 minute exposure to the same source, where about 240 alphas are incident on the array elements. This behavior is generally observed, where the background signal increases with exposure duration, but is not observed at this magnitude with unexposed arrays. In an attempt to decrease the background, the time interval for integration of the glow curve was shortened, from full length to $175 \mathrm{msec}$, where the second heating begins to register significant signal level (see Fig. 4), but maintained the full heating time. The results of this action are shown in Fig. 6 . Note that the signal level per incident alpha particle is maintained (compare the slopes of the fitted curves in Figs. 5 and 6), but in the second case, the apparent background ( $y$-intercept) is reduced by about a factor of seven, to approximately 33 alphas equivalent, when results from arrays having no exposure are included in the calculation.

Measurements of background signal are inconsistent, but generally fall into two groups. Background determinations of unexposed arrays (where the array was prepared by annealing or "resetting" them in an oven), or measurements in array regions far from the source, yielded either quite high, on the order of 30 alphas equivalent, or quite low values, equivalent to about 6 alpha particles incident on a TLD, after instituting the modified glow curve integration method. These results were obtained by fairly prompt reading of the background after annealing. Best performance examples of long term background signal accumulation was given by two arrays that were evaluated two months after annealing, where an average background signal equivalent to about 52 alpha particles incident on a TLD element was obtained. A third yielded 10 alpha equivalent background after 150 hours.

The majority of the background signal seems to arise in the silicone binder used to fabricate the arrays. A reading of an array consisting of silicone only, without phosphor, yielded a background signal equivalent to about 50 alphas per spot on average, but these elements contained about three times more silicone than in a normal phosphor spot. In the established applications of the TLD array system, this background problem is negligible. Given that low backgrounds were obtained from about $40 \%$ of these test arrays, we were confident that consistent, low backgrounds would be attainable in a Phase II effort. (This problem in fact became intractable, see Section 3.2 below) In addition, thinner elements (perhaps as thin as 25 microns or less) may improve the signal-to-background ratio. However, considering Fig. 3 , where a signal-to-average-background ratio of better than 30:1 is shown for 240 incident alphas per element, the potential for achieving detection of low-level contamination is demonstrated.

Some low-level image processing (spatial filtering) is available on the reader system, and Fig. 7 shows the results of a processed reading (all data discussed above is unprocessed) of an array given exposures to the ${ }^{238} \mathrm{U}$ source of $5,10,20$ and 40 minutes. The filtering in this case consists of an average of one element with its eight nearest neighbors with $\sin X / X$ weighting (equivalent to a sharp cut-off, low pass filter). Even though this array has a fairly high background (about 52 alphas per element), the five minute exposure (40 alphas per element) is visible.

Figure 8 shows the response of a $\mathrm{CaSO}_{4}: \mathrm{Tm}$ array to a $9000 \mathrm{dpm}, 25 \mathrm{~mm}$ diameter ${ }^{230} \mathrm{Th}$ alpha source (4.65 MeV average particle energy) for a four-hour exposure ( 3880 alphas per element average). Sensitivity at this energy for transuranics is similar to that of the ${ }^{238} \mathrm{U}$ alphas. This measurement was obtained before the reader was in its Phase I final configuration, however, and a slightly greater sensitivity to these alphas was expected, because they have greater energy to deposit in the phosphor per incident particle. Additional exposures conducted with a $0.5 \mathrm{~mm}$ diameter ${ }^{210} \mathrm{Po}$ needle source are discussed below in regard to the ability to detect small radioactive particles that may fall in between array elements. The sensitivity to these $5.4 \mathrm{MeV}$ alphas appears to be somewhat higher, again because of the higher particle energy, but the uncertainty in exposure (due to inverse-square dependence) is too large to make a firm conclusion on this point. 


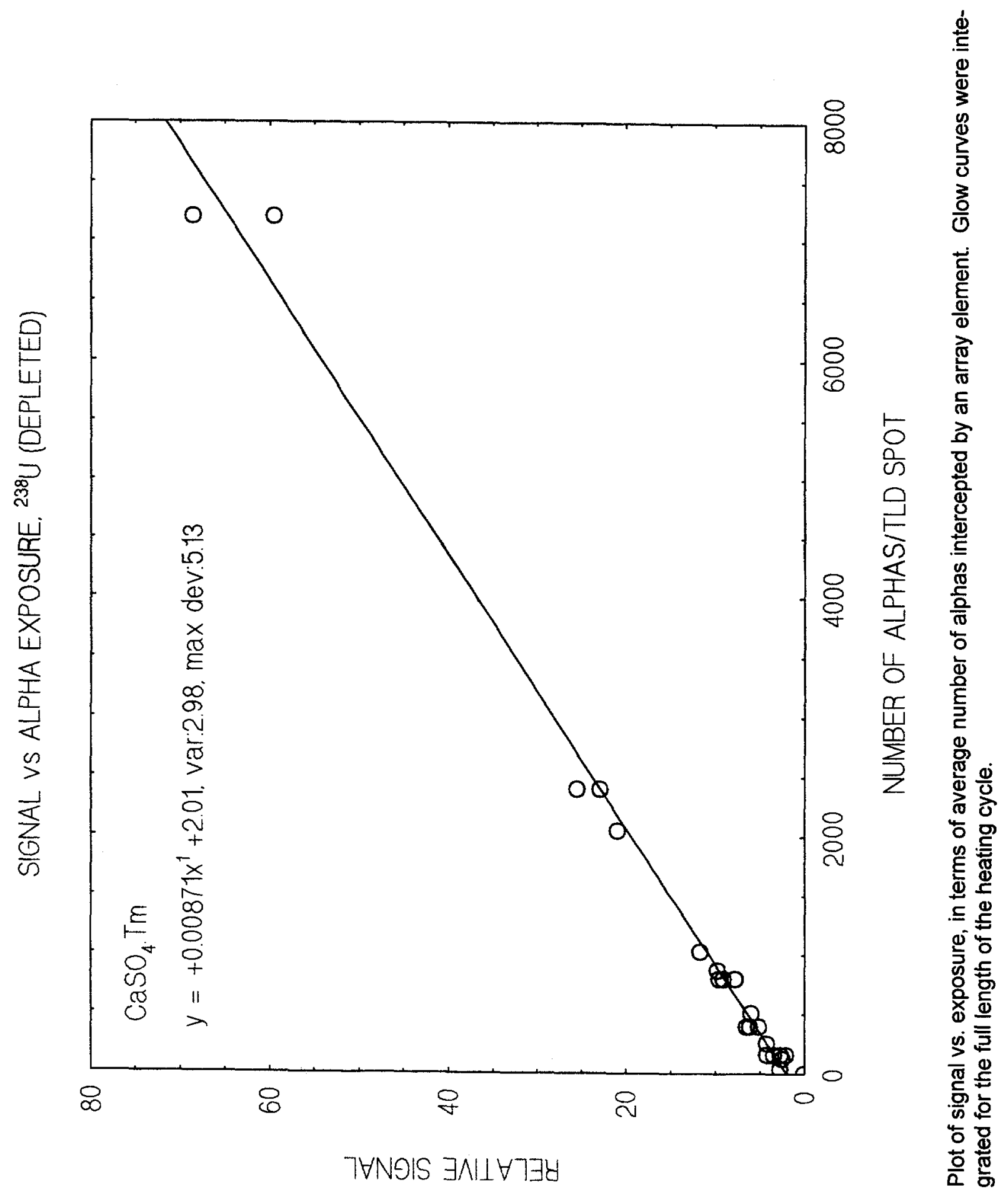

in 


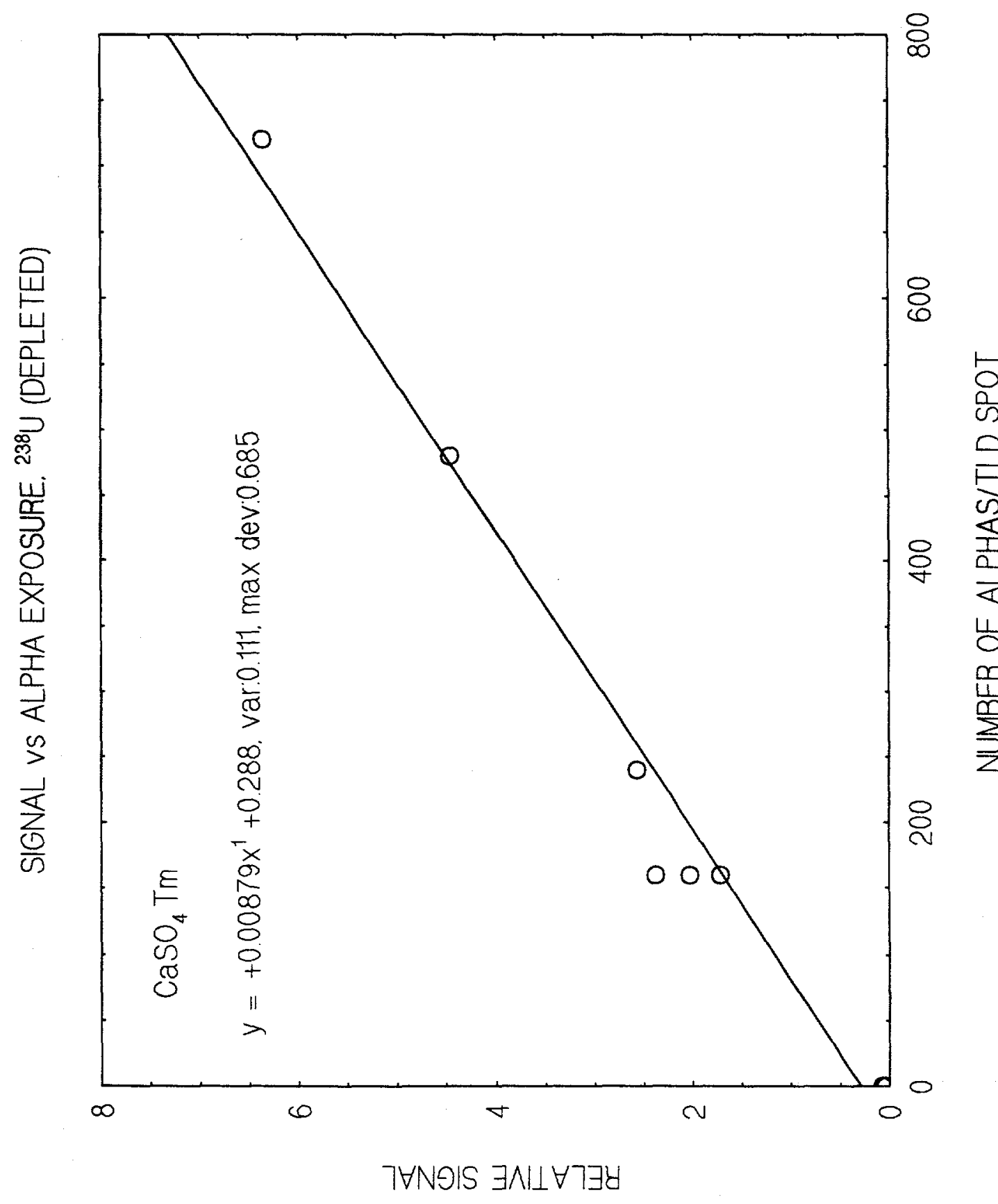

$\dot{0}$
$\dot{i}$ 

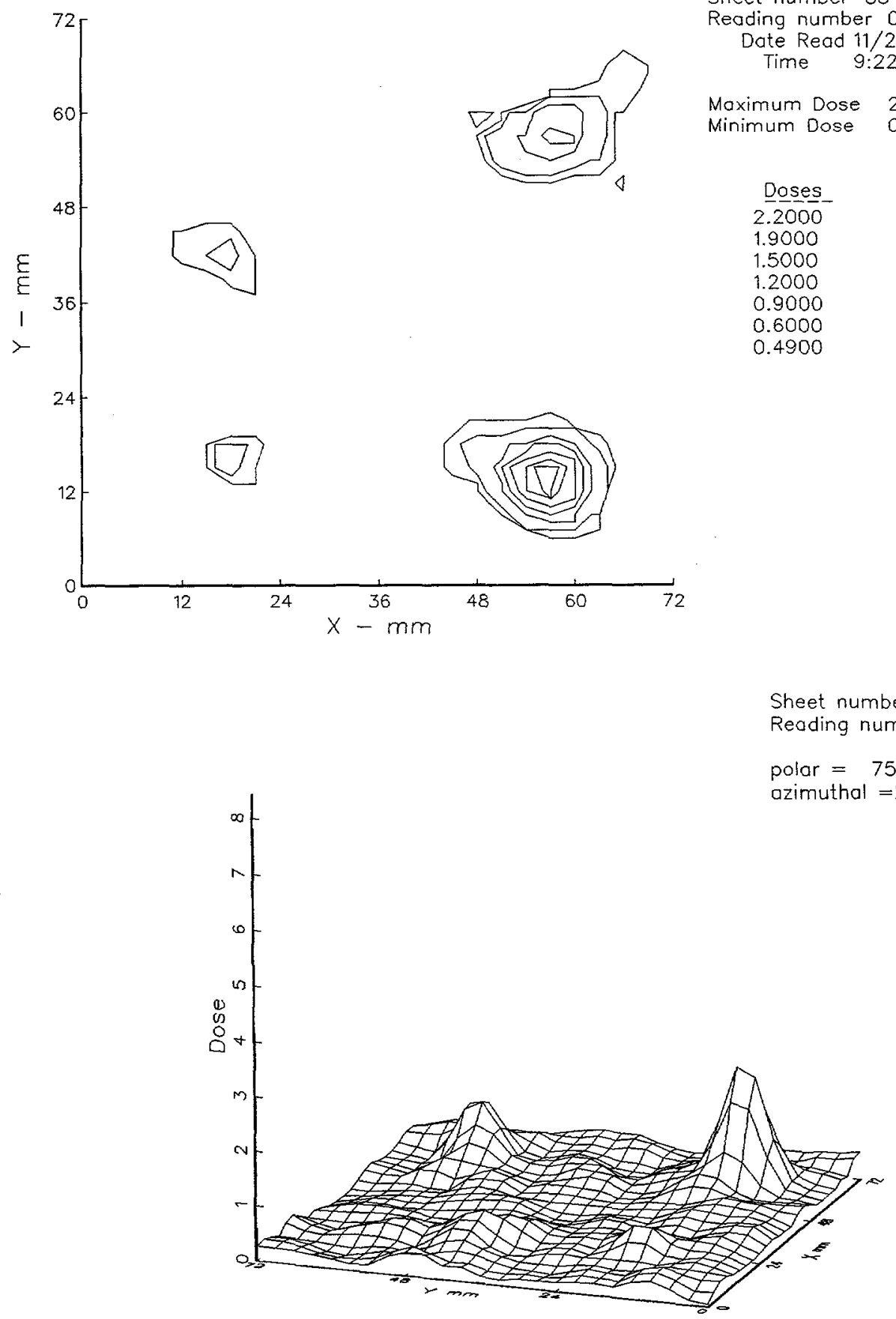

Fig. 7:

polar $=75$
Sheet number 85

Reading number 0

Date Read 11/2532650

Time $\quad 9: 22$

Maximum Dose 2.4325

Minimum Dose 0.0202

Doses.

2.2000

1.9000

1.5000

1.2000

0.9000

0.6000

0.4900

Sheet number 85

number 0

azimuthal $=200$

Resultant measurement following array exposure to $466 \mathrm{dpm}{ }^{238} \mathrm{U}$ alpha source after image processing (low pass filtering). Exposure duration referenced to the contour plot (above): 5 minutes - lower left, 10 minutes -upper left, 20 minutes upper right, 40 minutes - lower right. 
Sheet number 17

Reading number 0

polar $=75$

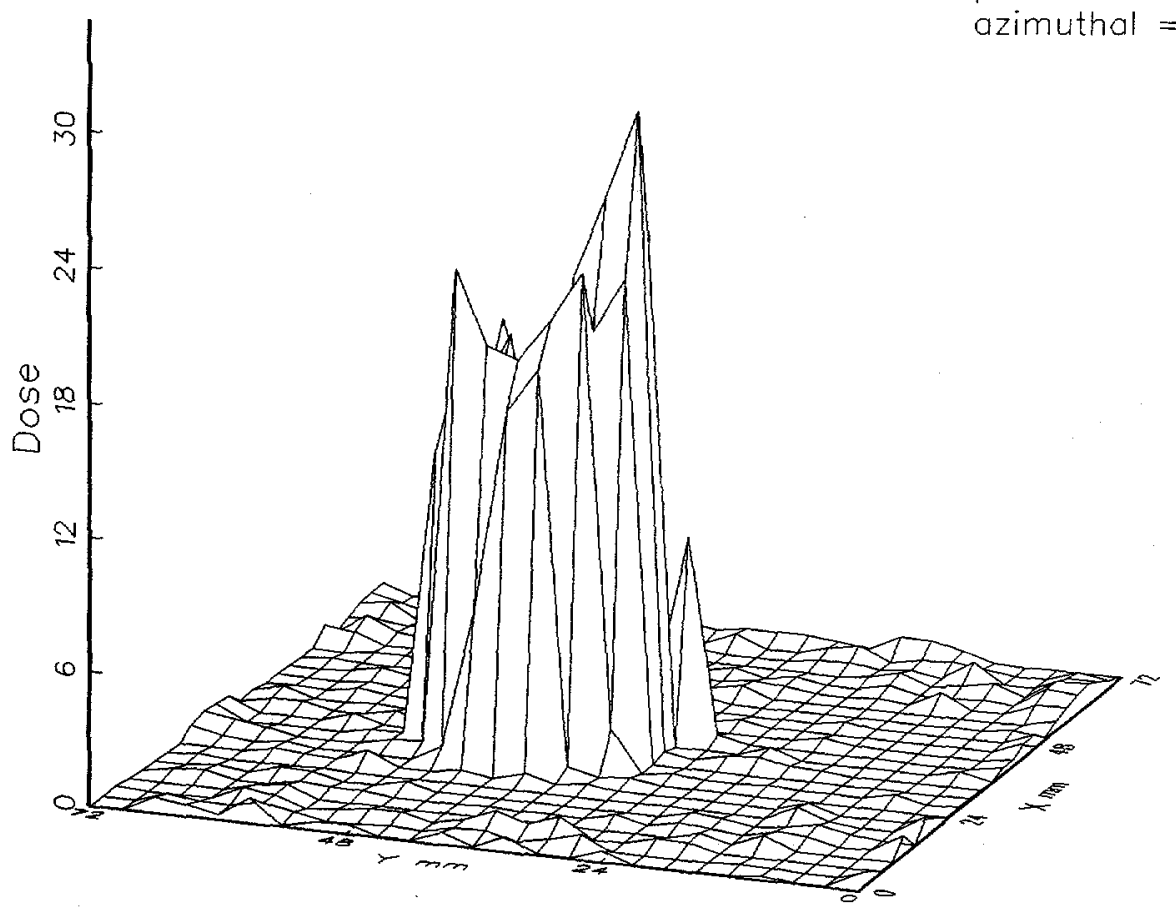

Fig. 8:

Array response to four hour exposure to $9000 \mathrm{dpm}, 25 \mathrm{~mm}$ diameter ${ }^{230} \mathrm{Th}$ alpha source. Array phosphor: $\mathrm{CaSO}_{4}: \mathrm{Tm}$. 
At the conclusion of Phase I, the exposure time required to accumulate a signal equal to the best background measurements for the alpha contamination limits listed in Table 1 are about 25 minutes for a uniform contamination of $5000 \mathrm{dpm} / 100 \mathrm{~cm}^{2}$, and about 21 hours for a uniform contamination of $100 \mathrm{dpm} / 100 \mathrm{~cm}^{2}$. Generally, contamination will occur in smaller areas (these limits are average values over scanned areas), and for instance, if the contaminant is concentrated in an area of about $4 \mathrm{~cm}^{2}$, these exposure times will be reduced by a factor of 25 . Additionally, improvements in background will also improve these exposure times. The background signal level discussed above (equivalent to 10 alpha particles per array element in 150 hours) represents a best case observed during Phase I. The equivalent activity level (for alpha emission) corresponds to only $10-15 \mathrm{dpm} / 100 \mathrm{~cm}^{2}$, which is well below the release guideline values. In Phase II, the developed improvements reduced the required exposure to about one hour for the detection of transuranic $\alpha$ emitters at release guideline limits $\left(100 \mathrm{dpm} / 100 \mathrm{~cm}^{2}\right.$.)

Exposures to beta sources were also studied in this task. Figure 9 shows the result of exposing a small array to six different weak beta sources simultaneously. The sources were all disks of approximately $1.25 \mathrm{~cm}^{2}$ area, with the following activity levels: ${ }^{147} \mathrm{Pm}$ (average energy $61.9 \mathrm{keV}$ ), $24,000 \mathrm{dpm} / \mathrm{cm}^{2} ;{ }^{36} \mathrm{Cl}(280 \mathrm{keV}), 33,000 \mathrm{dpm} / \mathrm{cm}^{2} ;{ }^{99} \mathrm{Tc}(84.7 \mathrm{keV}), 60,000 \mathrm{dpm} / \mathrm{cm}^{2} ;{ }^{210} \mathrm{Bi}(390 \mathrm{keV})$, $35,250 \mathrm{dpm} / \mathrm{cm}^{2} ;{ }^{14} \mathrm{C}(49.5 \mathrm{keV}), 290,000 \mathrm{dpm} / \mathrm{cm}^{2} ;{ }^{90} \mathrm{Sr}{ }^{90} \mathrm{Y}(949 \mathrm{keV}), 60,000 \mathrm{dpm} / \mathrm{cm}^{2}$. Each exposure was 60 minutes duration. The results generally scale with activity level, except for the lowest energy source. Array response per unit activity is plotted in Fig. 10 for these exposures. The response to ${ }^{14} \mathrm{C}$ was surprising, as detection was unexpected. However, it does demonstrate the threshold for low energy beta detection. The relative response to ${ }^{147} \mathrm{Pm}$ shows that the system is, however, capable of high sensitivity measurements of low energy beta emitters. Assuming the best case for background signal, the equivalent background for beta emission derived from the four highest energy sources in Fig. 10 is on average about 90 betas per TLD element, or 180 disintegrations. For a uniform contamination of 5000 $\mathrm{dpm} / 100 \mathrm{~cm}^{2}$ (the release guideline for most beta emitters), this works out to 210 minutes for a minimum exposure to achieve a signal equivalent to background ((signal + background)/background $=2$ ). For the lower limit on ${ }^{90} \mathrm{Sr}$, this figure would be of course five times greater, about 18 hours. Recall, however, that with this system exposure is passive.

One set of experiments under severe field conditions was performed. A series of six full-sized (101 x 101) TLD arrays of three different phosphor types were exposed uncovered (i.e., the TLD array was totally unprotected by any sort of cover) to a soil area known to be contaminated by ${ }^{239} \mathrm{Pu}$ at the $\mathrm{Ne}$ vada Test Site. This was a hostile environment for the TLD arrays, both because of the soil surface temperature and the possibility of contaminating the arrays with dirt particles, which could light up when hit by the reader's laser beam. Figure 11 shows the result of a test wherein a $\mathrm{CaSO}_{4}: \mathrm{Tm}$ array was exposed to the contaminated soil such that the right edge of the array (see right edge of plot in Fig. 11) was covered and could not have received alpha exposure. This example was from an array that was buried, rolled and inserted in the soil as a vertical tube for a total of forty minutes. The resultant indicated alpha emission (the spikes on the figure represent the locations of alpha emitting particles) correlate with the expected result based on the known contamination profile of the soil. At this exposure duration, the signal to noise ratio was about 10:1 at a calculated contamination concentration of about 35 picocuries per gram of soil. ${ }^{\theta}$ This result was aided by a manual glow curve discrimination procedure used to reject signals lacking the characteristic glow curve shape expected, as we anticipated severe contamination by dirt in this test. The technique appears to be valid and is described below. This feature was automated during Phase II. This demonstration of the utility of the system in contamination remediation applications points to potentially vast cost savings in location of radioactive particles, and is discussed in a later section.

${ }^{e}$ A. Bhattacharjie and W. Quam, in situ Measurement of Environmental Alpha Activity at Very High Spatial Resolution and Sensitivity, presented at the International Symposium of Field Screening Methods for Hazardous Wastes and Toxic Chemicals, Las Vegas, NV, Feb. 24-26, 1993. 


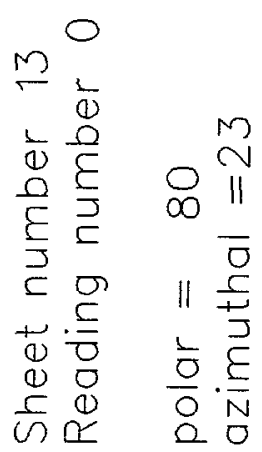

흥

商

0
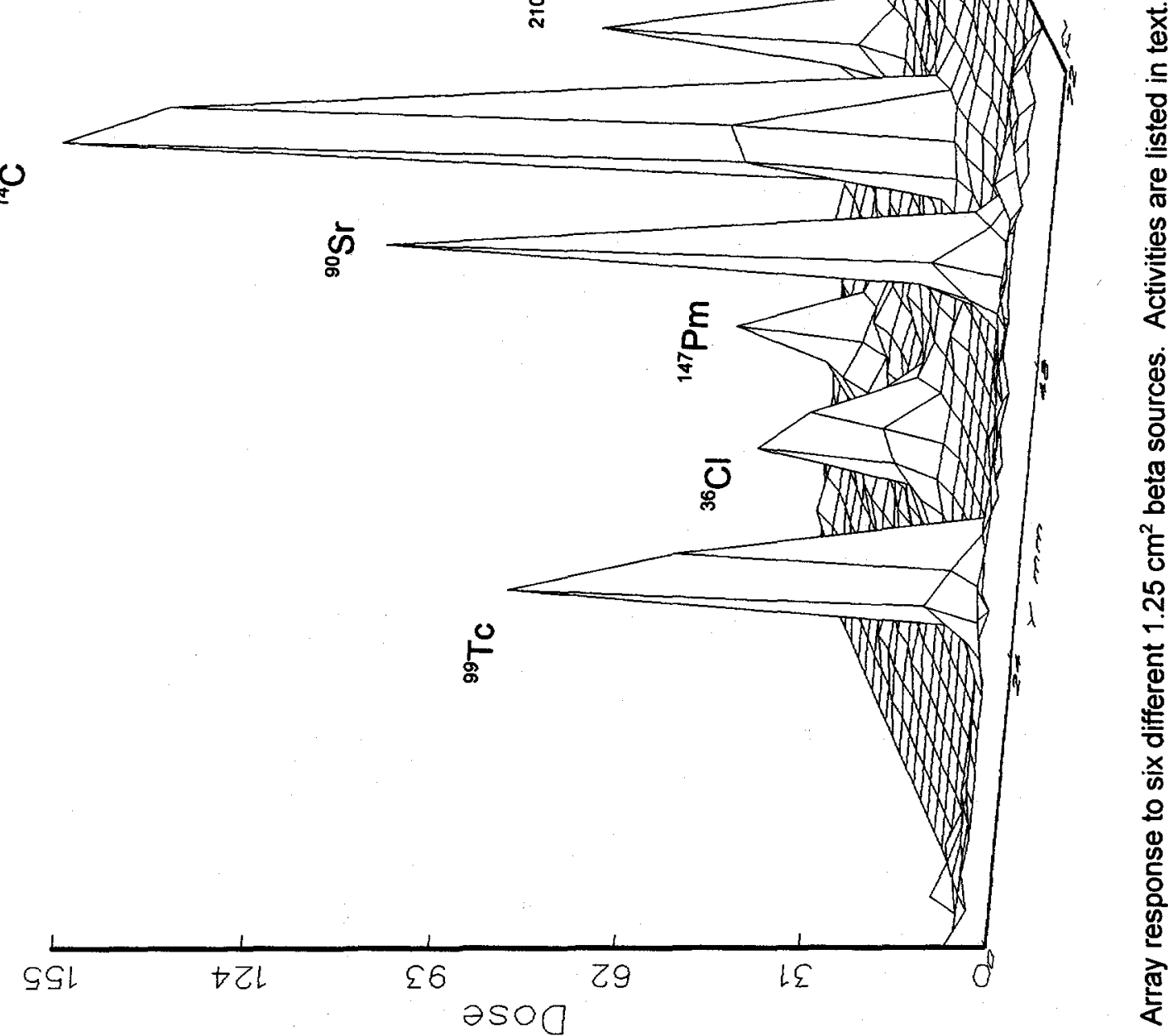

$\stackrel{\ddot{\circ}}{\dot{\Phi}}$ 


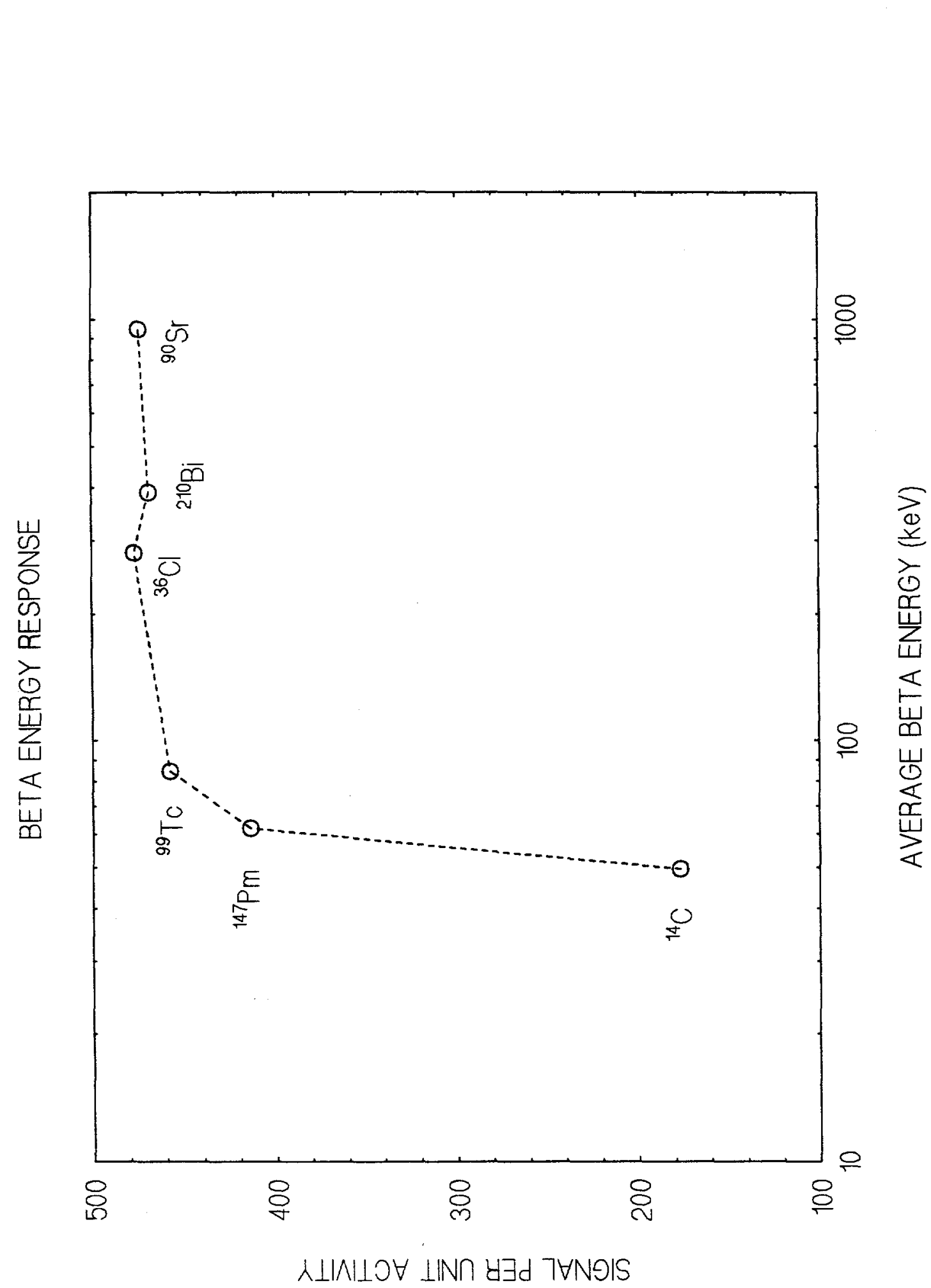

움 


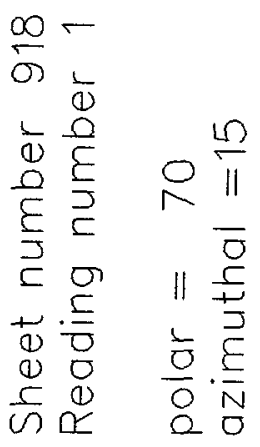

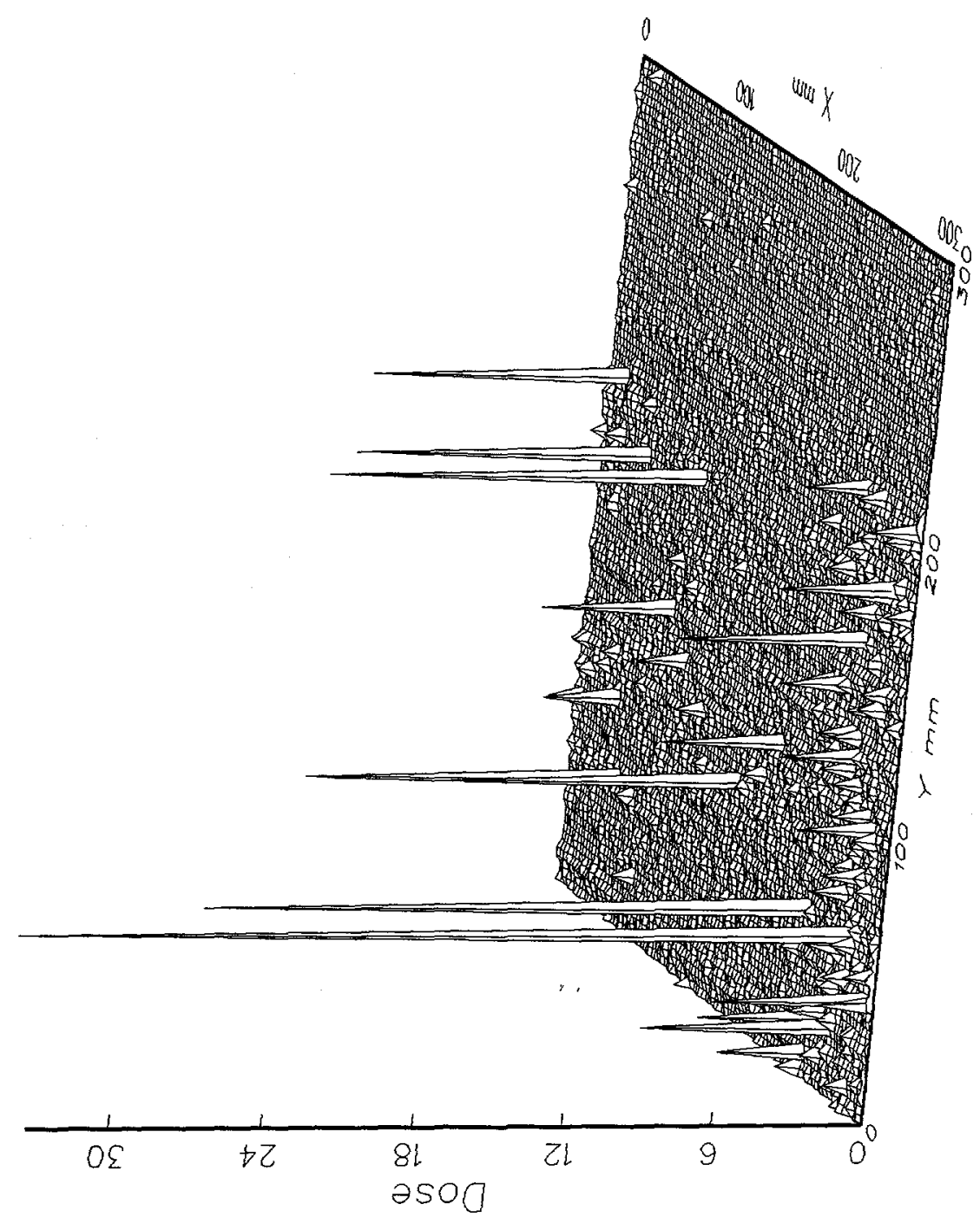

d

$\varepsilon$

응

$\wedge$

흥

은

o

声

。ำ

ठิ

온

등

흥

荬

$3 x$

สั.

文

言 实

옹

焉

음

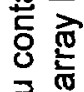

몬

응.

윽

응ำ

x

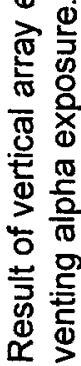

$\ddot{F}$

문 
This task, which comprises the bulk of the Phase I effort, demonstrated that this system is capable of achieving the requisite sensitivity to play a role in material and site release contamination monitoring.

\subsubsection{Particle Location and Array Resolution}

The third goal of the Phase I effort was to determine the requisite array readout resolution and array fielding conditions necessary to adequately survey a surface. The variables considered are array "stand-off" from a surface, which increases the amount of surface area viewed by an array TLD element, and the number of TLD array elements that must be evaluated in order to reliably detect the presence of a small hot particle. Of interest here are exposure time and array readout resolution. Since the array is in fact a discrete grid, with sensitive TL phosphor covering only $20 \%$ of the array area, a study based on geometrical arguments was performed to determine the detectability of a point particle emitting weakly penetrating (e.g., alpha) radiation if it is located at an interstitial area of the array. The results indicate that implementing an array standoff will adequately provide for detectability, with an increase in required exposure time. However, typically contamination is not uniformly dispersed but is generally present in patches covering at least one centimeter diameter area, ${ }^{f}$ or as particles (Sommers, 1975). This has great implications for array evaluation speed, in that the array can be read out in a manner where every second, third or fourth element can be evaluated, then coverage is accelerated accordingly (a factor of four, nine or sixteen, respectively). Then, if contamination is detected by any one element, the reader can return to this area and perform a more thorough reading, with vast time savings over straight-forward evaluation of every element. A readout algorithm exploiting this concept was developed during the Phase II effort.

These concepts were tested with the alpha emitting ${ }^{210} \mathrm{Po}$ needle, described above, which represents a point source of alpha radiation. Two types of exposure were tested. First, the tip of the $0.5 \mathrm{~mm}$ diameter needle was placed in near contact (within $1 \mathrm{~mm}$ above) with a single array element for $15 \mathrm{~min}$ utes. The result of this reading is shown in Fig. 12. The central TLD registered a signal level of about 7.8 in the figure, while the four nearest neighbors have signal averaging about one-third of this value, and the next-nearest neighbor TLDs have a signal of about one-seventh of the peak value. Secondly, the tip of the needle was placed $1 \mathrm{~mm}$ above an interstitial location (centered between four elements), and a 15 minute exposure yielded signal again about one-third of that on a centrally exposed element. This experiment was repeated with the needle tip $7.5 \mathrm{~mm}$ above the array, to check the effect of stand-off on the spread of the detectable signal. One result, with the stand off used above an interstitial position, is shown in Fig. 13. In this case, the exposure duration was 210 minutes. Generally, the stand-off results scale approximately as one would expect for inverse square dependence of the dose on distance from a point source, but the agreement is inexact due to the crude geometrical approximation for the solid angle intercepted by a circular dot that is off axis. The point to be made here is that it is not necessary to evaluate every array element, or to use a sensitivity decreasing "stand-off," in order to detect even a small particle emitting short range radiation. However, it appears prudent to read out an array at no greater than $6 \mathrm{~mm}$ resolution (cutting the reading time by a factor of four, to about 15 minutes per square foot), where at least one of the nearest elements to an interstitial particle would be evaluated for a first, quick survey. Zones on the array where localized radiation is detected can be returned to for a more thorough analysis. (In Phase II, the automated technique performs a neighborhood scan upon detection of significant signal).

fJames Durham, Battelle Pacific Northwest Laboratories, personal communication (1993). 
polar $=75$

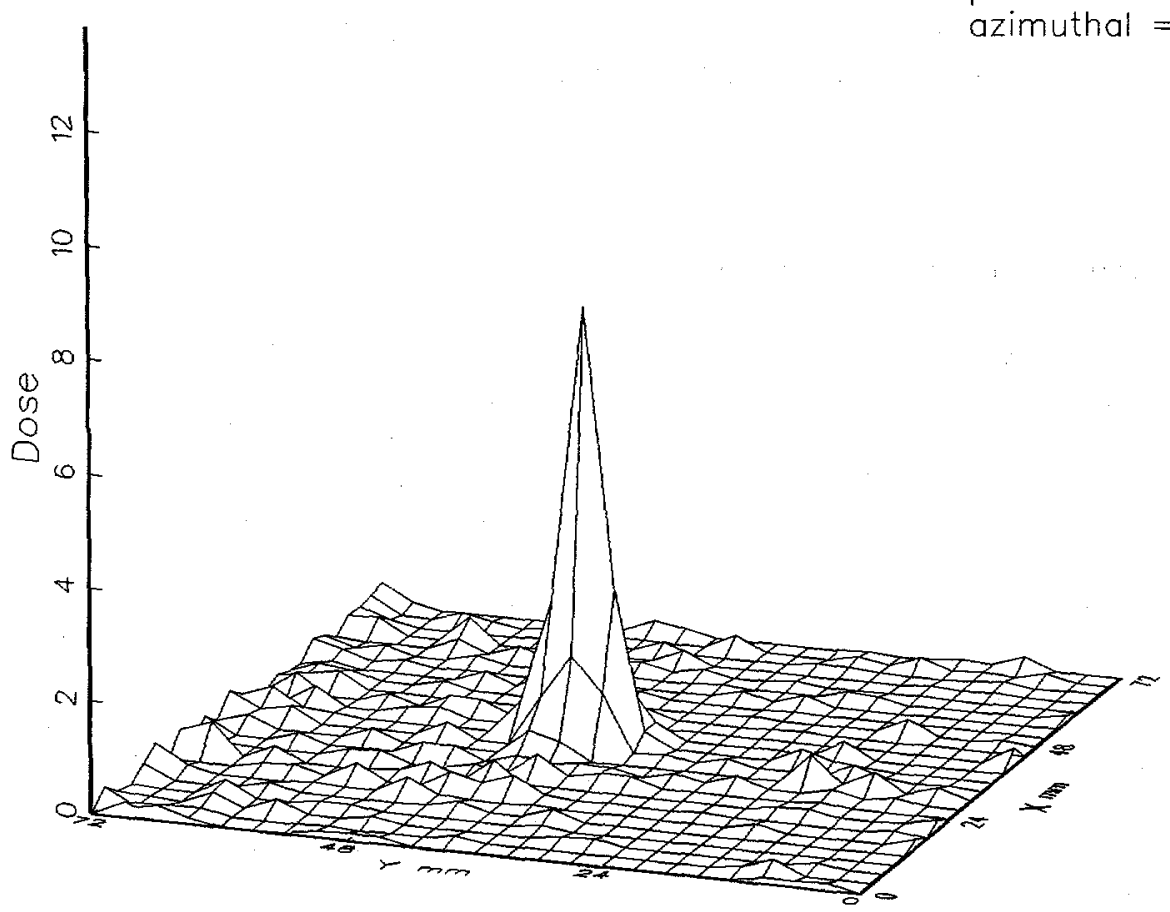

Fig. 12: $\quad$ Array response to $1010 \mathrm{dpm}, 0.5 \mathrm{~mm}$ diameter ${ }^{210} \mathrm{Po}$ needle alpha source. Source was placed $1 \mathrm{~mm}$ above and centered on a TLD element. Exposure time: $15 \mathrm{~min}$. 
Sheet number 164

Reading number 0

polar $=75$

azimuthal $=200$

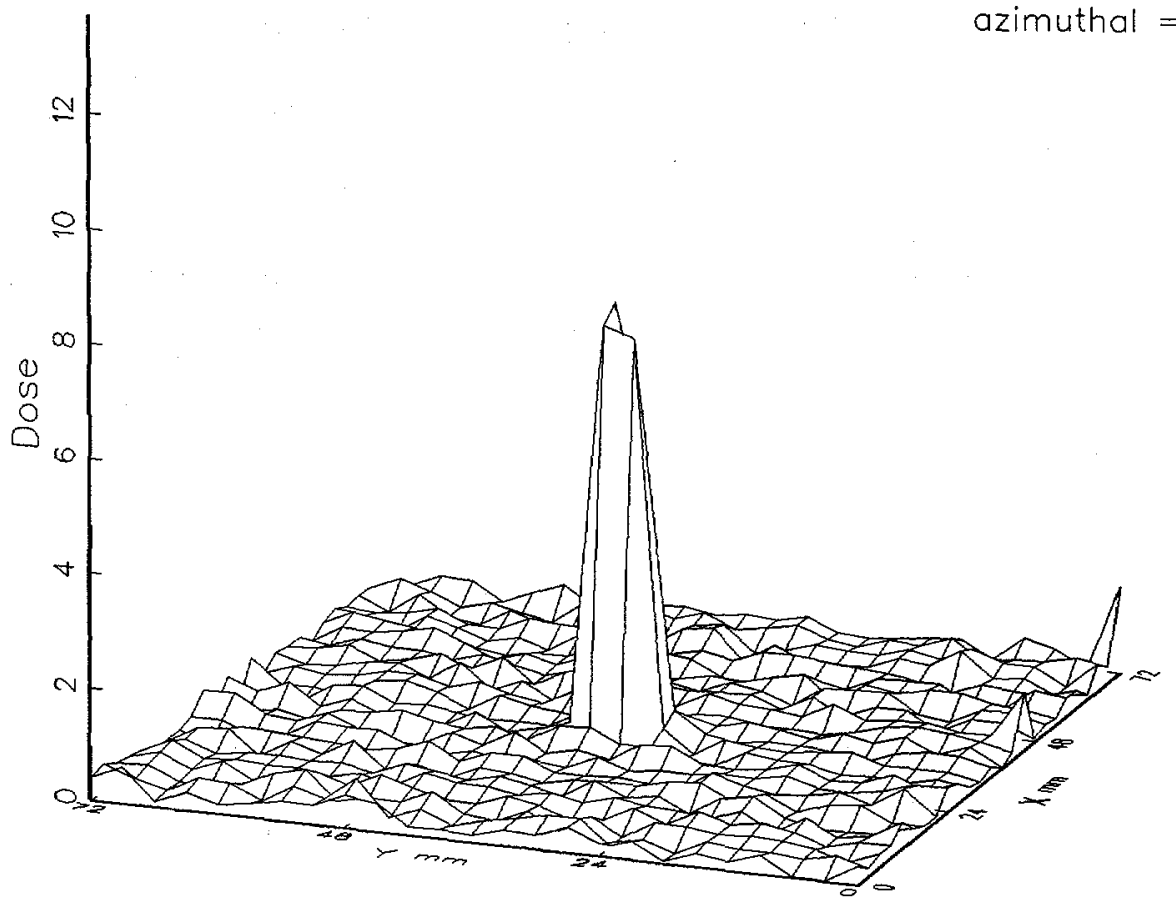

Fig. 13: $\quad$ Array response to the ${ }^{210} \mathrm{Po}$ alpha source, placed $7.5 \mathrm{~mm}$ above an interstitial array position. Exposure time: $210 \mathrm{~min}$. 


\subsubsection{Performance in Homogeneous Background Radiation}

The fourth task of the Phase I effort was to determine the detectability of localized contamination in the presence of a uniform background radiation environment. Figure 14 shows the measured result when an array was given a 90 minute exposure to the depleted ${ }^{238} \cup$ source after receiving an approximately uniform dose of $70 \mathrm{mrad}$ from a ${ }^{60} \mathrm{Co}$ source. In this case, the gamma radiation dose was equilibrated with $6.5 \mathrm{~mm}$ of acrylic (thus the signal is greater than what an uncovered array would yield given the same exposure). Even though the (signal + background)-to-background ratio is considerably less than $2: 1$, the alpha radiation is clearly apparent. It seems unlikely that an object or surface being surveyed for low-level contamination would be in an environment where this background dose would accumulate during the array exposure period, but even with this large background, the system's ability to detect localized contamination remains intact.

\subsubsection{Packaging Requirements}

The fifth task involved packaging for prevention of array contamination. The need for packaging arises from the generation of spurious indications of radioactive contamination due to dirt, dust or other foreign matter being present on an array TLD, and the generation of light induced TL. Such packaging may not be necessary if a glow curve discrimination technique is implemented. Bare arrays that were in intimate contact with contaminated soil could be successfully evaluated if they were rinsed with clean water after exposure, and a readout interrupt was performed whenever a signal above a preset threshold was measured. The "glow curve" was then displayed and a decision was made whether to accept or reject the datum based on the character of the glow curve. If the glow curve appeared to be a normal, radiation induced one, it was accepted. If not, because it lacked the characteristic glow curve shape, it was rejected. Glow curve discrimination was automated during the Phase II effort. However, light exposure can be problematic and the packaging test was performed. $A 0.9 \mathrm{mg} / \mathrm{cm}^{2}$ aluminized mylar film (which is nearly optically opaque) was tested as a packaging material. This material caused about a factor of two reduction for equivalent exposure to ${ }^{230} \mathrm{Th}$ alpha source. Thinner materials are available, reducing the attenuation effect. Clearly, packaging does not present a problem for fielding this system, requiring at most an increase in exposure time, and aluminized mylar is an inexpensive material for this purpose. Further work in evaluation of packaging materials was performed in Phase II (Sec. 3.3).

\subsubsection{Economic Feasibility}

The sixth task was to study the economic feasibility of eventually fielding the system, with respect to current contamination survey technology. As discussed above, there are applications where this system represents a unique capability for material release surveys, and can potentially exceed the state-ofthe-art for general use. Use of this system may accelerate material release rates in terms of technician labor. With regard to the possibility of performing release surveys on currently inaccessible surfaces and the implied reduction in disposal costs, the potential economic value of this system is self-evident. A comprehensive analysis of the economic viability of a product offering is currently being performed by the contractor (see Sec. 3.5.3).

\subsubsection{Hardware and Software Modifications}

The seventh and final task was to assess potential system modifications that would allow for a more rapid array evaluation rate than the current rate of about $1 \mathrm{hr} / \mathrm{ft}^{2}$ for full array read out. The options here are hardware or software engineering. While it is conceivable that the reader system can be re- 


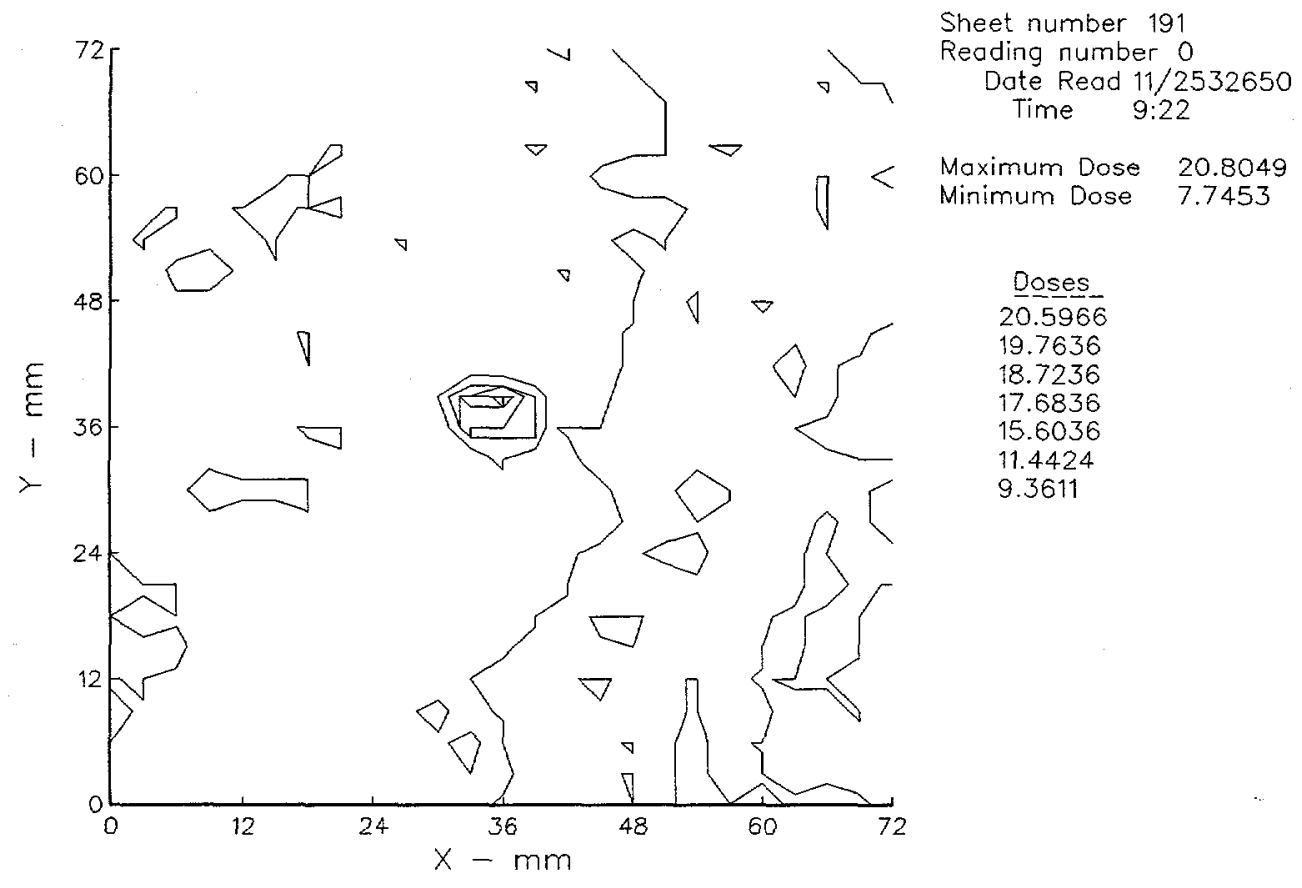

Sheet number 191

Reading number 0

polar $=75$

azimuthal $=100$

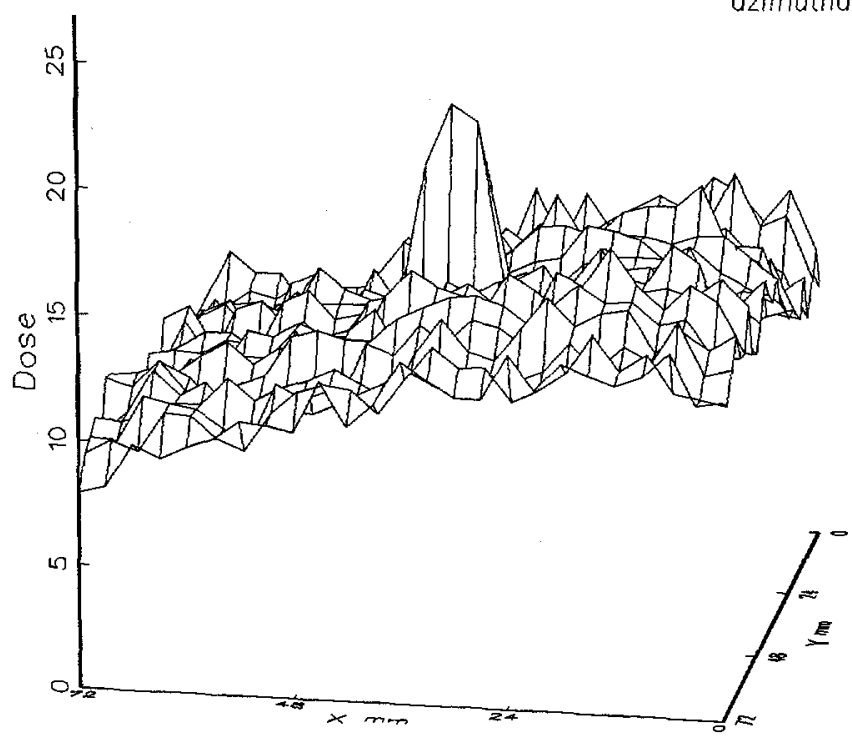

Fig. 14: Resulting output of 90 -minute exposure to the $466 \mathrm{dpm}{ }^{238} \mathrm{U}$ source, following an approximately uniform, $70 \mathrm{mrad}{ }^{60} \mathrm{Co}$ gamma dose delivered to array. 
engineered to read several array elements in parallel, there would be a large increase in system cost, leading to fabrication cost increases in the neighborhood of $\$ 50,000$, while yielding at best a factor of five in array evaluation time. It was determined that the limited Phase II funds should be invested in optimizing the sensitivity and increasing the intelligence of the present system. By applying readout algorithms that do a rapid scan of the array and then returning to areas where radioactivity was detected for a more intensive measurement, the average readout time can be reduced by approximately a factor of four. Attempting to increase speed by addition of hardware components to the reader does not appear to be warranted. In addition, the eventual price of an augmented system may exceed the combined price of two to three conventional systems with improved software. During Phase II, an algorithm was developed that allows for rapid reading of arrays. The technique requires acceptance by those performing survey work, however.

\subsection{Discussion and Conclusions for Phase I}

The measured sensitivity of the system, along with the apparently attainable low background levels, about equal to a one week exposure to alpha decay activity of $15 \mathrm{dpm} / 100 \mathrm{~cm}^{2}$, indicate that application of this system to contamination surveys for alpha and even low-energy beta emitters is feasible. Further development (as described in the remainder of this report) is required to optimize the system for this purpose. The results presented above lead to certain possibilities in the field of decontamination, remediation, decommissioning and material release surveys.

No material or facility from a nuclear installation can be unconditionally released unless its possible contamination is shown to be below certain limits (US Atomic Energy Commission). Any object exhibiting activity above release limits is classified as radioactively contaminated. Consequently, the facility cannot be reused without special radiation protection procedures or further decontamination, and the material must be disposed of as nuclear waste. In addition, an object or surface that can possibly be contaminated, but cannot be shown to be clean because conditions make a survey impossible, must similarly be assumed to be contaminated and treated accordingly. The laser-heated TLD array system, because of the flexibility of the arrays, allows for survey of surfaces previously regarded as inaccessible.

Generally speaking, survey instruments currently used in scan mode have windows for entry of radiation of several tens of square centimeters; for instance a $5 \mathrm{~cm}$ diameter "pancake" Geiger-Mueller monitor has a window of $20 \mathrm{~cm}^{2}$. In some sense, the release guideline levels are set by this fact, even though contamination is often or even typically distributed as a few small particles per thousand square feet, with activities from 2,000 to 20,000 dpm (Sommers, 1975). Yet, the reliability of these hand held monitors, used in a sweep mode, sets the practical limit for minimum detectable activity per particle at $5,000 \mathrm{dpm}$ for beta emitters (Sommers, 1975). Although this is a rather old recommendation (1975), it is clear that detection of small, hot particles may be done more reliably with this TLD array system, since a particle of 5,000 dpm in near contact with the array would register at the background signal levels discussed above in about two minutes or less, while particles having lower activity would be detected with high reliability with exposures significantly less than an hour in duration. Similar reasoning applies to alpha emitting contamination. Thus, desired release limits may not have to be compromised as a result of being impractical for standard instrumentation. In addition to being more sensitive (because of possible long term integration in a static, surface contact mode), the tedium and error of standard surveys is eliminated. A new survey methodology may ensue, with higher confidence in the results.

The most important aspect of the speed of the array system does not lie in exposure time, because exposure is a passive activity, but in evaluation rate. Passive exposures of hours or days may be preferable to active scanning if detection of lower activity levels and higher reliability of the measurement are the result. Read out strategies aimed at increasing evaluation rate were further developed. Array 
reading times of approximately 15 minutes per square foot $\left(900 \mathrm{~cm}^{2}\right)$ were obtained in Phase II when $0.6 \mathrm{~cm}$ resolution was used (see Sec. 3.2 ).

The potential of the Laser-TLD array for spatially resolved monitoring of low-level activities of nonpenetrating radiation has already been recognized by a number of persons confronted with such a measurement problem. We list here examples of several potential applications:

Department of Energy:

(1) Nevada Test Site:

Measuring and localizing residual surface contamination and determining depth distribution of ${ }^{239} \mathrm{Pu}$ in desert surface layers. Sheets can be applied to trench walls or inserted into slits in the soil surface.

(2) Oak Ridge National Laboratory, Battelle Pacific Northwest Laboratories and Hanford site:

Standard applications in monitoring surface contamination.

(3) Measurement of waste container surfaces in preparation for waste shipment.

Environmental Protection Agency:

(1) Nuclear Accident Preparedness and Response:

Measurement of surface contamination after airplane crashes involving damaged nuclear weapons

(2) Emergency Radiation Protection:

After accidents involving satellites on board rockets carrying Plutonium, standard monitoring of surfaces around launch site.

(3) Soil remediation after accidents in general.

Government, military and industrial entities will find the TLD array system useful in decommissioning fuel production and handling facilities and submarines, for instance, and in verifying compliance in decommissioning privately held and publicly owned power generating reactors. Commercial reactor owners and decommissioning contractors will find the system useful in helping comply with residual contamination standards. Any facility, whether public or privately held, such as universities or other research institutions, or producers of radiation sources for medicine, research or industry, can potentially benefit from these developments when faced with a decontamination or decommissioning problem. In addition, the research may be fruitful in providing environmental monitoring capability for a health physics program in operating fuel production and processing facilities.

In summary, the Phase I effort has revealed unique capabilities of this system for material and facility release and site restoration. This system has potential to exceed the standard methodology in sensitivity to residual activity. In Phase II, the capabilities described above were improved in general, with a vast reduction in required exposure time for detection of contamination at release guideline limits. 


\section{PHASE II DEVELOPMENTS AND IMPROVEMENTS}

\subsection{Introduction}

Using the Phase I results as background, a Phase II project was proposed and funded. The goals and objectives that were originally proposed involved optimization of the system's ability to detect radiation through hardware, software and TLD array improvements, increasing system reading speed and enhancing "intelligence," developing a suitable protocol for system deployment, and demonstrating the system performance with all improvements incorporated during a set of field tests in collaboration with contamination measurement experts. The eight tasks (as proposed) specifically listed were:

1) Optimize the raw sensitivity of the instrument to radioactive contamination by re-engineering the thermoluminescence detecting optical system and electronic signal handling system components. This work will be performed on the Phase I test-bed reader.

2) Optimize the detectivity of contamination through intelligent data analysis and reduction of spurious, false indications through software engineering similar to image processing, used to enhance data in a wide variety of applications. Use automation to speed up reading, while providing for high resolution coverage of contaminated areas discovered with arrays. Develop algorithms to provide calculation of specific activity levels using the measurement results.

3) Improve and finalize array fabrication technique, based on stencil printing, for maximum sensitivity, uniformity and minimum non-radiation induced background signal. Create array packaging technique that is inexpensive and effective, with minimal radiation attenuation and maximum protection of array from light exposure and soiling.

4) Intensively test the improvements of Tasks 1-3, and demonstrate unique capabilities of the system. Calibrated sources and "calibrated" contamination zones (field testing) will be used to test and verify performance at acceptably low levels of contamination.

5) Establish a suitable protocol for contamination surveys conducted with this system, based on the above test results, in collaboration with appropriate experts.

6) After analysis of test results and expert input, modify the system design and fabricate a new reader according to new design specifications, with software enhancements as appropriate.

7) Test new system to verify performance, and correct flaws and deficiencies. Repeat field tests, to demonstrate field readiness of system.

8) Analyze final results and prepare final report.

Not all of these objectives could be met during the course of the project. The most significant impediment to project progress was the appearance of a very large spurious light emission from the arrays fabricated with silicone binder. Details of the problem are discussed below. This emission eventually became overwhelmingly large, with the implication that if it could not be solved, there would be no point in continuing with any other tasks. Therefore, virtually all available resources were dedicated to the solution 
of this problem until a satisfactory resolution could be achieved. Since there was no indication before the beginning of this phase that a problem of this magnitude existed, no allowance for solving it was scheduled or budgeted, and the impact on the progress of the other tasks was very large. In turn, opportunities for field testing were missed, and other cooperative efforts were delayed or postponed until the spurious emission could be shown to be sufficiently under control such that it would not be the cause of failed experimentation. Meanwhile, a significant change in the contamination measurement research community, caused by program and budget cuts at many of the Department of Energy funded laboratories, eliminated the possibility of carrying out many of these planned activities due to the reassignment, layoff, or retirement of our collaborators. Further, acquisition of the original contractor by Keithley Instruments resulted in the loss of important project personnel due to impending relocation. For this reason, certain desirable system features could not be implemented before the conclusion of the project. Rather than dwelling on these problems further, we shall report on the level of progress attained and the status of the development of this system at the conclusion of the project period.

In the following section, the work performed related to the above tasks will be discussed, along with an assessment of its overall impact on the performance of the system.

\subsection{Phase II Technical Improvements and Developments}

The Phase II technical effort can be characterized as primarily focusing on two aspects of the problem of measuring low-level radioactive contamination. These are maximizing the measurable signal induced in the thermoluminescent phosphor by decaying nuclides, and suppressing effects that interfere with its measurement. Secondary efforts were aimed at increasing the system rate for TLD array evaluation. Thirdly, algorithms were developed for calculation of measured activity levels, and a protocol was devised, based on minimum array exposure times, for achieving the limits of detection required to meet the contamination limits specified in Table I. Intensive development testing was a component of all of these efforts, and some limited field testing was performed before all developments were fully incorporated into the system.

The TLD reader described in Section 2 was used as the foundation for implementing all physical improvements devised here. It remained fundamentally unchanged in its overall description and operation. The configuration of the phosphor arrays are significantly different in Phase II: Instead of TLD elements being deposited as $1.5 \mathrm{~mm}$ diameter spots, the "arrays" now consist of continuous layers of phosphor and binder, yielding discrete array elements having $4 \mathrm{~mm}^{2}$ of area rather than $1.77 \mathrm{~mm}^{2}$. Other specific enhancements are discussed. During the course of the project, these enhancements did not require fabrication of an entirely new reader, nor did funding allow it in view of the resources expended on solution of the material problem with the TLD arrays.

The essential concern during this project was technology development, and only a small amount of effort was expended on the economics of its implementation. However, these concerns cannot be overlooked. Discussions with experts concerned with measurement of surface contamination generally resulted in a measurement cost per unit surface area. Ultimately, the value of an object as a releasable item needs to be in excess of the cost required to ascertain its level of contamination. The disposal cost needs to be factored into this equation as well. 


\subsubsection{Sensitivity Enhancements}

Three opto-electronicenhancements to the test reader were developed for the purpose of improving detection of the thermoluminescence signal. The first invoived replacement of the photomultiplier tube and its high voltage power supply with a packaged device available from Hamamatsu, Inc. The second involved the addition of photon counting capability to the reader electronics and software. Third, the fiber optic signal pickup module was improved for sensitivity and ability to read full-size arrays.

The packaged light detection module is a Hamamatsu model HC120-01. It contains a photomultiplier tube (PMT), a high voltage supply, and a signal preamplifier. Using this package resulted in improved noise characteristics of the light signal. A circuit modification was made in the reader electronics to narrow the single photon pulses such that rates to 125,000 pulses per second are counted and remain linear with light intensity. An adjustable lower level discriminator is part of the photon counting circuit. In the reader, the signal acquisition system has two parallel channels: one involving measurement of the PMT DC voltage signal, for high signal levels, and the other a pulse counting circuit for low level signal. Both channels are monitored simultaneously, and the information is used in measurement of the TL signal.

In Phase I, we devised and tested an improvement to the light collecting optical fiber bundle that collects the thermoluminescence emitted by the phosphor and transmits it to the PMT photocathode. This bundle consisted of eight 600 micron diameter optical fibers surrounding the emitting array element. Although this provided an increase in light collection by about a factor of 12 over the standard configuration (the original configuration at the beginning of Phase I), the stiffness of the fibers in the bundle and the routing of the bundle in the reader restricted the area of an array that could be evaluated. The obvious solution is to use smaller diameter fibers, but this will reduce the amount of light collected per fiber by the ratio equal to the square of their relative diameters (the amount of light entering a fiber is proportional to its cross sectional area). Further, the number of fibers that can be included in such a design is not arbitrary due to fabrication considerations. We found that twelve 400 micron core diameter fibers can be used, with an overall reduction of collection by about $45 \%$ in comparison with the eight larger fibers. This represents a theoretical increase of about a factor of 7 over the standard bundle, however, and the 400 micron fibers are approximately three times more flexible than the 600 micron diameter fibers. This approach was implemented. Measured light collection improvementover the standard configuration exceeded a factor of six. Further, full size, $30 \mathrm{~cm}$ square TLD arrays are readable with this new bundle.

Along with photon counting, other signal processing techniques were developed and applied to the thermoluminescencesignal. The principal objective was to further compensate for the noise induced in the signal channels by the $\mathrm{CO}_{2}$ laser power supply. Spikes appear in the unprocessed PMT signal due to switching of high power to the laser, and some digital noise also leaks into the signal channel. We applied an auto-correlationalgorithm to the PMT DC voltage signal channel in order to reduce the influence of these spikes on low level signals. This technique does not significantly alter the low frequency characteristics of the TL glow curve. Certain other required corrections were also developed involving signal offset correction. One hardware modification associated with the reader's array transport mechanism was implemented for the purpose of increasing reader processing speed. The efficacy of these improvements will be discussed below in conjunction with other improvements yet to be described.

\subsubsection{Specific Software Developments}

The object of the second task listed above was development of software for the overall purpose of improving reader performance in detecting radioactive contamination. The areas covered include reader speed increase through course/fine array scanning, automated rejection of spurious glow curve signals, array 
data analysis for enhancing detection of localized radiation, algorithms for computation of detected activity levels, and background subtraction techniques for the purpose of extracting the radiation signal from the instrument background (in Phase I, two readings of an array were required to derive the net radiation signal). The last of these was greatly aided by all other improvements leading to reduction in instrument background.

The software component of the system consists of two parts. The actual reader control and data acquisition software is run internally on a microprocessorusing the FORTH computer language. While advantageous for hardware control applications (even more so at the inception of the Phase I project and earlier, when the system was initially developed), it is nearly unusable for development of suitable data analysis and visualization routines. These tasks are handled on a PC using MATLAB, a scientific analysis software package (The Mathworks, Inc., Natick, MA), purchased under Phase II sponsorship. A minor part of the software runs under a third graphics program, GraphiC, (Scientific Endeavors Corp., Kingston, TN). This latter program is a holdover from earlier developments, now used only to display glow curves during the array reading process. MATLAB contains numerous preprogrammed routines for handling large data arrays, a feature we exploited to great advantage with the large, 10,201 TLD element arrays.

Due to the turn of events during this project, not all of the envisioned software developments could be fully implemented. We focused on those deemed most important for basic system performance that could be implemented before the departure of key personnel. Those that were viewed as requiring less specific laser TLD or FORTH expertise by the programmer were deferred, to be implemented as time permitted or until further development occurred in follow-on work. All of the deferred programming can be accomplished in MATLAB, an easier task than programming in the FORTH language.

\subsubsection{Automated Glow Curve Analysis}

The TLD arrays may become contaminated by particulate matter during fabrication or as a result of use. This particulate may emit light, most usually incandescence, if heated by the laser beam. Occasionally during reading, signals contain features that are not characteristic of TL glow curves, and these may have an adverse effect on the measurement of very low-level contamination. During Phase l, a read interrupt routine was invoked whenever a selectable level of glow curve signal was exceeded, and the operator could accept or reject the datum based on expert opinion. If rejected, the signal value was replaced in the array with the average of its four nearest neighbors. This mode requires constant operator attention to minimize expansion of array evaluation time. The goal of this subtask was to automate this process. A technique was developed by which the reader displays and analyzes any glow curve having a signal level above an operator set threshold, which can be arbitrarily low. Using three criteria based on characteristic glow curve behavior, the reader automatically accepts or rejects the datum for that array element. In the current state of development, all analyzed glow curves are stored for review during or at the end of an array reading, and the operator can override the reader's decision of acceptance/rejection. This review is a fairly quick process. The threshold for glow curve analysis may be changed during the course of an array readout. The decision criteria are based on time dependent characteristics of the glow curve, which are themselves characteristic of the TL phosphor in use and the heating program. Changes in the heating program or phosphor or array characteristics necessitate adjustment of the decision criteria.

After fine tuning the technique, we have found that the decision criteria are very accurate in matching an expert judgment on the acceptability of a glow curve. The criteria were developed on the assumption that low-level radiation induced glow curves are to be analyzed. Part of the developmentwas in the observation of thousands of glow curves that were certainly due to small radiation doses, even though they may lack the look of textbook example TL glow curves. Figure 15 shows an example of a computer screen displaying glow curves analyzed during an array reading. In this example, several of the glow curves were rejected by the algorithm, as denoted by one or more F's (failures) in the panels. Compare the failed curves with those in the third row, which are archetypal for the heating program used. 
주ㅇㅝㅗ

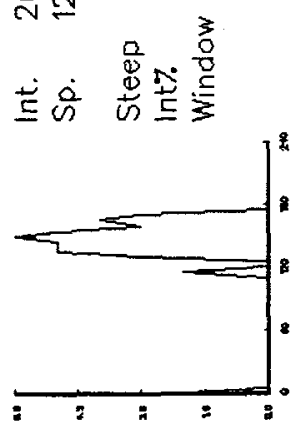

\pm
7
0
0
0

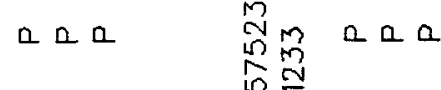

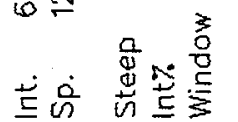

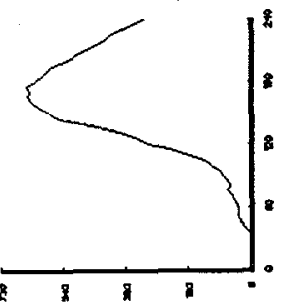

in

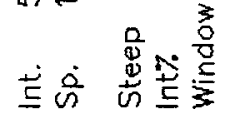

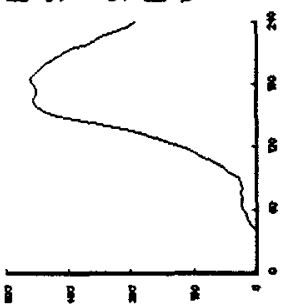

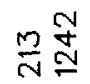

它离离点愛

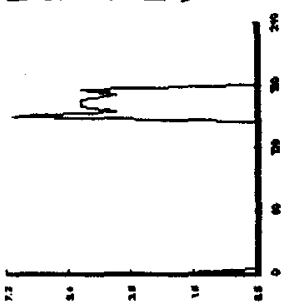

অ্ّ

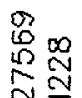

$a 0 a$

$\stackrel{+}{\infty}$ a a a

Е

落离点点

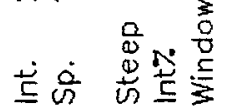

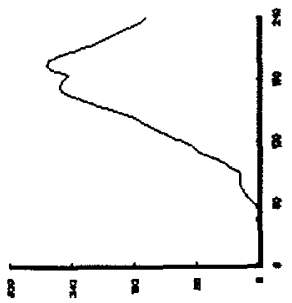

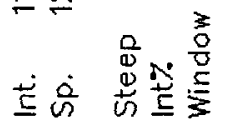

蛋昂

a a

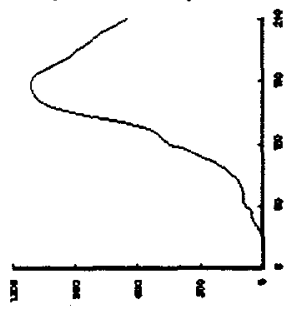

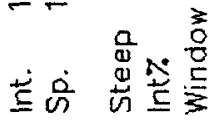
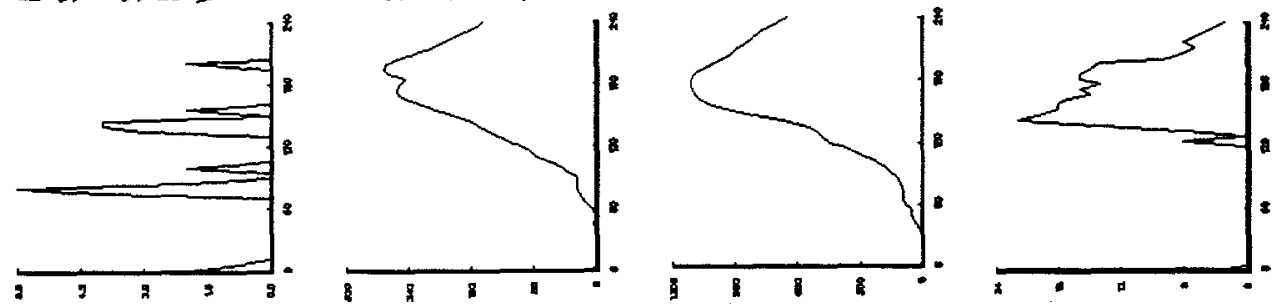

Nㅗ용

ㄷㄴ

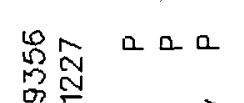

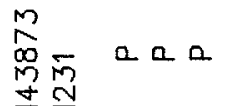

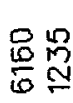

a 0

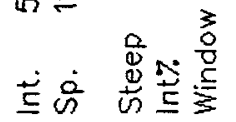

on

迹总咅

$\forall \mathbb{V}$

离

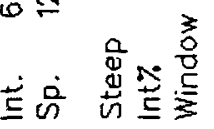
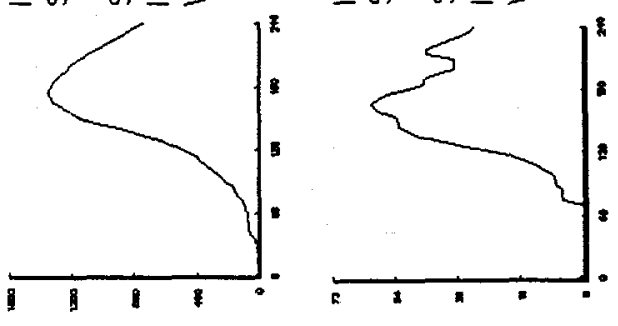

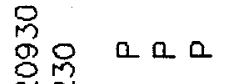

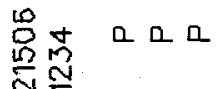

品 零怘

므뭉

$\stackrel{0}{N} \stackrel{0}{ \pm}$

a. 0.

옹

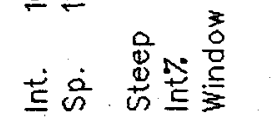

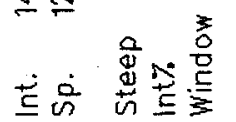
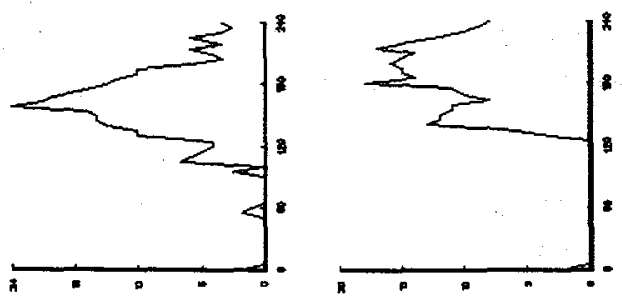

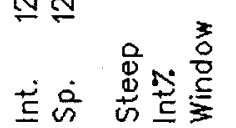

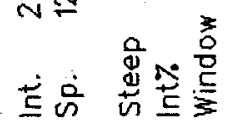
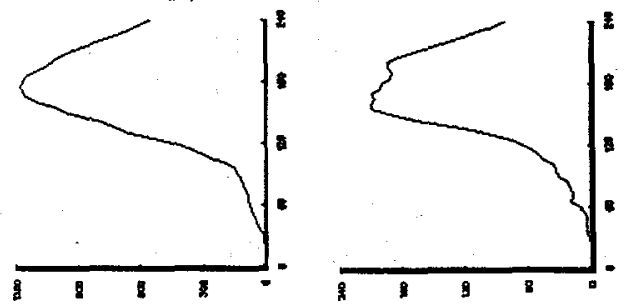

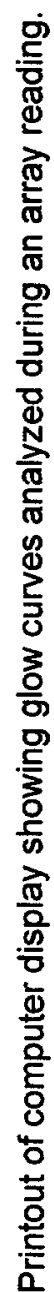


The utility of this function is large. It primarily serves as a reassurance that any readings of significant level are indicative of the presence of radioactive material, and not of a spurious nature. One problem encountered during the course of the project cannot be dealt with using this technique, however. That is the effect of the discharge of static electricity into the TLD layer, since this phenomenon does induce a glow curve characteristic of nuclear radiation. This is to be expected, since high energy ions or electrons are stopping in the phosphor, just as the case with incident alpha or beta particles. See 3.2.3.3 for additional electrostatic discharge issues related to array packaging.

\subsubsection{Array Coarse/Fine Scanning}

In order to speed up the surface measurement process using this system, an algorithm was developed to survey an array at $6 \mathrm{~mm}$ reading resolution, and then to search around the neighborhood of any measured array elements that indicate a significant signal level. The array elements consist of a read area of $2 \mathrm{~mm} \times 2 \mathrm{~mm}$ of phosphor, centered on a $3 \mathrm{~mm} \times 3 \mathrm{~mm}$ grid. The concept is to provide a sample of the surface measurement, and then to follow up on any suspicious looking samples. Sampling at greater intervals, e.g., $12 \mathrm{~mm}$ grid spacing, does not seem prudent in the case of surveying for short range radiation. The potential benefit to be gained is the rapid evaluation of arrays exposed to "clean" surfaces, as perhaps may be applicable in decontamination verification surveys.

Along with other minor hardware modifications associated with this task, the processing time required to sample a $30 \mathrm{~cm} \times 30 \mathrm{~cm}$ area of clean surface is 18 minutes, in contrast with 60 minutes required to read every array element on the $3 \mathrm{~mm}$ square grid. In the case where significantcontamination is found distributed over the array during the coarse survey, any time saving is reduced as the neighborhood search function will be invoked so frequently that it may be more efficient to read the array at full resolution. The dividing line on this time efficiency vs. contamination level is undetermined.

Three approaches were investigated, which we have termed Reiterative 3, Reiterative 5 and Nonreiterative 5 neighborhood scanning. The neighborhood scans are triggered whenever one of the pixels in the coarse grid has a signal in excess of an operator set threshold. The Reiterative 3 routine will search the $3 \times 3$ subset around each dosimeter read which exceeds the threshold. This $3 \times 3$ subset will be read at $3 \mathrm{~mm}$ resolution. For each of the dosimeters read within the $3 \times 3$ subset which also exceeds the threshold, an additional $3 \times 3$ subset will be read for each of these centered on the dosimeter which exceeded the threshold. Reiterative 5 works in a similar fashion except that the subset read for each dosimeter which exceeds the user set threshold is now $5 \times 5$. The Non-reiterative 5 routine differs from the previous two routines. The Non-reiterative 5 routine will only perform the initial search of the $5 \times 5$ subset centered on the dosimeter which exceeds the threshold. Each of the dosimeters within the $5 \times 5$ subset will not be compared to the threshold. Their readings are simply stored, and the coarse scan is resumed, until a new dosimeter exceeds threshold. Note that no TLD element is ever read twice; if an element has already been or is to be read during the coarse scan, this fact is accounted for in the decision whether to read an element during a neighborhood scan.

The Reiterative 5 routine appeared to show problems if a sheet contains a significant amount of radiation exposure. The routine, in this case, will read in a wandering or chaotic manner without improved coverage. The Reiterative 3 and the Non-reiterative 5 routines showed a significant improvement in readout time without sacrificing or missing areas of interest. During development testing, the Non-reiterative 5 routine provided slightly better neighborhood coverage and speed, and is immune to wandering. This was adopted for further testing, discussed below, where we present results of this algorithm's capability of detecting variously distributed radiation fields. 
The usefulness of this capability has not been determined as its acceptance is subject to analysis by persons concerned with development of survey methodology. Nevertheless, this capability was developed and tested, and exists for use at the option of any customer who may adopt this system into their survey measurement program.

\subsubsection{StatisticalAnalysis of Results}

Preprogrammed MATLAB algorithms for calculation and display of statistical analyses of array reading results were customized for this application. The entire array or contiguous, rectangular regions can be analyzed. This greatly enhanced the ability to analyze results and perform background subtractions from reading results. Although not fully automated (i.e., a determination of background and its subtraction is not automated), the operator may utilize the built-in functions to easily perform arithmetical operations on the entire array. This represents much progress, and eliminates the need to perform second array readings for subtraction of instrument background.

\subsubsection{Convolution of Readings For Calculating Contamination Surface Density}

Early in the project, generally well-known image processing techniques (Russ, 1995) were investjgated for the purpose of enhancing the detection of small scale contamination zones. During project progress, it became apparent that the primary concern was measuring contamination in terms of activity detected within an enclosed area. The measured values are used to determine whether a surface is acceptably clean or must be further processed or held in a controlled area. Generally, the desired results are expressed as those in Table I above, i.e., contamination in units of $d p m / 100 \mathrm{~cm}^{2}$. A technique was developed to obtain this information by using the available convolution algorithms in MATLAB. In the current application, a specific type of convolution kernel is used to transform the reader output matrix into a form that represents the total number of nuclear disintegrations within a fixed-size window. This approximates the result that would be obtained by scanning an active detector, of the same window size, arbitrarily slowly over the same area covered by the TLD sheet.

For purposes of this discussion, a convolution consists of summing the product of data array elements with the corresponding elements of a kernel over all possible kernel positions. The result is a new array which represents a transformation of the original array. In the present case, using two-dimensional data derived from sheet readings, which we can denote as the position dependent thermoluminescent signal, $S(X, y)$, stored in an array format, the convolution of this array with a kernel $K(X, y ; X, Y)$ yields a new function $S^{\prime}(X, Y)$ according to the following

$$
S^{\prime}(X, Y)=\Sigma \Sigma S(x, y) K(x, y ; X, Y)
$$

where the sums occur for all values of $x$ and $y$ within the kernel, and are performed for all positions $X, Y$. In essence, the kernel is overlaid on $S$ at all possible positions of $X$ and $Y$. The kernel typically represents a weighted average. 
Consider the example of a kernel consisting of a matrix with elements equal to the number 1 , which spans a square shaped area of $100 \mathrm{~cm}^{2}$ on the original data set. The original data is a $101 \times 101$ element array spanning a $30 \mathrm{~cm} \times 30 \mathrm{~cm}$ area. The kernel is a $33 \times 33$ matrix of 1 's covering $10 \mathrm{~cm} \times 10$ $\mathrm{cm}$,

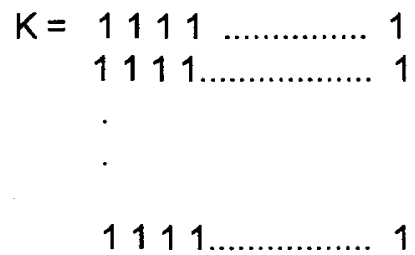

The output array value at any point in the $X, Y$ plane represents the sum total of the thermoluminescence signal for all array elements contained within the $100 \mathrm{~cm}^{2}$ square centered on that point in the original data set. The output array represents the measure of the total signal within all possible $100 \mathrm{~cm}^{2}$ square areas whose center could be contained within the boundaries of the TLD array. When properly calibrated, such analysis would reveal whether any square of area $100 \mathrm{~cm}^{2}$ contained activity above a threshold, e.g., $500 \mathrm{dpm}$. The shape of the area is fixed in any one analysis. Kernels can be made in any shape or size, however. The result represents literally hundreds of overlapping samples of the readings on an array. Note that kernels must have odd dimension so that there is an element or spot at the kernel center. The coordinate of this element gives the position of the operation output value on the original space of the TLD sheet array.

The convoluted output of an array reading can be thought of as scanning a detector, having an active area or window in the size and shape of the kernel, arbitrarily slowly over the surface area covered by the TLD array. However, in this case, the detector window is actually in static contact with a fixed area for the entire period the array is in contact with the surface. The graphical output represents an approximation of what would be obtained from a scanning survey performed with a fixed window area active instrument, as patches of contamination are approached, covered and recede from the detector window. But, the benefit of a long count time, static survey are also obtained. The convolution kernel can be specified to be any size or shape that is convenient.

It is conceivable that with fixed area active survey monitors that nonoverlapping adjacent samples of a contamination zone could pass the criteria of being under $X X \mathrm{dpm} / 100 \mathrm{~cm}^{2}$. But, if the detector were positioned differently, it would have found a zone with activity greater than $X X \mathrm{dpm}$. This scenario is avoided with the array convolution method. A suitable size and shape will need to be determined, however. Given that analyzing arbitrarily small areas is feasible with the TLD array technology, the unit of area size for a contamination specification could be readjusted accordingly, say perhaps to $50 \mathrm{dpm} / 10 \mathrm{~cm}^{2}$ instead of $500 \mathrm{dpm} / 100 \mathrm{~cm}^{2}$, for example.

A problem with handling the edges of TLD array data arises when part of the convolution kernel falls outside the array boundaries. This is really only a matter of visual interpretation. The valid region of a convolution output consists of that collection of array points for which the kernel is overlapping valid input data. An output option that returns only the valid array is available, but this is visually confusing because a $30 \mathrm{~cm} \times 30 \mathrm{~cm}$ array returns a smaller array, diminished in size by the dimensions of the kernel (half the kernel width as it scans each edge). For this reason, a same-sized array may be output wherein the missing data beyond the TLD array edges is padded with zeroes. This has the effect of placing the 
convolution output on the same size axes as the input data. The user must recognize that the entire output is not valid, however.

Examples of array processing with various convolution kernels are shown in Section 3.3.

\subsubsection{Computation of Surface Contamination}

Once an array has been read, processing with appropriate convolution kernels can yield output calibratable to specific nuclides. Once an appropriate calibration is obtained, it may be applied to the processed output to obtain surface activity levels, and this in turn may be statistically analyzed to yield maximum, mean, and various other statistics in the desired units, e.g., dpm/100 $\mathrm{cm}^{2}$. The mean value would represent the average surface contamination measure for all possible positions of the kernel window, as does the maximum. Note that for a given kernel, these results apply only to the size and shape of that kernel.

\subsubsection{TLD Array Development}

As conceived in the Phase Il work plan, development effort on fabrication of the TLD arrays was not expected to be a major undertaking. Primarily, we planned on a small amount of experimentation on the formulation of the silicone binder material used to bond phosphor grains to the substrates. Additionally, we were to develop stencil printing methodology, and Mylar array packaging. The overall goal of this task was to develop highly uniform, stenciled arrays with low background signal (by reducing the nonradiation induced thermoluminescence(NRI-TL) contributed by the silicone).

\subsubsection{Array Fabrication Binder Material}

It had been known that the silicone binder contributed at least a weak NRI-TL since this material has been used in this application for many years. The effect was weak but inconsistentin the Phase I work of this project. For all practical purposes, this effect came to dominate both the light emission from the arrays and the project as a whole. The observed signal could grow to be as large as a signal induced by a 20 rad dose of gamma radiation, clearly swamping the signals induced by low-level surface contamination. After several months of investigation, it came to be realized that the effect, which came and went, was correlated with the work-site air conditioning system cycling on and off. This was verified to an absolute degree because we could control it; we could read silicone-based TLD arrays without observing the effect by simply turning off the ventilation system manually, and cause it to appear by turning it on. The air supply was suspected of being contaminated, and was tested by analysis in a gas chromatograph. Exposing arrays to concentrated samples of the identified substances did not induce the effect. However, turning off the air conditioning system for extended periods was not a viable option for solving the problem. The problem was not traceable to a change in the silicone formulation by the manufacturer, as old arrays that hadn't exhibited the NRI-TL to an overwhelming degree now did so.

No satisfactory explanation as to the mechanism behind this observation was ever obtained. It is the opinion of the principal investigator that the only new contributing factor that could be responsible for this sudden onset of overwhelming NRI-TL is the fact that the reader enclosure was changed. When the Phase I work occurred, the reader had a hand made prototype enclosure, which was changed during Phase II to a cabinet that was designed to be made in quantity. In the earlier enclosure, the chamber in the reader containing the array was sealed, such that the array sat in dead air during the reading process. In the new design, air was allowed to flow in the reading chamber. The cabinet materials were not the agent, as the effect could be controlled by the ventilation on/off switch. As this air flow in the reader array chamber occurred whether the ventilation system was activated or not, something appeared to be transported into the room in the ventilation air. The outdoor air was tested to see whether the agent was introduced in the AVC system. 
Whatever the ultimate cause, it existed in the outdoor air, but dissipated rapidly when brought in through the AlC. While it appeared to be somewhat localized to the work-site environs (exposing arrays to outdoor air at other locations did not always cause the signal to appear), it is not specific to that location. It now seems that this phenomenon was responsible for the low level NRI-TL that was to be solved as proposed, except that arrays were only briefly exposed to room air during normal use, and during the exposure periods, the AVC may have been off or on. Hence, the observation of its inconsistent presence. The problem with the new cabinet was that the signal would grow during the readout process (requiring about 60 minutes for full sized arrays), and not sufficiently reproducibly to be accounted for with some subtraction technique. Attempts to seal the reading chamber were effective, but not entirely successful. A reduction by two orders of magnitude was obtained, but this was insufficient. The problem definitely resided in the silicone binder, and a substitute binder had to be found.

Only a few materials exist that might be suitable for this application. All that became known to us were tried, and most of them were some sort of differently formulated silicone, which would exhibit the same effect. Finally, we obtained a printable polyimide binder from Epoxy Technology, Inc. (Billerica, MA) that was usable and appears to be totally immune to the agent producing the problem in the silicone.

This being the case, much work adapting this new material to our production process needed to be done. Although the polyimide binder "solved" the problem, we nevertheless found that this material has certain characteristics that make it less than ideal. Primarily, printed phosphor layers turn out with a yellowbrown color (the phosphor is white) that we feared would attenuate the TL emission, and it tends to darken with repeated use as the arrays are oven annealed at $235^{\circ} \mathrm{C}$ between uses. This leads to slight reduction in sensitivity with each use (about $2 \%$ per reading), but this could not be stabilized, even with annealing in an oxygen free atmosphere. The arrays were spectroscopically tested for relative attenuation of the important emission bands of the $\mathrm{CaSO}_{4}$ : Tm phosphor. An approximate loss of $20 \%$ in absolute sensitivity was observed in relation to the same phosphor in silicone binder, but this loss was more than compensated by the elimination of the NRI-TL. The relative magnitude of the silicone problem can be approximated as equivalent to the signal induced in the phosphor by a 20 rad dose from gamma radiation. With the polyimide, the average background equivalent signal is on the order of a millirad of gamma dose. The comparison is shown in Figs. 16 and 17. In Fig. 16, a silicone based-array is shown as read after a 50 minute exposure to the air in the laboratory. In Fig. 17, an equivalently exposed, polyimide-based array is shown.

With this level of improvement, work on the other project tasks could proceed. This success also satisfied the need to eliminate array related background signal. Further work on eliminating or coping with the sensitivity degradation is required for commercialization of this system.

\subsubsection{Array Fabrication Configuration}

Development of array fabrication using stencils rather than screens was eventually dropped from the project, largely because of the expense involved in the fabrication of stencils for large, $30 \mathrm{~cm}$ arrays and the incompatibility of our large array printer with stencils.

Rather, the large $30 \mathrm{~cm} \times 30 \mathrm{~cm}$ "arrays" were fabricated as single, continuous phosphor/binder layers. The motivation for this is two-fold: historical difficulties in producing the large-area discrete arrays consistently meeting critical alignment criteria in the reader, and an enhancement of sensitivity through heating of greater phosphor area. The continuous sheets are still read as if they consisted of discrete TLD spots, but more than twice as much (a factor of 2.3 more) phosphor area is heated by the $2 \mathrm{~mm}$ square laser beam than in the $1.5 \mathrm{~mm}$ diameter discrete spots, increasing sensitivity in about the same proportion. This type of sheet also more thoroughly surveys the measured surface without increasing the evaluation time. Further, continuous layer sheets are simpler to fabricate in terms of production set-up effort (perhaps one full person-day can be saved per production run because printer alignment is much less 


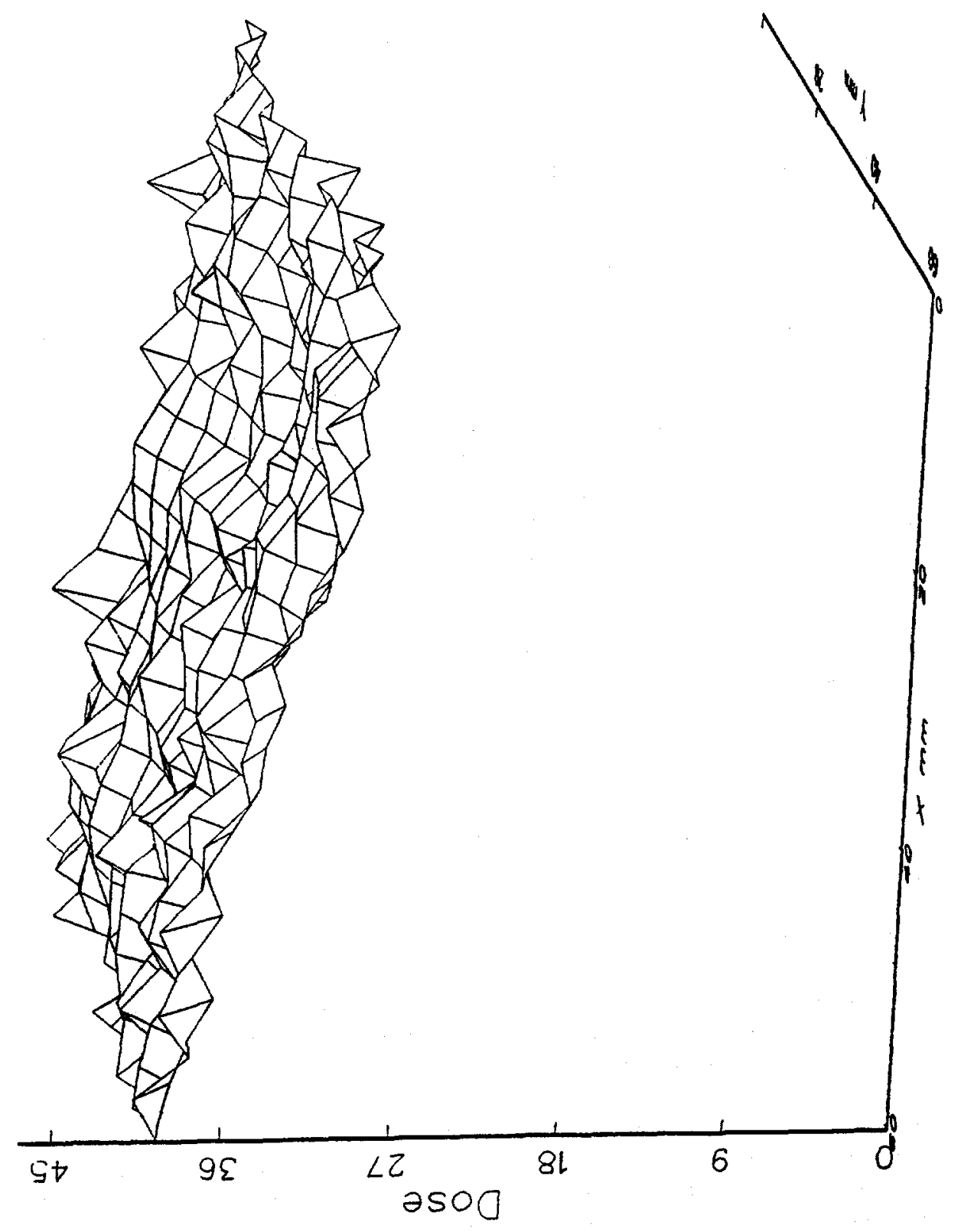

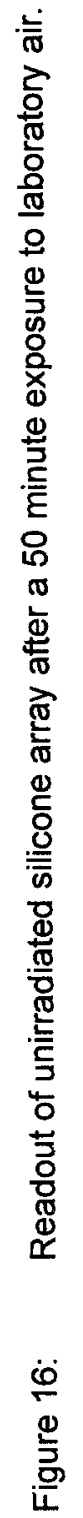




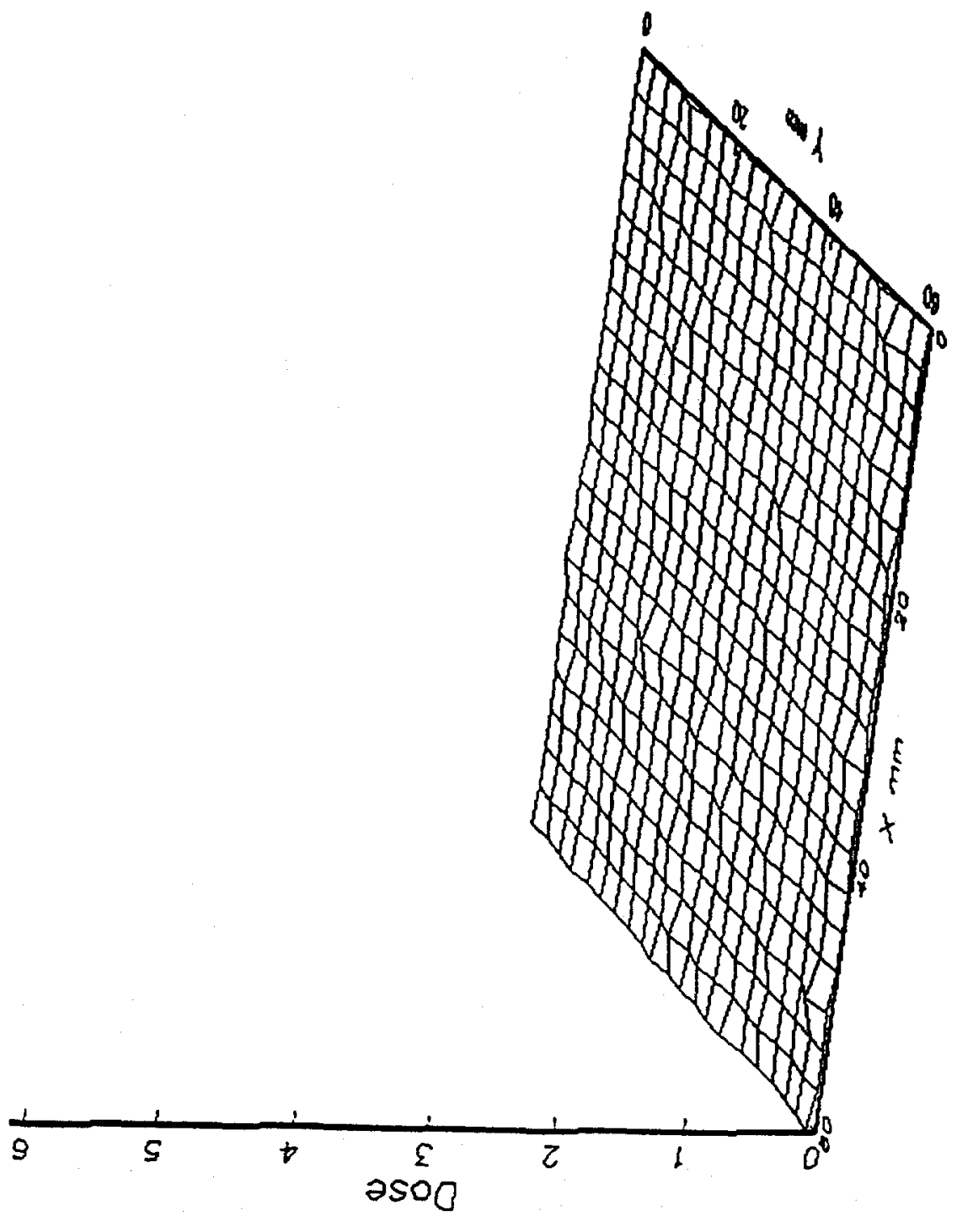

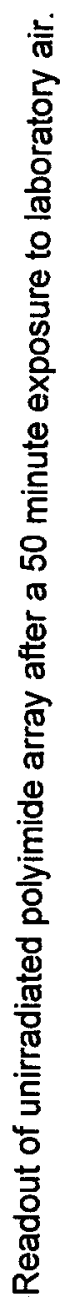

$\frac{\stackrel{N}{\sigma}}{\stackrel{ \pm}{5}}$ 
critical), reducing eventual cost of these sheets. Higher sensitivity also implies a proportional reduction in exposure time for achieving a defined survey protocol. The actual surface area of the TLD sheets or arrays evaluated amounts to $408 \mathrm{~cm}^{2}$ out of the total $912 \mathrm{~cm}^{2}$ area covered by phosphor on the substrate. The $4 \mathrm{~mm}^{2}$ heated pixels are separated by a strip of $1 \mathrm{~mm}$ width. This factor was not considered explicitly in the following work, but it is unlikely that a speck of significantly active surface contamination will be completely undetected with this configuration. Henceforth, these TLD detectors may be referred to as arrays or sheets, and the surface unit of heated phosphor may be referred to as array element or pixel.

Part of this task was to develop the fabrication process such that arrays would be produced with uniform response from pixel to pixel, in order to eliminate the need for array calibration, essentially a pixel response normalization. This could not be achieved with the existing printing equipment, and procurement of adequate equipment was beyond the funding scope of this project. Therefore, arrays used in testing have both systematic and random variation in pixel response to radiation dose. This can be dealt with by array calibration, in a fairly simple manner, but nevertheless, this was a task that was deferred while the fundamental work on sensitivity and background suppression, signal processing, etc., was being performed. The lack of array calibration has a lesser impact in determination of measurement capability for the case of low background radiation. However, its absence does broaden the statistical distributions used to make that determination. A greater impact is felt in the case of larger background radiation fields, as the random and systematic variations in array response cannot be normalized, causing difficulty in applying a background subtraction technique. This work will proceed as further development occurs during the efforts to commercialize this system. Some testing of the measurement of isolated alpha sources in a near uniform gamma radiation field was performed, and suggestions for improving the capability are made.

\subsubsection{Array Packaging}

Array packaging was listed as a work plan task primarily for the purpose of protecting the surface of the array from particulate contamination during exposure to dirty surfaces, or, in fact, to soil. The obvious choice is to cover the array surface with an appropriate metallized Mylar that will allow maintaining sensitivity to alpha radiation. Measurements along these lines were made, primarily assessing the attenuation of alpha radiation of the various materials tried. Generally, the glow curve analysis techniques developed somewhat reduced the level of concern over contaminating the sheets, but it is nevertheless prudent for a number of reasons. Firstly, if each sheet is to be used numerous times, a protective, radiation transparent, disposable covering needs to be used in order to prevent contamination of the array by radioactive matter. Secondly, we found that electrostatic discharge into the sheet was of concern because this will give false indications of the presence of radioactive material. An electro-static discharge can induce a thermoluminescent glow curve in phosphor that is entirely equivalent to that induced by alpha, beta or gamma radiation. For this reason, a metallized or conductive package is required in order to dissipate any electrostatic charge near the sheet. Finally, because of light sensitivity of TL phosphor (either a light induced signal or light induced fading of signal may occur), the arrays are stored in black plastic envelopes.

A Mylar film, metallized on both sides, is appropriate for achieving the surface contamination and electrostatic protection goals here. The use of Mylar windows in radiation measurement instrumentation is well known, and need not be discussed in detail. In this project, Mylar films were tested, but an absolute final Mylar package for the sheets was not developed, as this was deferred while solving other problems. For the moment, the arrays are stored in a large electrostatic protection bag of the type used for protecting electronic circuit boards. 


\subsection{Developmental Testing}

During the course of this project, testing of incremental technical improvements and new capabilities was intensively performed. Specific results showing performance of the finalized improvements are presented in this section. Also, we will present here some results of preliminary field tests that were performed during the developmental process, before the system as a whole had all improvements and developments incorporated. Final testing and results are described in a later section.

In order to perform the field tests originally proposed, International Sensor Technology had entered into a number of formal and informal agreements with other groups. A Cooperative Research and Development Agreement (CRADA) was negotiated with Oak Ridge National Laboratory for the purpose of studying applications to specific problems at that site. Informal agreements were entered into with groups at Battelle Pacific Northwest Laboratories and EG\&G Energy Measurements, Santa Barbara, CA. The work with EG\&G concerned soil contamination measurements at the Nevada Test Site. Due to the delays caused by the array materials problems, these tests could not be performed with the fully improved system before these agreements were significantly impacted by DOE program cuts. All three of these agreements were affected by reassignment, retirement or termination of group members. No significant work was performed within the CRADA. One significant test on improved arrays was performed with the EG\&G group. A graduate student wrote a Master of Science thesis using the results from a test performed at Battelle with the silicone based arrays (Oldfield). Some of the preliminary field test work was performed with silicone arrays when it was realized that the NRI-TL could be controlled by manually operating the ventilation system.

Most on-site exposures for development testing were performed with an alpha radiation source consisting of a quantity of ${ }^{238} \mathrm{U}$ deposited on an $8 \mathrm{~mm}$ diameter disk. This source had a calibrated activity of 466 disintegrations per minute (dpm). Additional sources on site were an uncalibrated ${ }^{241} \mathrm{Am}$ alpha source and a $20 \mathrm{~mm}$ diameter, 9.72 microcurie ${ }^{99} \mathrm{Tc}$ beta source. The principal focus was on alpha detection.

\subsubsection{Improvement In Measurement Sensitivity and Background Suppression}

Figure 18 is shown to give a qualitative impression of the overall improvements in light measurement sensitivity and suppression of NRI-TL. The sheet here was of a continuous, polyimide/phosphor layer. Comparison of this figure with Fig. 7, obtained during Phase I on a silicone/phosphor, discrete spot array, shows a vast improvement in sensitivity. In Fig. 7, the minimum exposure interval to the Uranium test source is 5 minutes. In Fig. 18, the one, two, and three minute exposures are easily discernible. Further, in Fig. 7, a second reading of the array was performed and subtracted from the first, whereas in the present figure, no manipulation of the background has been performed. These results are not directly comparable in terms of signal levels because of the change in the photomultiplierand associated circuitry.

The relative levels of signal from array pixels are 18.5 units per incident alpha particle compared to the inherent background of about 8 units per pixel, reading an array one hour after annealing. The relative signal emitted by a pixel given ${ }^{137} \mathrm{Cs}$ gamma radiation (without equilibration build-up material) is 163 units per $\mathrm{mR}$. At the end of Phase I, the best performance of signal-to-background for alpha detection was 6 alpha particles incident on a pixel yielding a signal equivalent to the background level (in these terms the current signal-to-background performance is approximately 0.43 alpha equivalent background compared to 6 alpha equivalent). The improvement in signal-to-background performance amounts to a factor of approximately 14. This improvement is the aggregate effect of the enhancements to the signal acquisition and processing systems and the improvements in array fabrication. Note that obtaining the figure quoted for Phase I results required subtraction of two readings, whereas the comparative value for the present effort is obtained with a single reading. 


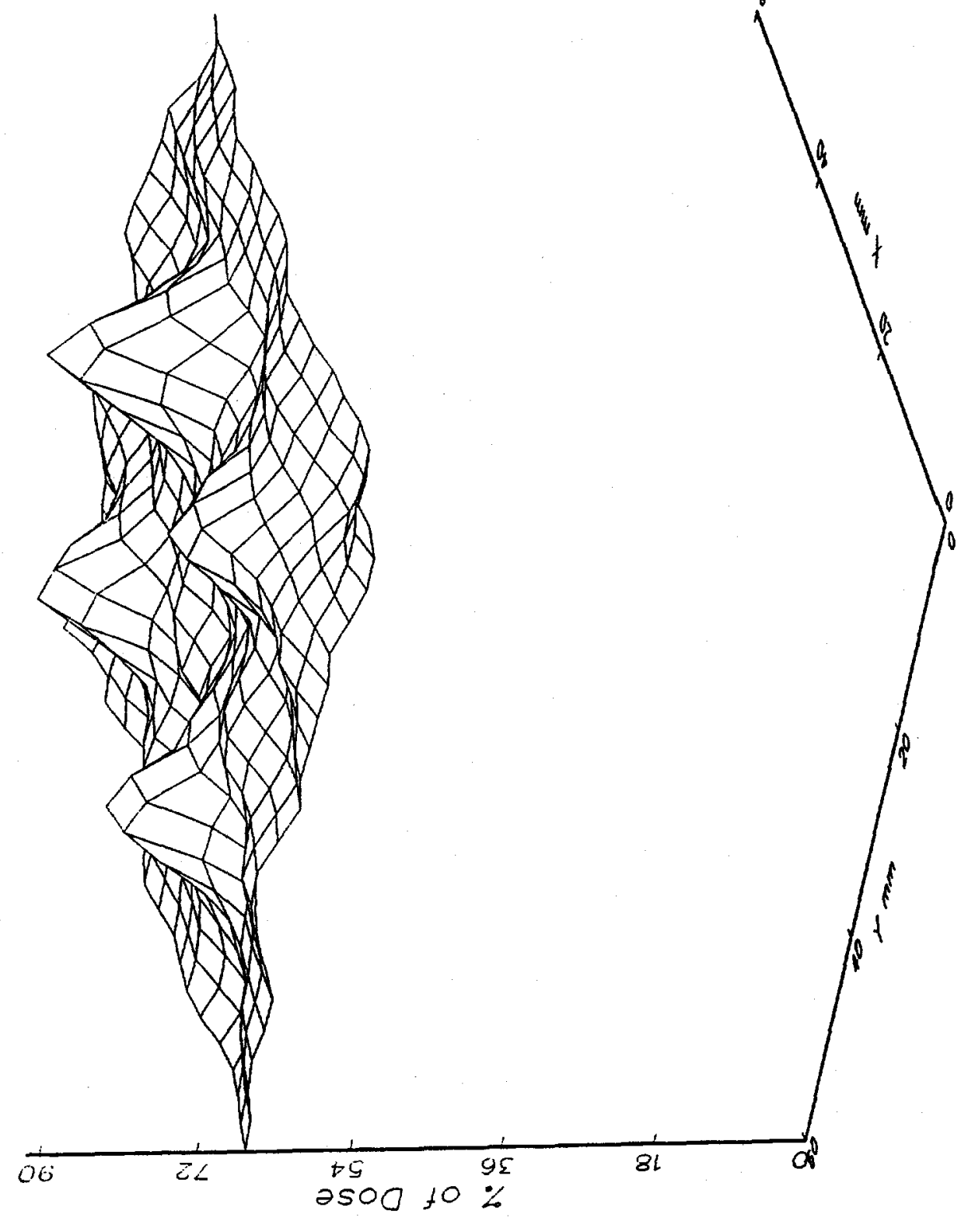

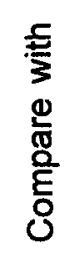

ญํํㄱ

똥

공

㠃

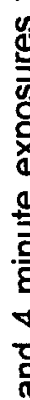

m

N

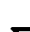

焉

Ð

忞

흥

음

象

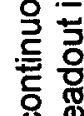

8

次交

芌

$\stackrel{\infty}{-}$

훈 


\subsubsection{Mylar Tests}

Various metallized Mylar films were tested on the arrays for attenuation of alpha radiation using ${ }^{238} \mathrm{U}$ and ${ }^{241} \mathrm{Am}$ sources. Mylar thickness of $0.22 \mathrm{mg} / \mathrm{cm}^{2}$ attenuated the ${ }^{241} \mathrm{Am}$ signal by approximately $11 \%$, and $1.16 \mathrm{mg} / \mathrm{cm}^{2}$ Mylar attenuated it by about $32 \%$. In comparison, $0.85 \mathrm{mg} / \mathrm{cm}^{2}$ Mylar attenuated ${ }^{238} \mathrm{U}$ alpha particles by $43 \%$. The $0.22 \mathrm{mg} / \mathrm{cm}^{2}$ film attenuated the ${ }^{238} \mathrm{U}$ alpha particles by $17 \%$. This thinner material is generally too delicate for applications where abrasion would be significant. These two isotopes cover the general range of emitted alpha energies of interest. For surface contaminant measurement of ${ }^{238} \mathrm{U}$ the use of the more rugged, thicker film implies that a doubling of array exposure time would be required.

\subsubsection{Application of Software Routines}

Before proceeding, it is useful to show the effect of applying the convolutionalgorithms discussed above to array readings. Figure 19 shows a contour plot of an array reading after various exposures to the ${ }^{238} \mathrm{U}$ source. Given the black-and-white format, considerable thought is required to interpret these plots. In order to analyze these, generally the outermost contour has the smallest value listed to the right of the figure and works inward. Only values listed at the right are displayed in the figure. Thus, the density of contour lines is indicative of the enclosed signal intensity. In this figure, two of the three 2 minute exposures are detected.

Figure 20 shows the result of processing the raw data with a $33 \times 33$ convolution kernel, with the output displayed on the same size axes as the input data. Given the $3 \mathrm{~mm}$ array grid spacing, this kernel represents a square detector window of $98 \mathrm{~mm}$ width, for an area of $96 \mathrm{~cm}^{2}$. The pronounced square contours represent the totality of window center positions which would enclose the three 32-minute exposures. Any one contour value represents a curve which, if traced by the center point of a detector window, would yield a constant signal level. This is the nature of the output of these convolution-processed data sets. Recall that only a central area of this figure represents valid data, as the convolution routine uses zero padding along the edges of the input array.

Figure 21 shows the result of applying a smaller kernel, here an $11 \times 11$ window representing approximately $10 \mathrm{~cm}^{2}$ detector window area. Note that smaller windows make the exposure features more pronounced, as one would expect, and that all three 2 minute exposures are now evident.

An application of the convolution routine in extracting numerical data from a reading is illustrated in Figures 22,23 and 24 . The first of these shows an array reading following ${ }^{238} \mathrm{U}$ alpha exposures of 10,20 , and 30 minutes duration. Fig. 23 shows the resultant output of applying a $33 \times 33$ kernel $\left(100 \mathrm{~cm}^{2}\right)$ to this data and Fig. 24 shows the same input data processed with a $3 \times 3$ kernel (the smallest square kernel that can enclose the region exposed by the $8 \mathrm{~mm}$ source). Using customized MATLAB routines, data from these processed arrays can be extracted. In Fig. 25, we plot the maximum values within the exposure areas from the previous three figures. Note that the raw data can be considered as a convolution with a $1 \times 1$ kernel. Generally, the behavior demonstrated in Fig. 25 is as expected. The larger y-intercepts for larger kernels are due to the greater number of unirradiated pixels contributing background to the signal enclosed within the kernel.

Note that in this example the derived signal values (which are in arbitrary units) correlate linearly with exposure time, i.e., the total number of disintegration so the source during the exposure interval. However, systematic variations in the sheet sensitivity have not been normalized away. The curve fits for the $3 \times 3$ and $33 \times 33$ convoluted data sets in Fig. 25 yield an approximate value of 4 signal units per source disintegration. The fact that these two convolution kernels yield similar slopes despite their large difference in window size 


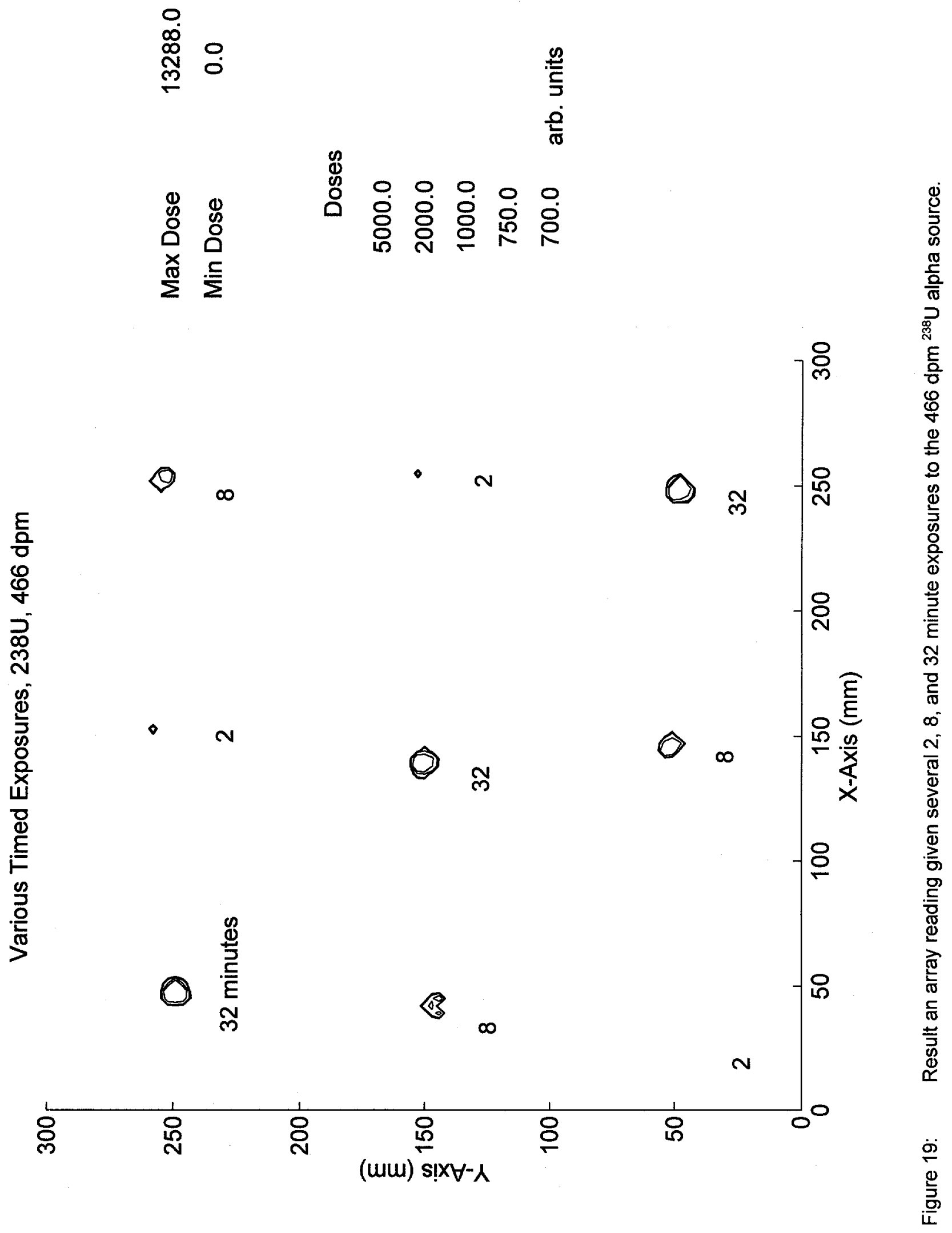


毫誉

高

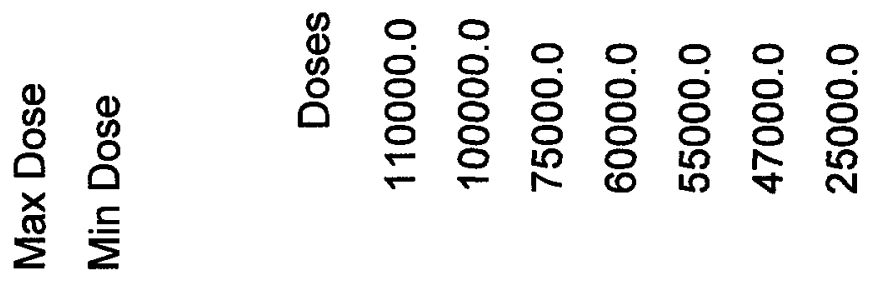

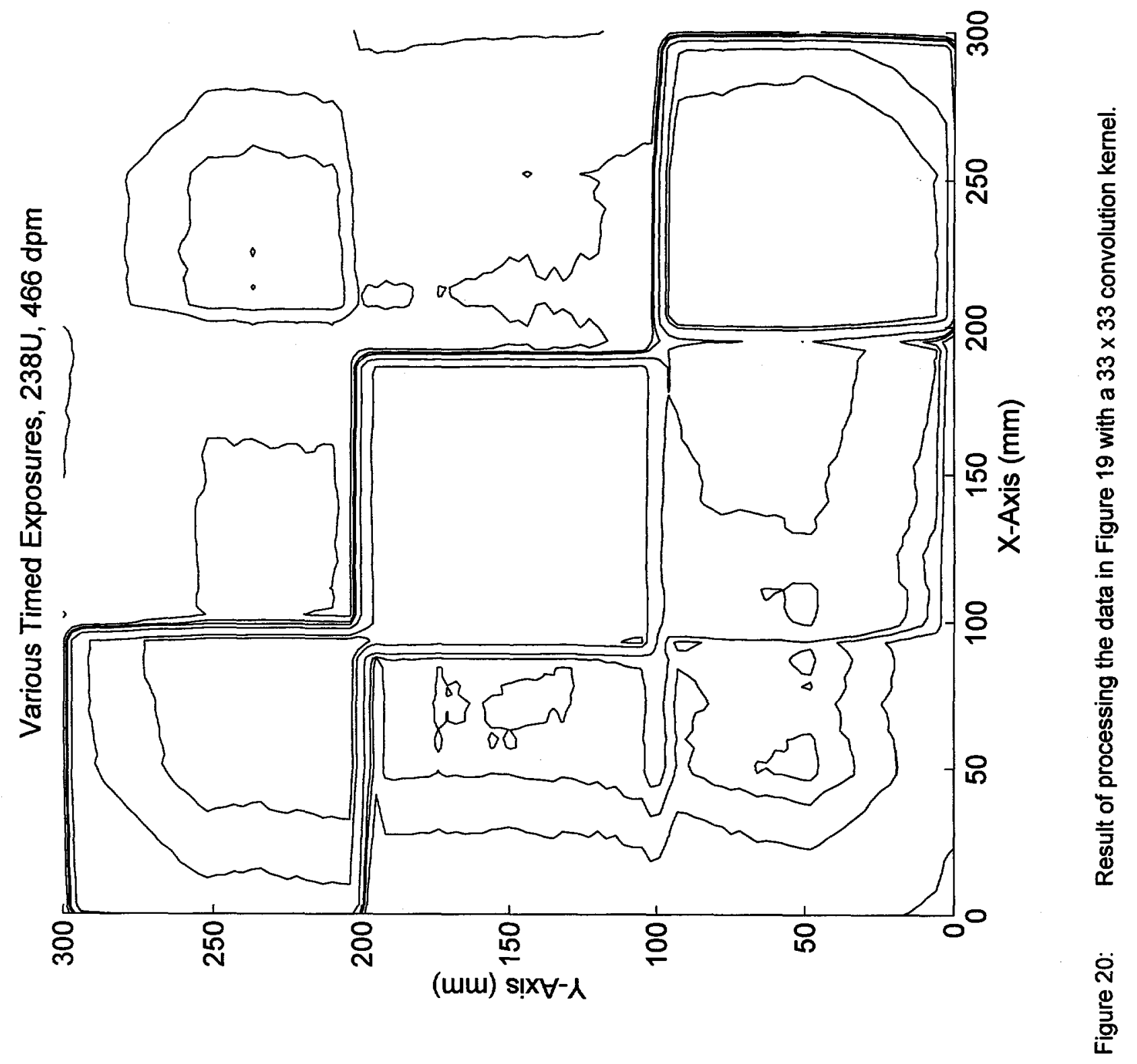




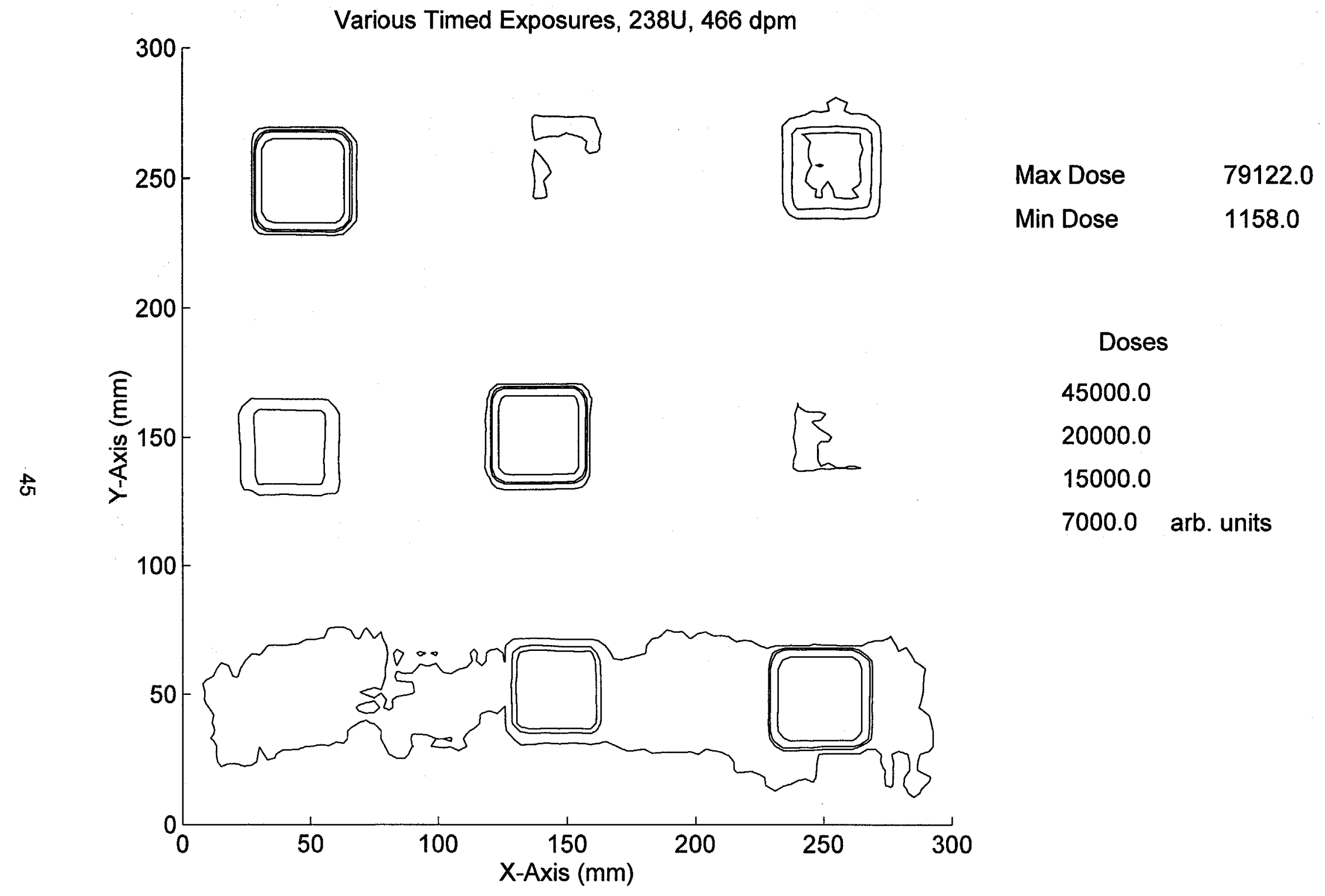

Figure 21: Result of processing the data in Figure 19 with a $11 \times 11$ convolution kernel. 


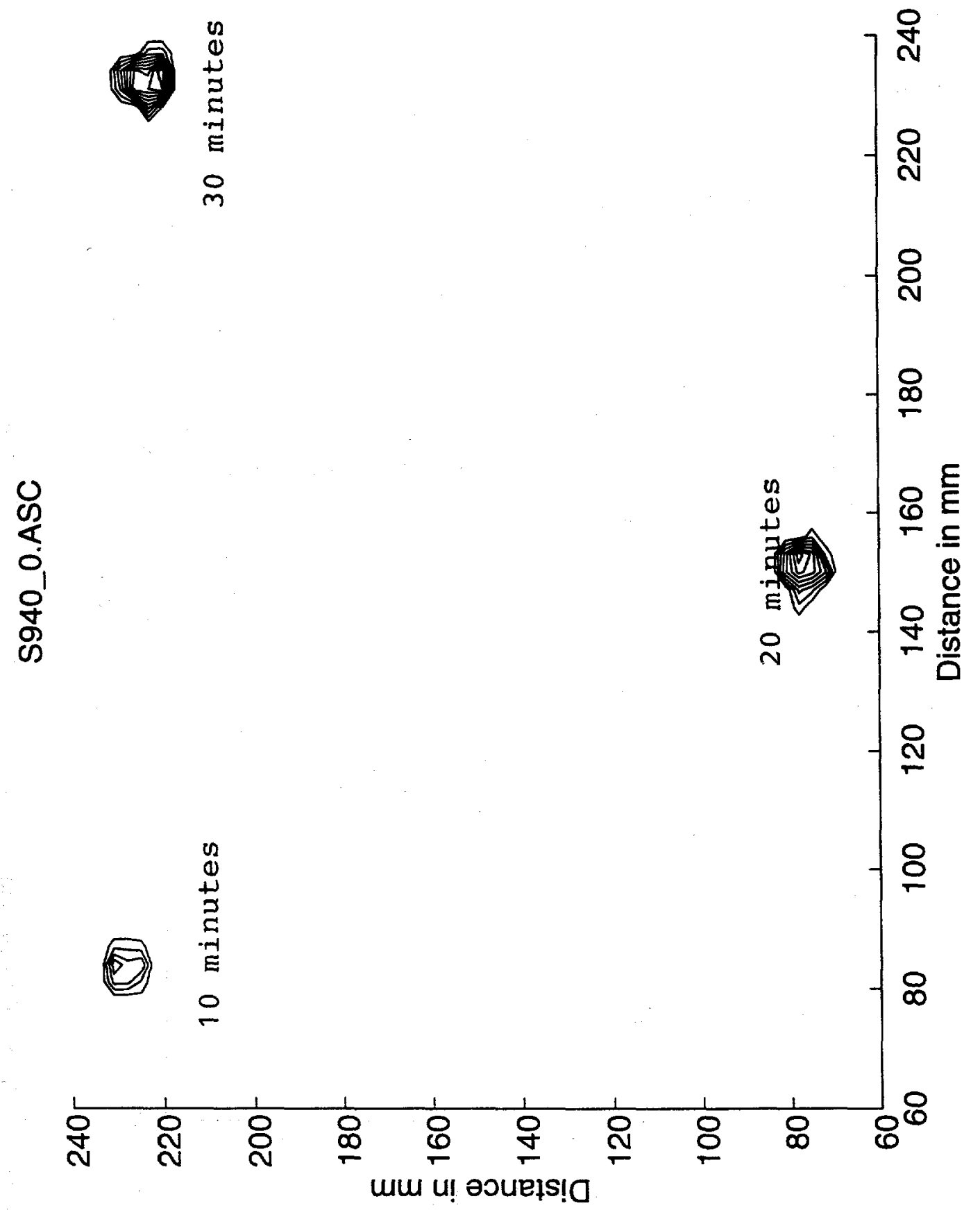

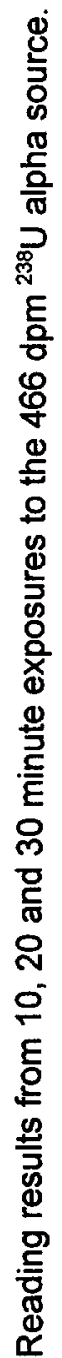

Ṅ
ơ
믄 

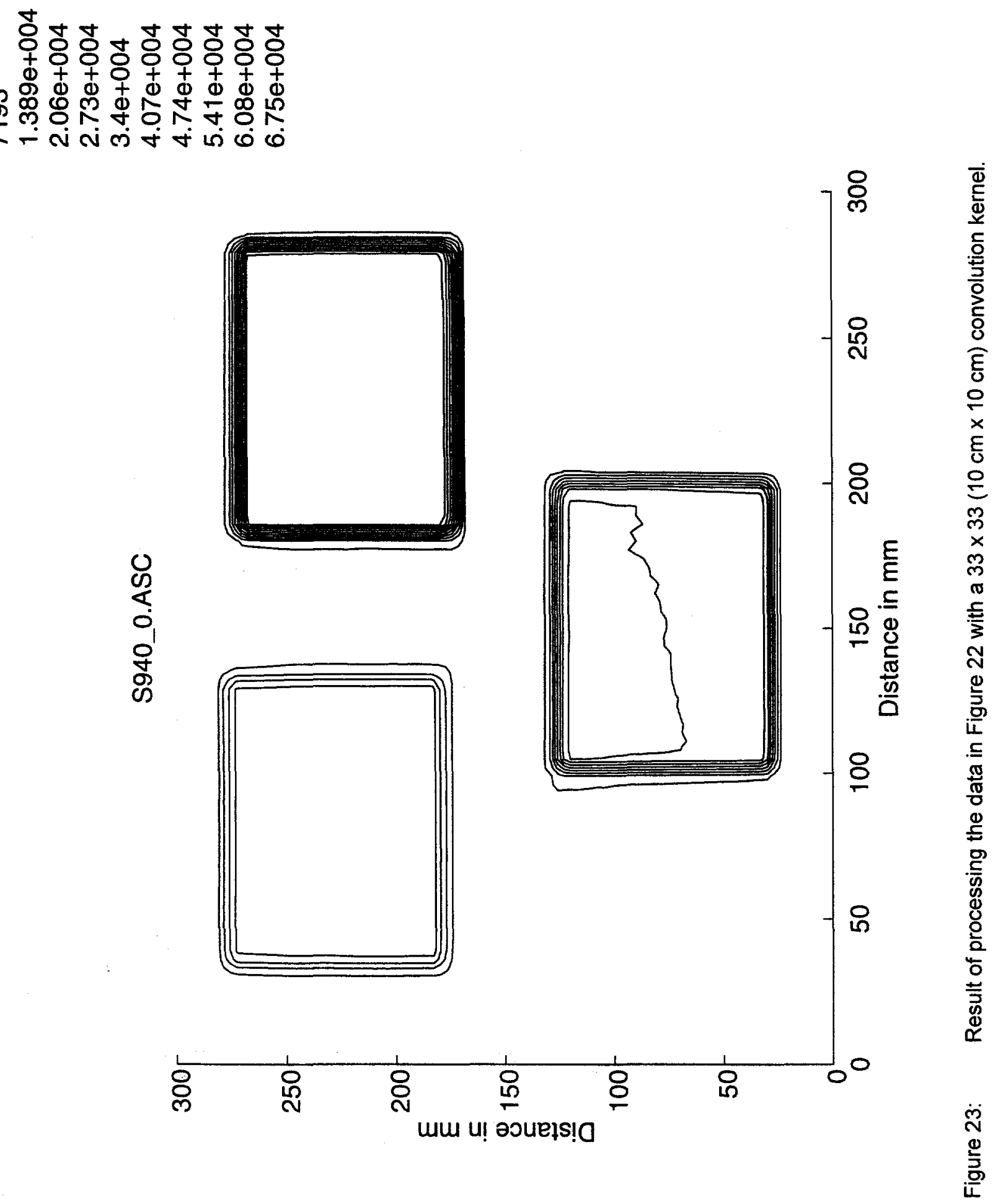


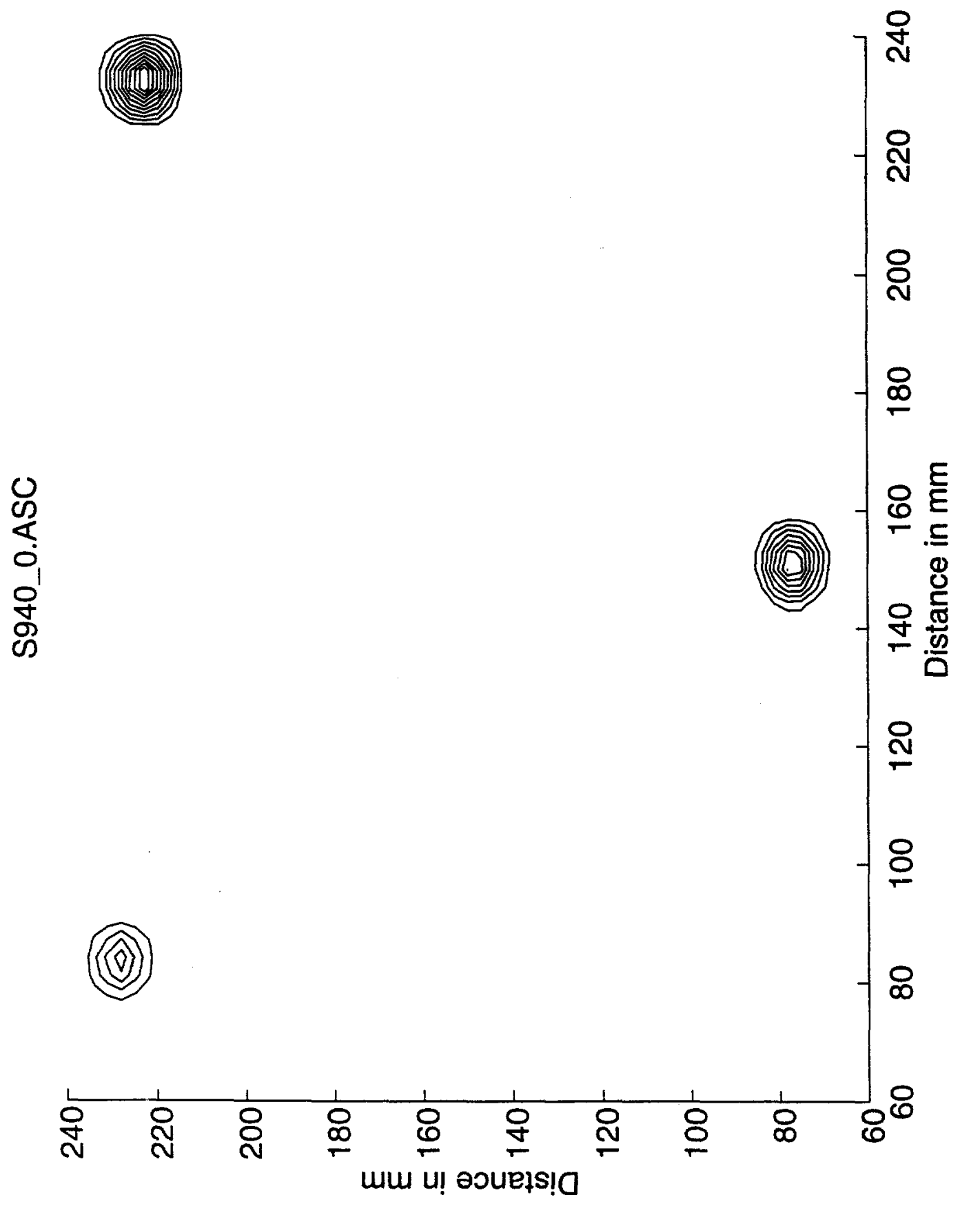

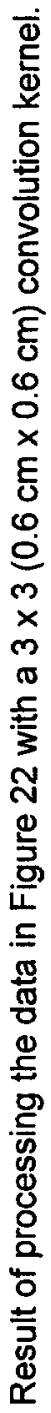

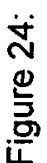




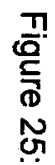

Max value of convoluted reading arb. units)

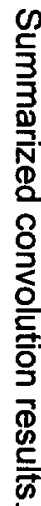

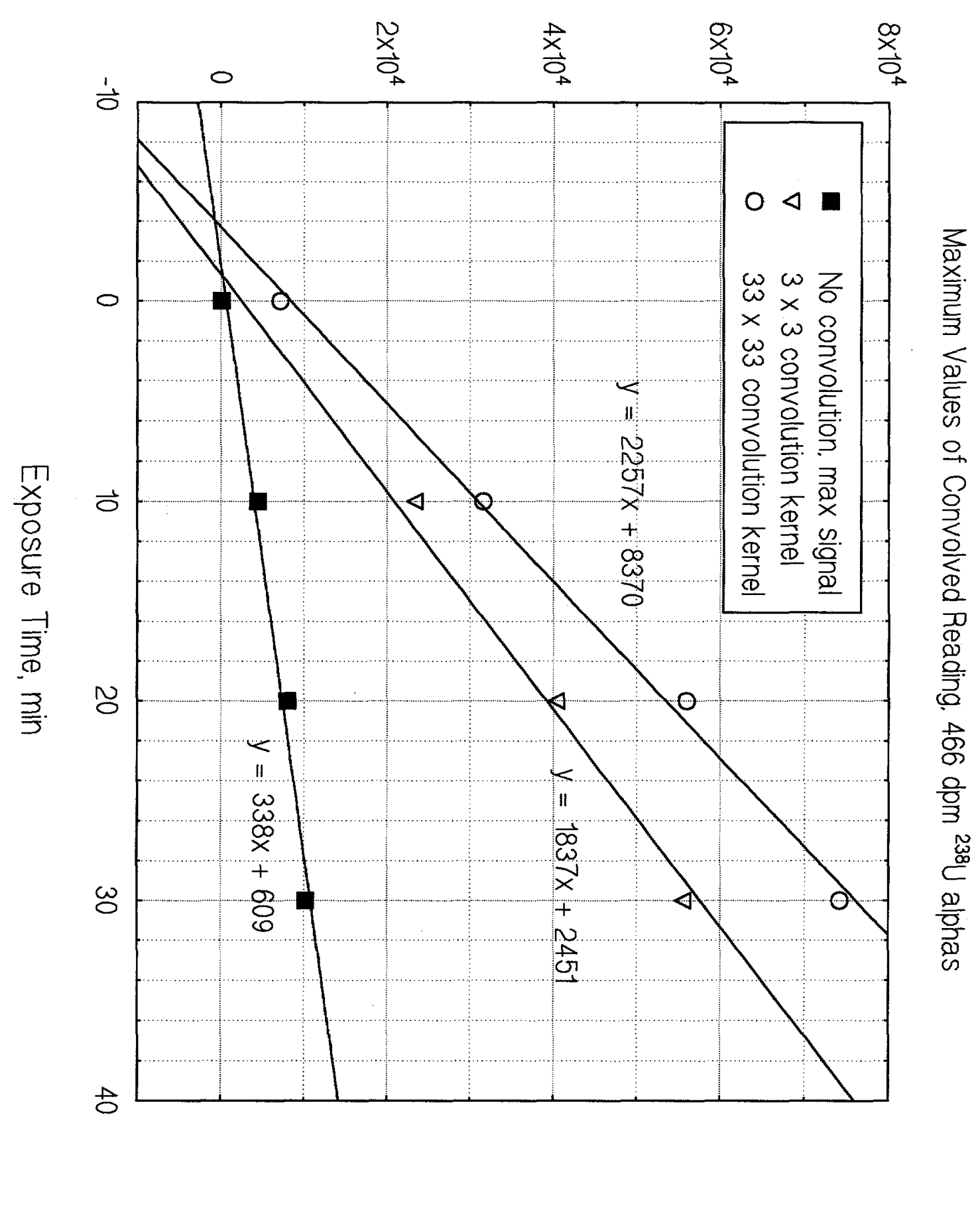


reflects the fact that the entire source is contained within both of these windows and is the major contributor to the signal in both cases.

An additional example of using the convolution routine for source detection is shown in Figure 26 . In this case, the sheet was exposed to the ${ }^{238} \mathrm{U}$ alpha source for various time intervals. All exposures were detected using the smaller $5 \times 5$ convolution kernel, but one spurious hot spot was also detected, yielding a signal approximately equal to that induced by a 2-minute exposure. The purpose of this figure is to demonstrate that zones of lower level activity can be detected more easily with small convolution windows, but there is increased probability of false positive readings.

For interest, we present here two measurements obtained from exposures at the Nevada Test Site. These examples were the last measured from this location, but the only ones with fully improved arrays. Figure 27 shows a contour plot of soil surface activity at a location known to be contaminated with ${ }^{241} \mathrm{Am}$. Figure 28 shows a contour plot of a soil activity depth profile. In this experiment a bare array was vertically inserted into a slit in the ground created by a special shovel. Five centimeters of the sheet remained above ground, while twenty centimeters were in contact with soil below the surface. This array had a $5 \mathrm{~cm}$ portion trimmed away for another experiment. This direct soil insertion proved the value of the glow curve analysis technique in evaluation of the sheet, and hints that covering arrays to protect from soil and abrasion is not as mandatory as might be expected. Fig. 29 shows an application of a specialized convolution kernel developed to derive an average soil activity profile. Here, a $1 \times 101 \mathrm{kernel}$ was applied to the input data such that all pixels in a row parallel to the soil surface are summed to give an average activity at depth. This result, while not calibrated for soil activity level, nevertheless is in general agreement with the known contamination characteristics and remediation attempt history at this location. ${ }^{9}$ The top 5 centimeters of clean soil had been placed to prevent spread of the radioactive material. The example here is indicative of potential cost-saving applications of this technology.

In order to demonstrate the utility of the coarse/fine array scanning routine, an experiment was performed to test its ability to detect surface activity of various distributions. For a given fixed contamination level within a $100 \mathrm{~cm}^{2}$ area, it was anticipated that a uniform distribution would be the most difficult to detect. However, obtaining an exposure to a certainly uniform activity distribution from a planar radioactive source was not feasible. Therefore, a technique was developed to scan the $8 \mathrm{~mm}$ diameter ${ }^{238} \mathrm{U}$ alpha source over a fixed area in a manner such that all array pixels had equal exposure time under identical source segments. The scanning optics head in another two-dimensional laser TLD array reader was slightly modified and the $466 \mathrm{dpm}{ }^{238} \mathrm{U}$ alpha source was placed in the bottom of the head. With this setup, the alpha source could be scanned across the sheet to give repeatable exposures in both time and placement. The source was about $0.75 \mathrm{~mm}$ above the sheet. The sheet area was conceptually divided up into nine equal square fields of $100 \mathrm{~cm}^{2}$ area. An algorithm was developed to give an exposure to each of the four corner squares. Each of the four corners were given a different exposure distribution, but the total time for each was 30 minutes, yielding a constant $466 \mathrm{dpm} / 100 \mathrm{~cm}^{2}$ for each exposed field. For the results shown in the following figures, the exposures were as follows:

$$
\begin{aligned}
& \text { Upper right field } \\
& \text { Bottom right field } \\
& \text { Top left field } \\
& \text { Bottom left field }
\end{aligned}
$$

One $30 \mathrm{~min}$. exposure.

Four $7 \mathrm{~min} .30 \mathrm{sec}$. exposures

Sixteen $1 \mathrm{~min} .53 \mathrm{sec}$. exposures

Uniform coverage of field, $30 \mathrm{~min}$.

The uniform exposure in the bottom left field was accomplished by slowly scanning the alpha source centered on element rows and columns at a speed which completed the scan in 30 minutes. The diameter of the source circumscribed three rows of pixels when centered on a row. Since the source was

${ }^{9}$ Personal Communication, W. Quam. 


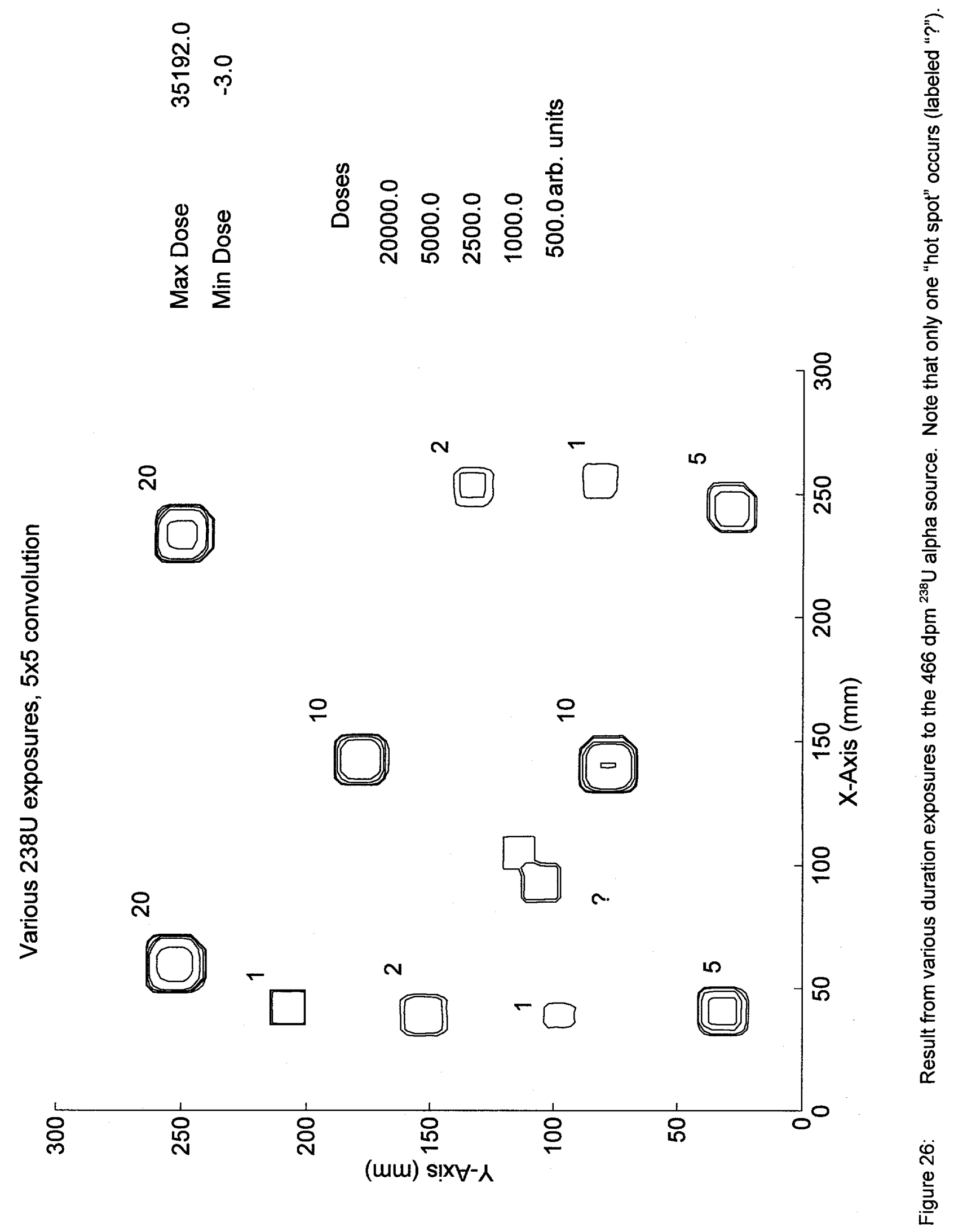




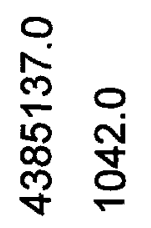

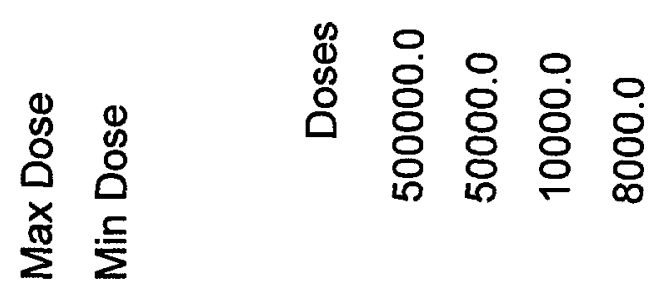

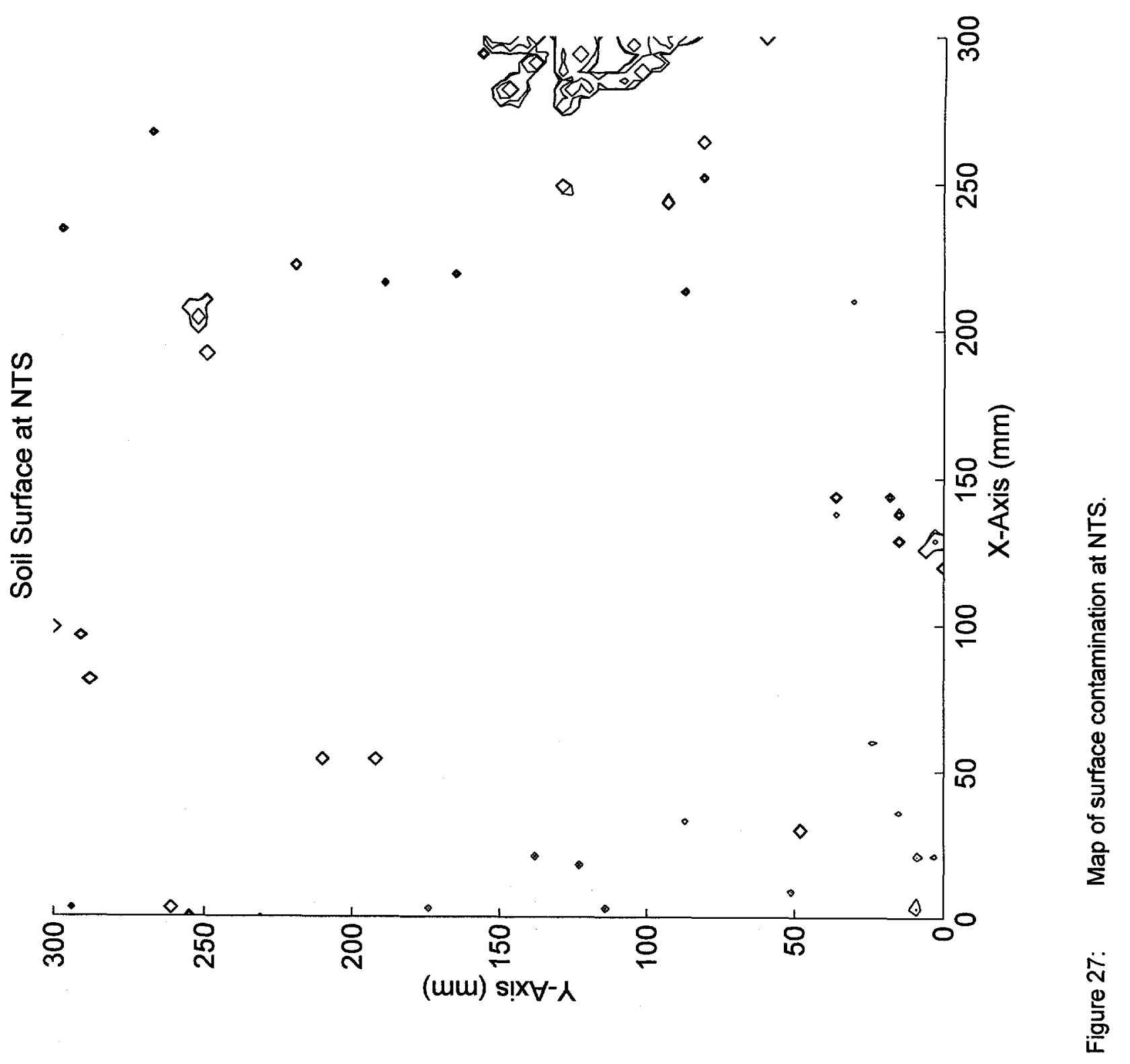




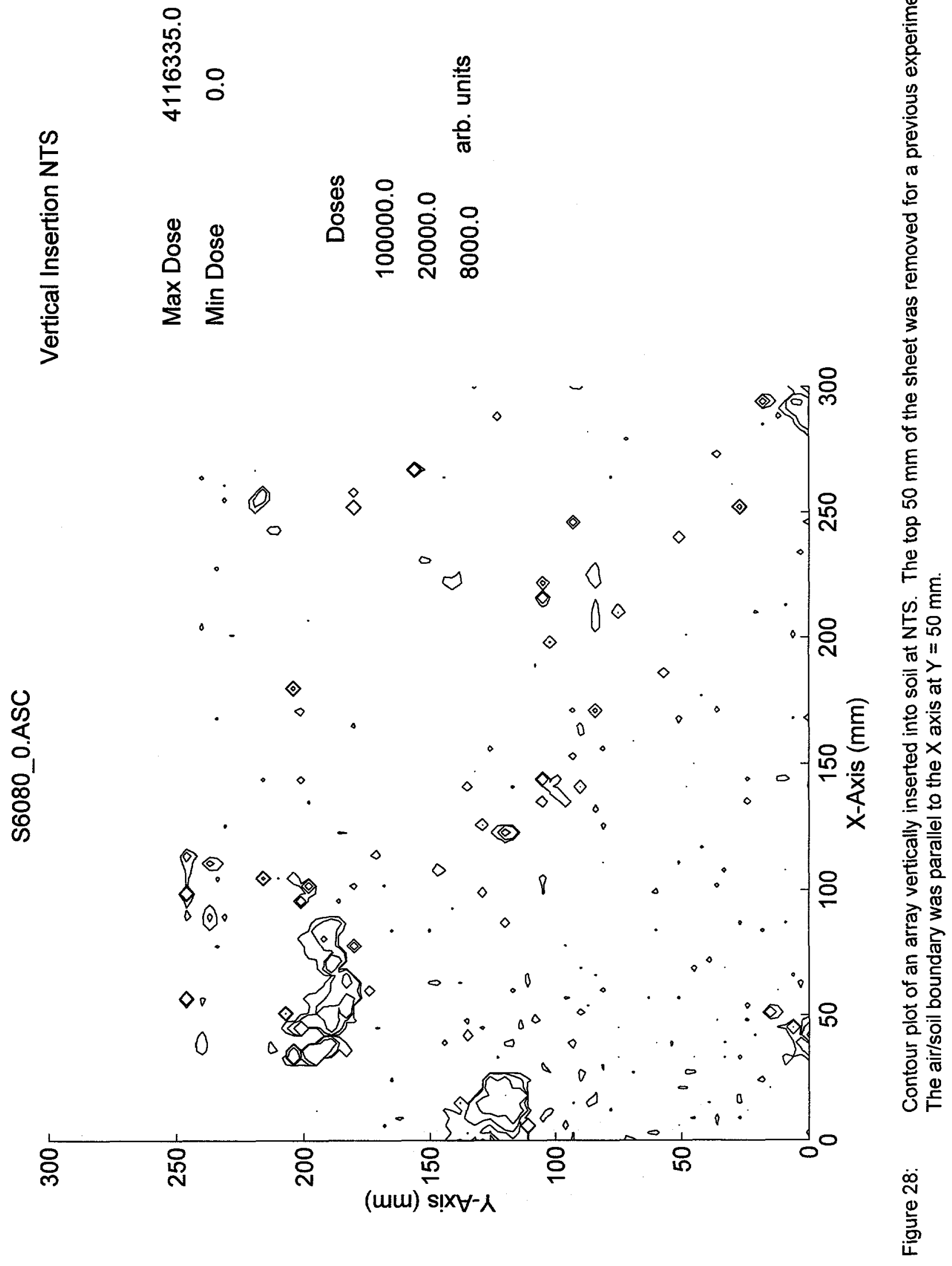




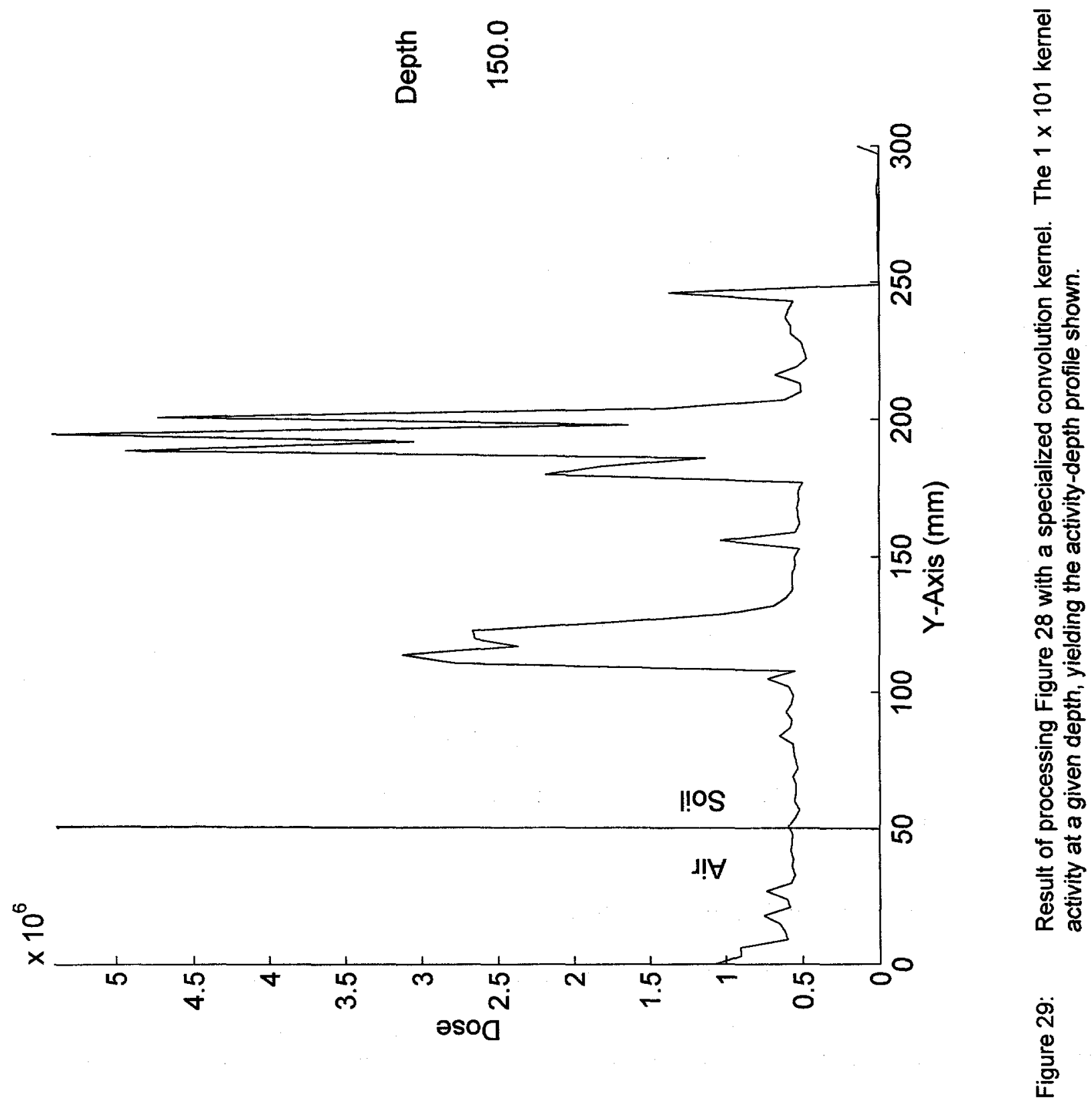


jogged only a single row before scanning the next row, this exposure technique probably produces a more uniform exposure than that obtainable from a large area source of plated material. These exposures span the gamut from uniform to very localized. If this exposure represented the contamination distribution of a real surface that was scanned with an active detector of $100 \mathrm{~cm}^{2}$ area, each of these fields would in principle yield identical measurement results. We examined our results in raw form, and in a form where a $100 \mathrm{~cm}^{2}$ square kernel was convoluted with the measured data to determine whether these distributions are detectable under comparable conditions.

A total of eight sheets were exposed and read out. Five were read out with the intelligent coarse/fine scanning routine, and three were read at resolution 1 (straightforward reading of every spot). Our goal was to compare the detection capability of the two techniques. Figures 30 and 31 show example contour plots of raw data for the coarse/fine and full resolution readings. The single 30 minute exposure and the four $7 \mathrm{~min}$. $30 \mathrm{sec}$. exposures were easily detected on every reading. The field with the sixteen $1 \mathrm{~min} .53 \mathrm{sec}$. exposures normally detected between 10 and 12 of the exposures in raw form. In direct comparison between the resolution 1 and the intelligent readout routines, there is no unequivocal preference for the slower, methodical approach based on these results because neither example shows convincing evidence of activity in this field. In the lower left, uniform exposure field, the contour plots show more signal in the Resolution 1 readings, but there is apparent signal with the intelligent readout, too.

Figures 32 and 33 show these data convoluted with the $100 \mathrm{~cm}^{2}$ kernel. Basically the same conclusions hold, but the detection of the uniform field is much more certain in this case, especially in Fig. 33 . These plots are not the best format for viewing this data, however. Additional signal processing can bring this out more clearly, as can pseudocolor plots. Nevertheless, we can conclude that the faster, intelligent readout technique is effective in detection of activity, and the increased rate of readout may make an increase in sheet exposure time worthwhile.

An additional test of the coarse/fine scan routine was performed for short exposures. This test consisted of spot placement of the $8 \mathrm{~mm}$ diameter, $466 \mathrm{dpm}{ }^{238} \mathrm{U}$ alpha source. Two 3 minute exposures and eight 2 minute exposures were given to each of five full size sheets, for a total of fifty exposures. Thirty six out of the fifty exposures were detected. All ten of the 3 minute exposures were easily detected, and twenty six out of forty 2 minute exposures were detected. Non-detection of some of the 2 minute exposures may be attributed to either placement of the source between reading positions, or the nonuniformity of the phosphor layer in some regions of the sheet. Two more sheets were exposed to a similar pattern except that all ten exposures were 3 minutes. Nineteen out of the twenty exposures were detected. The size of this source is such that, if randomly placed on a sheet, only one, but at most two, pixels that would be read in a coarse scan would be irradiated at all. Since the source is not uniform, and we are trying to determine the detection threshold, it is not surprising that some of the weakly irradiated pixels are in fact missed. The implication is, at least for a patch of contamination of this extent $(8 \mathrm{~mm})$, it requires an exposure time of at least 3 minutes, or 1400 disintegrations, to detect a nominal $500 \mathrm{dpm}$ activity.

This section has provided a summary of testing activities performed during the development stages of this project. In the next section, final testing and calibration with all incorporated improvements is detailed.

\subsection{Final Testing and Determination of Detection Limits}

The final tasks performed in this project were to obtain expert input into how this system might be used, development of an appropriate fielding protocol, and to calibrate the system using large area planar sources of alpha and beta emitting isotopes. Using this data and advice, we derived a protocol that defines required array exposure intervals for measurement of surface contamination at the release guideline limits 
$\begin{array}{ll}0 \\ \infty \\ \infty & 0 \\ \infty & 1\end{array}$

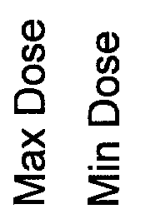

क $\frac{2}{2}$

○

(0)
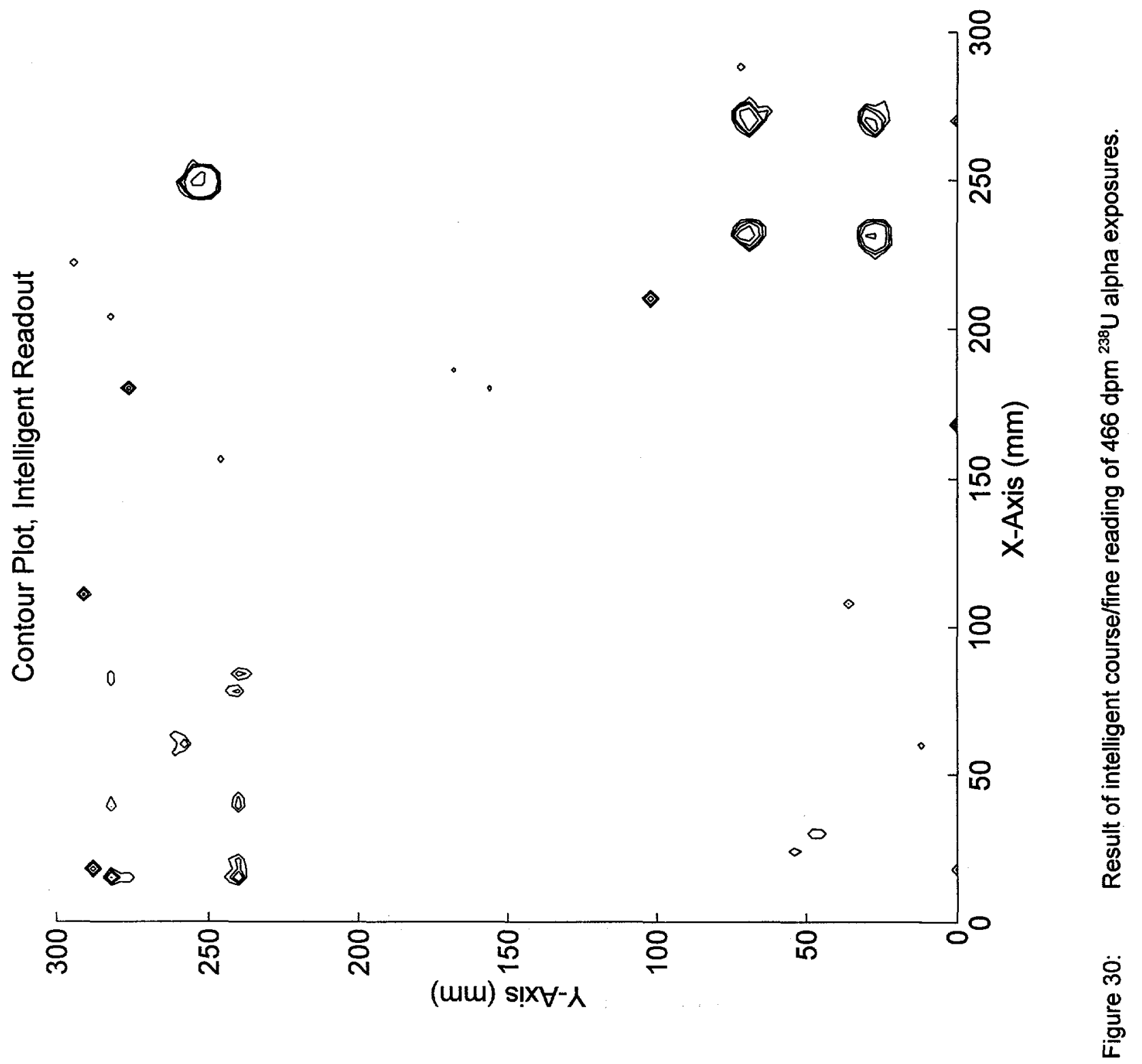

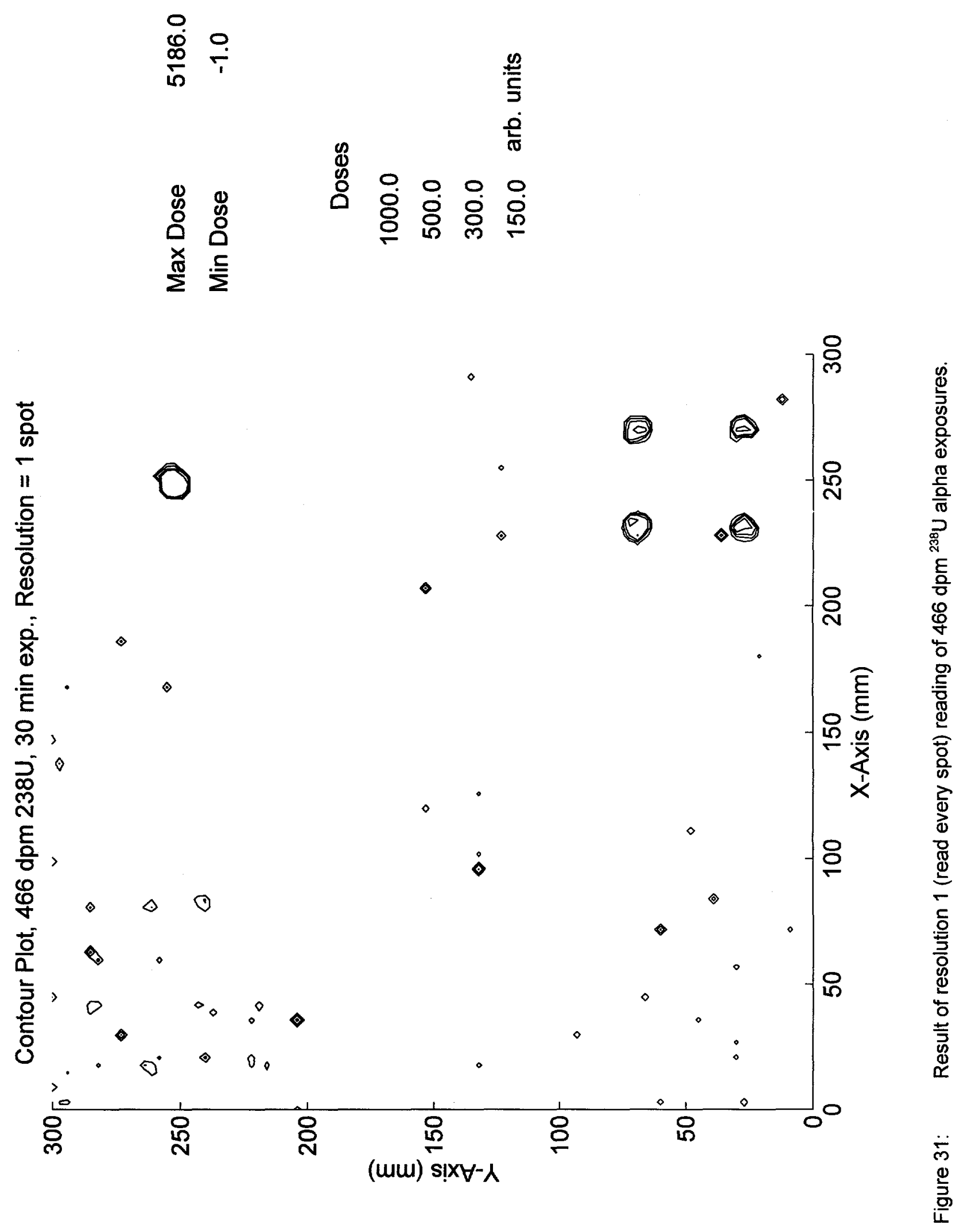
$\begin{array}{ll}0 & 0 \\ \infty & 0 \\ \infty & \infty \\ m & 0 \\ 0 & 0 \\ 0 & \infty\end{array}$

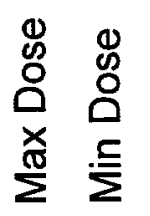

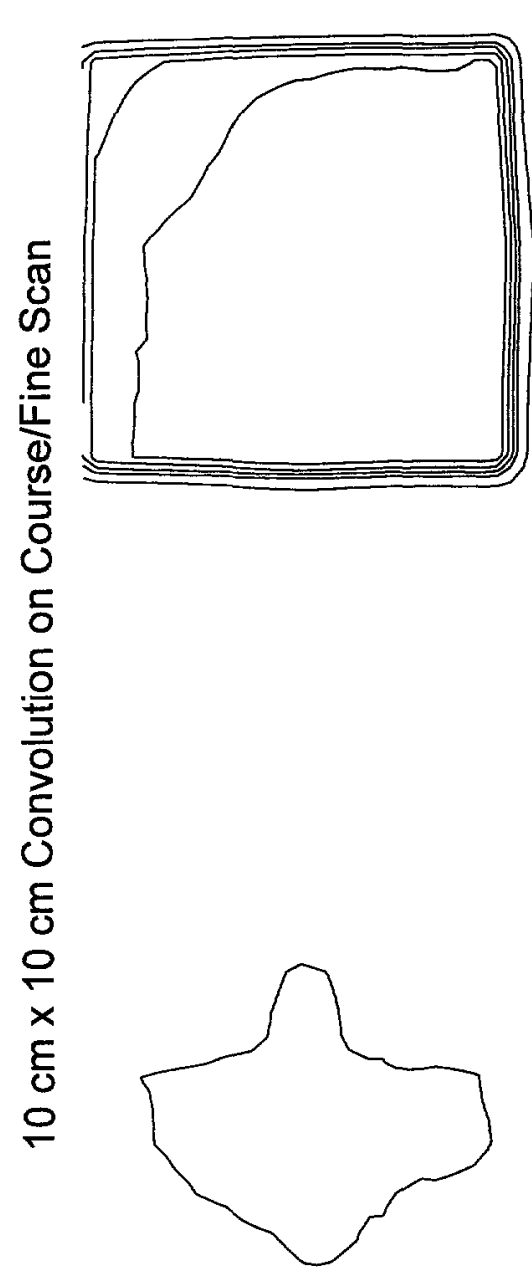

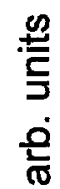

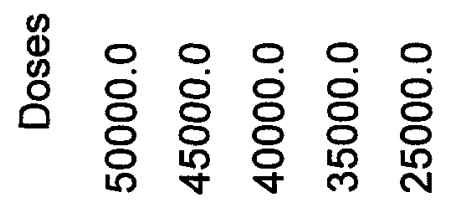

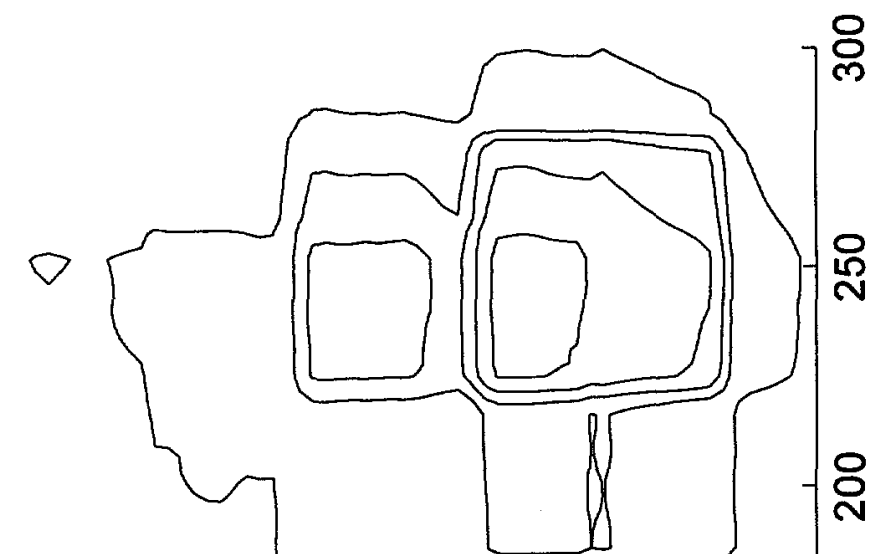

윰

윰 $\frac{\substack{\xi \\ \frac{1}{x}}}{x}$

음

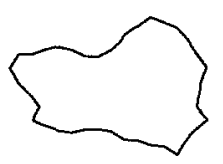

유

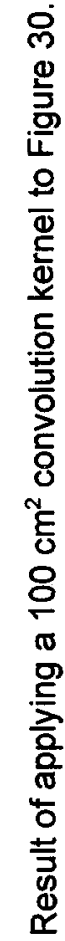

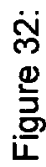




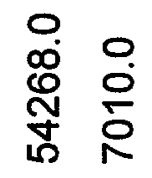

$\stackrel{\infty}{5}$
$\frac{5}{5}$
은

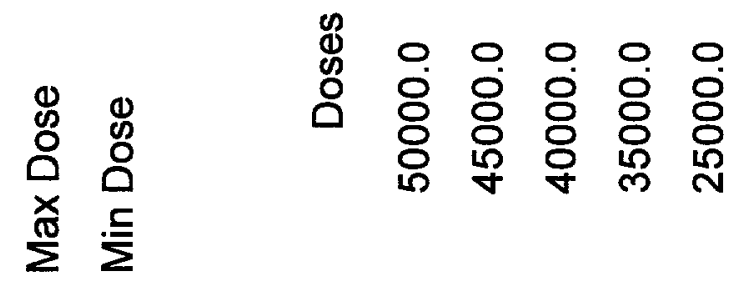

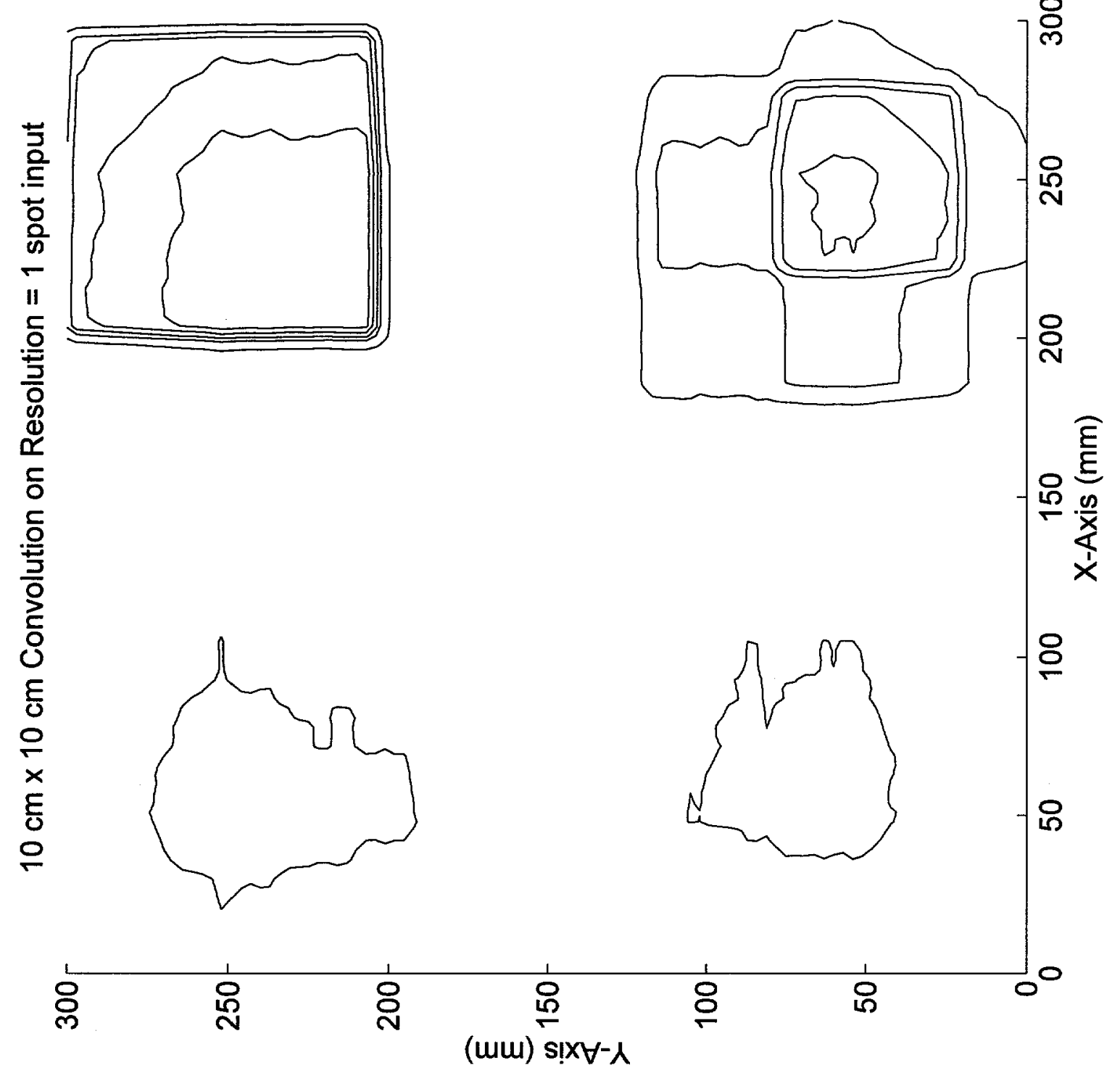

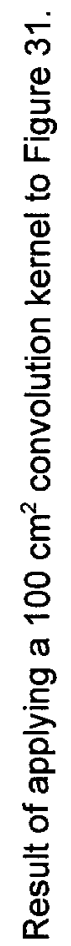

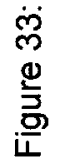


listed in Table I. This should only be considered as a first determination of the required exposure times, as further developments and testing may cause these values to be changed. Only a few isotopes were used in these measurements, and application of these results to radiation of significantly different character (primarily beta radiation of much lower particle energy than reported here) cannot be considered valid.

\subsubsection{Recommendations}

An information interview was conducted with Michelle L. Johnson, Sr. Research Scientist in Radcon Instrumentation Services at Battelle Pacific Northwest Laboratory, located on the Hanford Reservation in Washington State. Her expertise in the contamination survey field derives from her capacity in providing qualified instrumentation, and participating in approval of survey protocols, for all contractors at the Hanford site. Instrument and protocol qualification is based on the instrumentation's and the methodology's capability of measuring contamination at the prescribed limits.

For our purposes here, we can summarize the advice on the basic requirements the system should meet (based on the release limits). Michelle Johnson's recommendation was to define the capability of the system to measure the average alpha contamination at the $100 \mathrm{dpm} / 100 \mathrm{~cm}^{2}$ level, and to make certain that there was the capability of measuring, with no more than $5 \%$ false negative readings, the maximum allowed level of $300 \mathrm{dpm} / 100 \mathrm{~cm}^{2}$ occurring once in a square meter. It is of paramount importance to evaluate the minimum contact time required to measure with statistical confidence the desired release limit contamination levels. The primary goal should be to use the system as a "go/no-go" gauge for detection of contamination, (i.e., is the surface activity above or below the limit), in the defined guideline units, $\mathrm{dpm} / 100 \mathrm{~cm}^{2}$. Secondary to this goal would be obtaining an actual value for the contamination surface density.

\subsubsection{Detection Criteria}

The widely cited work of Currie (Currie, 1984) was used for guidance in developing the proper measurements required to meet the release criteria at the appropriate level of statistical confidence. Following Meyer, et al, we have adapted the work of Currie to the case of passive, integrating detectors, where the more appropriate variable is detector response to total number of nuclear disintegrations over a measured time interval rather than count rate (Meyer, 1994). MARSSIM provides a review of the pertinent material.

Sheet exposures to large area alpha- and beta-emitting sources were performed for calibration and test purposes. These exposures were obtained from calibrated sources maintained by Battelle Pacific Northwest Laboratory. A large number of background readings were performed, as the limits of detection are strongly governed by background readings.

Following the analysis of Currie, we denote $\sigma_{0}$ as the standard deviation of the background measurement, where no radiation (other than background) has been incident on the sheet. The critical signal, $\mathrm{S}_{\mathrm{c}}$ is the value of net signal which has a statistically significant level above the mean background. Here, statistically significant means that some chosen level of signal is sufficiently above the background level that it will be accepted as a positive, i.e., greater than background, result. The choice of the level for $S_{c}$ depends on the criteria for tolerance of false-positive results, also known as type I error. In radiation measurements, this tolerance is usually taken to be $5 \%$. This translates into a $95 \%$ confidence that a signal of level $S_{c}$ is actually not just a statistical variation of background measurement. The net signal level $\mathrm{S}_{\mathrm{d}}$ is called the detection level, and is the level of signal caused by radioactivity which can be distinguished from $S_{c}$ with a given statistical confidence. If this statistical confidence interval is again $95 \%$ (again, the normal case for radiation measurements), then there is a $95 \%$ probability that an activity of this level will yield a signal greater than $S_{c}$. Therefore, there is also a $5 \%$ probability that an activity at this level will be 
measured as below background. This is known as a false-negative or type 2 error. In other words, the levels are usually chosen so that there is a $5 \%$ chance that a background reading will be greater than $S_{c}$, and a $5 \%$ chance that a reading from a given activity will be less than $S_{c}$. Under certain conditions pertaining to the nature of the measurement distributions, when both confidence intervals are taken as $95 \%$, then

$$
\begin{aligned}
& S_{c}=1.645 \sigma_{0}, \quad \text { and } \\
& S_{d}=3.29 \sigma_{0} .
\end{aligned}
$$

In this project, we have taken this analysis to serve as a model for determination of the method detection limit (MDL) (Meyer, 1994). We have not undertaken an analysis of the fine points of its applicability. Using background measurement data, we shall apply these equations to derive values of $S_{d}$ for various isotopes, in terms of the number of disintegrations occurring within a $100 \mathrm{~cm}^{2}$ area, and finally obtain array exposure times required to measure surface contamination at the release guideline limits.

\subsubsection{Calibration and MDL}

Figure 34 shows the statistical analysis of the results of a background reading after a freshly annealed array was exposed to a clean surface in our laboratory for 60 minutes. The automated glow curve analysis routine was monitored during the reading. It worked very well, never accepting a glow curve that would be rejected by an experienced human judge. At an arbitrary level of 150 signal units for triggering the analysis algorithm, approximately one percent of the glow curves were analyzed. The small number of analyzed curves would scarcely effect the overall average if they were accepted, and a higher level for glow curve discrimination is warranted, speeding up the readout process. The capability is valuable, but should be used to analyze larger level signals that may be due to spurious causes. In the figure, the distribution is plotted after the results were convoluted with a $35 \times 35$ square kernel. The kernel was enlarged to enclose $108 \mathrm{~cm}^{2}$ because the $33 \times 33$ kernel would underestimate the results, enclosing only 96 $\mathrm{cm}^{2}$. Only odd-dimensioned kernels are allowed, as discussed in 3.2.2.4. Therefore, the results show the total integrated signal that would be obtained by placing a square, $108 \mathrm{~cm}^{2}$ detector in contact with the surface in all possible valid locations (within the graininess of the array) for a period of one hour in each location. The mean value of 27,559 (arbitrary) units is taken to be the mean value of the background signal level. This plot does not follow the normal distribution, a fact that will play a role in what follows.

Figure 35 shows a similar result following a 13 hour array exposure to a clean surface. Here, the mean value is slightly higher, at 29,698 units, but the standard deviation is proportionately much higher than in the one-hour test. Clearly, there are also significant numbers of measurements well above the mean. For a normal distribution, the approximate number of observations or occurrences with values more than 2 standard deviations above the mean should be less than $5 \%$. In the present case, more than $5 \%$ of observations exceed this 2 standard deviation threshold, and the conditions leading to the definition of $S_{c}$ and $S_{d}$ above are clearly not satisfied. Since our goal is to obtain the array contact times required to at least meet the $5 \%$ false negative criteria, we have taken a more conservative value for the determination of the MDL, using a value for $S_{d}$ that is equal to $4 \sigma_{0}$ for this higher background measurement, or 21,620 units for net signal within the $108 \mathrm{~cm}^{2}$ area.

Bare sheets were exposed to calibrated, planar sources having a defined dimension and measured total activity. The actual distribution of activity on the sources was unknown, and, as shown, is not generally uniform. Therefore, measured results cannot be analyzed for variation of pixel sensitivity or uncertainty in response because information on the sources is not available in this fine detail. Only gross response is obtained. Under the assumption that some environmental dose was obtained in array areas not intentionally exposed to the calibration sources, a local background signal is subtracted from the array readings in order to derive net signal induced by the characteristic source radiation. Available large area 


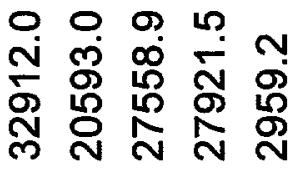

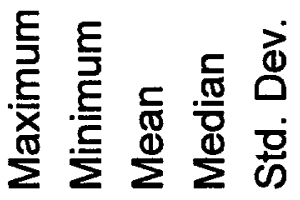

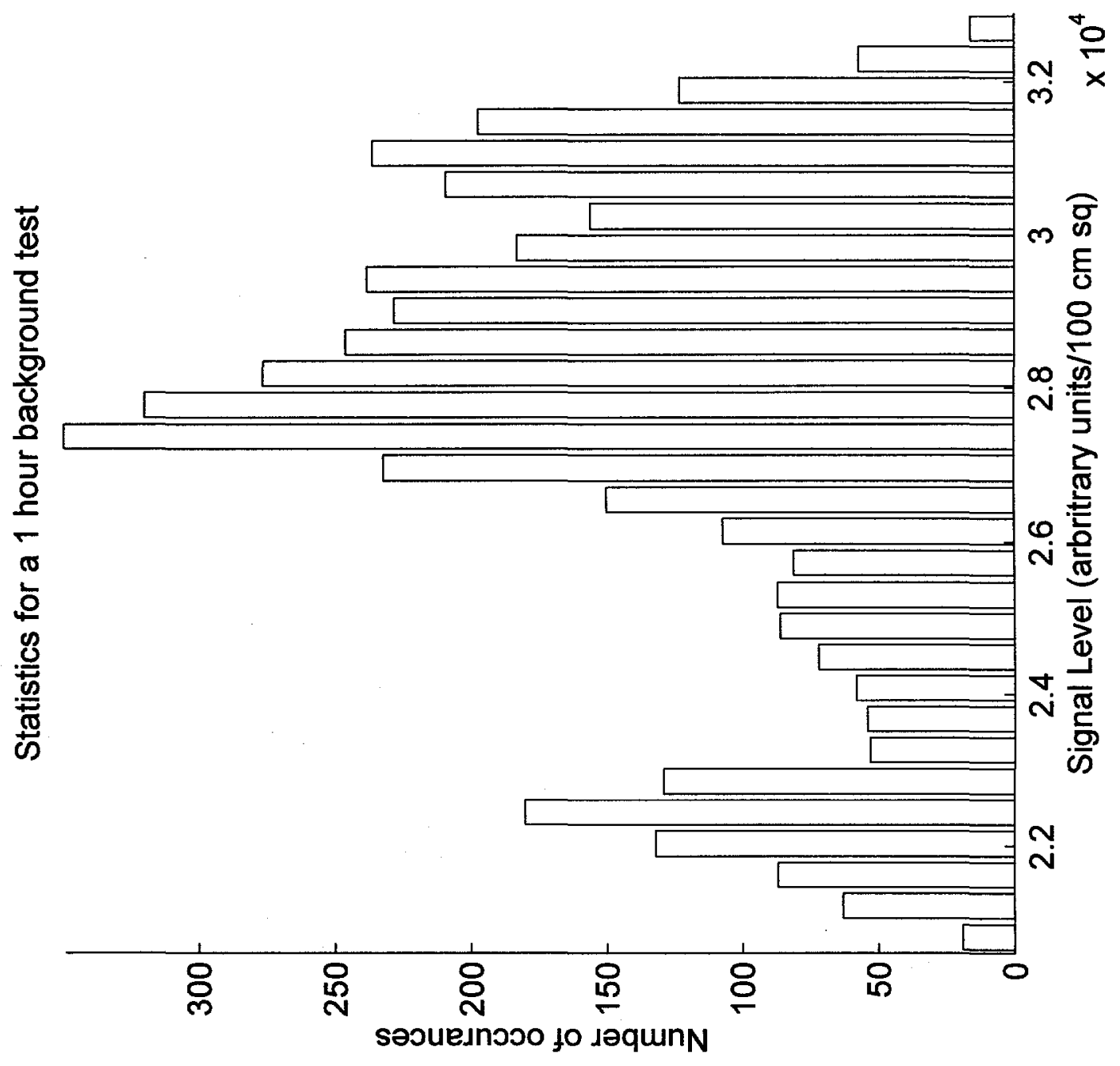




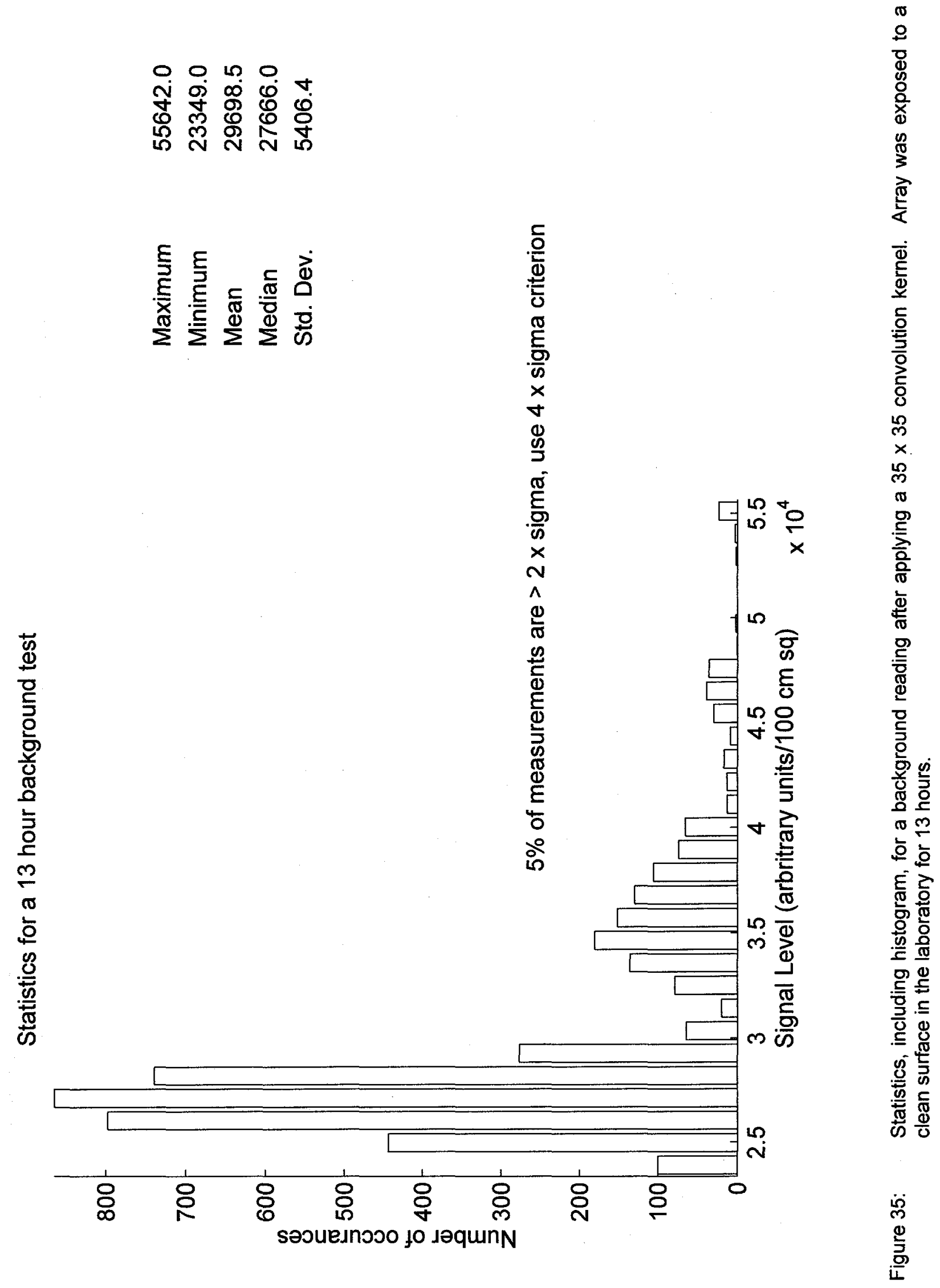


sources contained different activities of ${ }^{238} \mathrm{Pu}$ and ${ }^{241} \mathrm{Am}$ for alpha emitters (both Transuranics), ${ }^{90} \mathrm{Sr}$, ${ }^{99} \mathrm{Tc}$ for beta radiation, and ${ }^{137} \mathrm{Cs}$ for beta/gamma emission.

Figure 36 shows the result of exposing a sheet to a $7900 \mathrm{dpm}, 10 \mathrm{~cm} \times 15 \mathrm{~cm}\left(150 \mathrm{~cm}^{2}\right){ }^{241} \mathrm{Am}$ source for a period of 177 minutes. The total number of disintegrations per $100 \mathrm{~cm}^{2}$ occurring during this period is 932,200 averaged over the source. The average signal level obtained from this measurement is 3.93 units per dis $/ 100 \mathrm{~cm}^{2}$ within all $108 \mathrm{~cm}^{2}$ windows that could cover this source. Fig. 37 shows a second sheet which received two different exposures to this source, one of 6 minutes and one of 64 minutes duration. Fig. 38 shows the second result with the calibration factor of 3.93 units per dis $/ 100 \mathrm{~cm}^{2}$ applied.

The 6 minute exposure is intended to approximate a 60 minute exposure to a $500 \mathrm{dpm} / 100 \mathrm{~cm}^{2}$ source. Note that this source has an average activity of $5,267 \mathrm{dpm} / 100 \mathrm{~cm}^{2}$. The contour plots in Fig. 38 very nearly approximate the expected values. Note that systematic variation in sheet sensitivity has not been corrected. The maximum value obtained for the 64 minute exposure yields $4796 \mathrm{dpm} / 100 \mathrm{~cm}^{2}$, and the approximation to $500 \mathrm{dpm}$ source yields a maximum value of about $400 \mathrm{dpm} / 100 \mathrm{~cm}^{2}$.

Figure 39 shows a similar measurement made with a $660 \mathrm{~cm}^{2},{ }^{238} \mathrm{Pu}$ source having average activity distribution of $2000 \mathrm{dpm} / 100 \mathrm{~cm}^{2}$. The exposure period here is 150 minutes. Clearly the uniformity of the source is not good. With background subtracted (obtained from sheet area outside the source boundary), the net signal within a $108 \mathrm{~cm}^{2}$ window averages to be 4.02 units per dis $/ 100 \mathrm{~cm}^{2}$. Fig. 40 shows this same data with the calibration factor applied to the convolution output. The maximum value reported of $2386 \mathrm{dpm} / 100 \mathrm{~cm}^{2}$ agrees well with expectation. The minimum values shown in this plot result from convolution windows extending into unexposed area. Fig. 41 shows raw contour data following a 150 minute exposure of an array to a $660 \mathrm{~cm}^{2},{ }^{238} \mathrm{Pu}$ source having average activity of $200 \mathrm{dpm} / 100 \mathrm{~cm}^{2}$. Although there is no apparent source outline shown here, Fig. 42 shows the raw data convolved with the $35 \times 35$ kernel. The maximum returned activity is $148 \mathrm{dpm} / 100 \mathrm{~cm}^{2}$. Fig. 43 shows the statistical analysis of the convolved data shown in Fig. 42. Compare this result with the background reading shown in Fig. 35. If we assume that this strict $4 \sigma_{0}$ criteria for the MDL is exposure-time invariant (i.e., if we require that the 13 hour background count value be used for defining the MDL for much shorter exposure times), the equivalent activity derived from this criteria over the 150 minute exposure interval is equal to $36 \mathrm{dpm} / 100 \mathrm{~cm}^{2}$. The maximum value obtained from this plutonium exposure is about 4 times this equivalent detection limit.

Figure 44 shows the result obtained from a calibration exposure of a sheet to three $100 \mathrm{~cm}^{2}$ beta or beta/gamma sources. This exposure was made in order to obtain beta or beta/gamma response and derive minimum exposure times necessary to meet the release criteria. In this figure, the upper left exposure is to a $44,700 \mathrm{dpm}{ }^{90} \mathrm{Sr}$ beta source. This activity includes that of the equilibrium daughter ${ }^{90} \mathrm{Y}$. The upper right exposure is to a $227,000 \mathrm{dpm}{ }^{137} \mathrm{Cs}$ source and the lower center exposure is to a $43,000 \mathrm{dpm}$ ${ }^{99} \mathrm{Tc}$ beta source. All three exposures are of 208 minutes duration. Clearly, the ${ }^{90} \mathrm{Sr}$ source is highly nonuniform. The cesium source appears to be quite uniform. The data from this sheet was convoluted using a $35 \times 35 \mathrm{kernel}$ and the maximum value from each exposure region was obtained. Since all these sources are square, this maximum value represents the single best measure of aggregate response in a $100 \mathrm{~cm}^{2}$ area. The convolution result is shown in Fig. 45. No plot is available to demonstrate measurement of like sources in terms of $\mathrm{dpm} / 100 \mathrm{~cm}^{2}$.

The calibration results and MDL are summarized in Table 2. The MDL is obtained by finding the total number of disintegrations of each measured isotope within a $100 \mathrm{~cm}^{2}$ area required to meet the $4 \sigma_{0}$ detection limit criterion of the data shown in Fig. $35\left(4 \sigma_{0}=21,620\right.$ units in $108 \mathrm{~cm}^{2}$ window). 


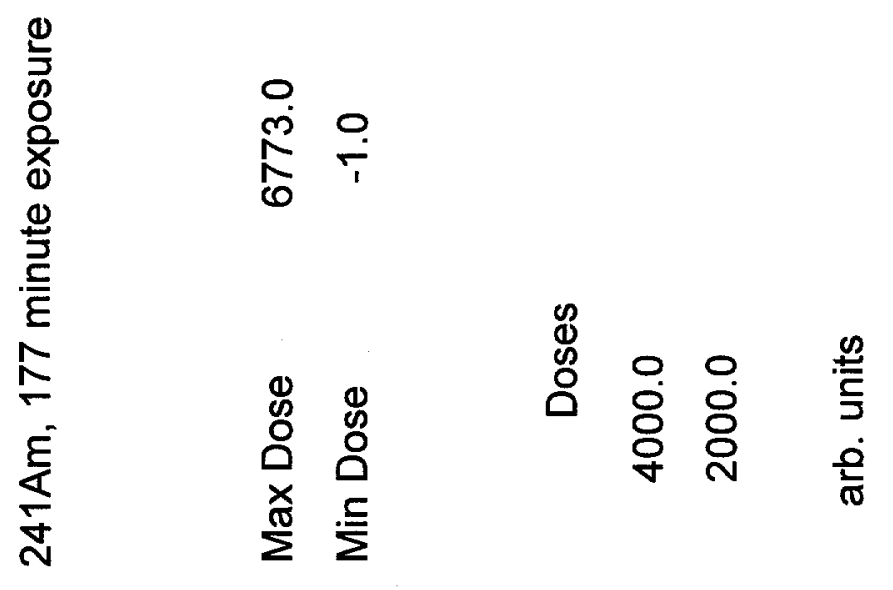

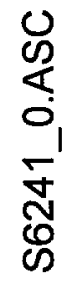

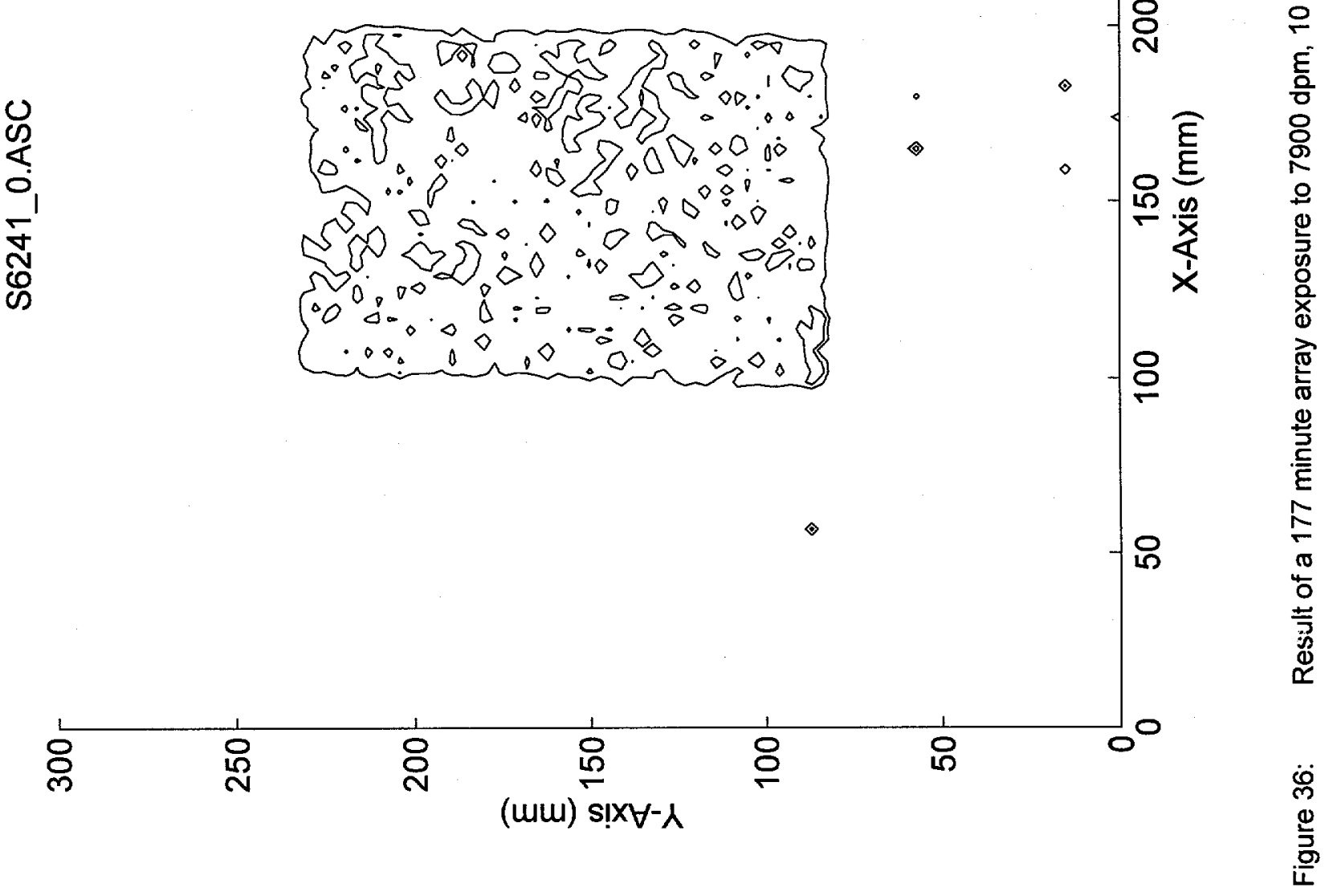




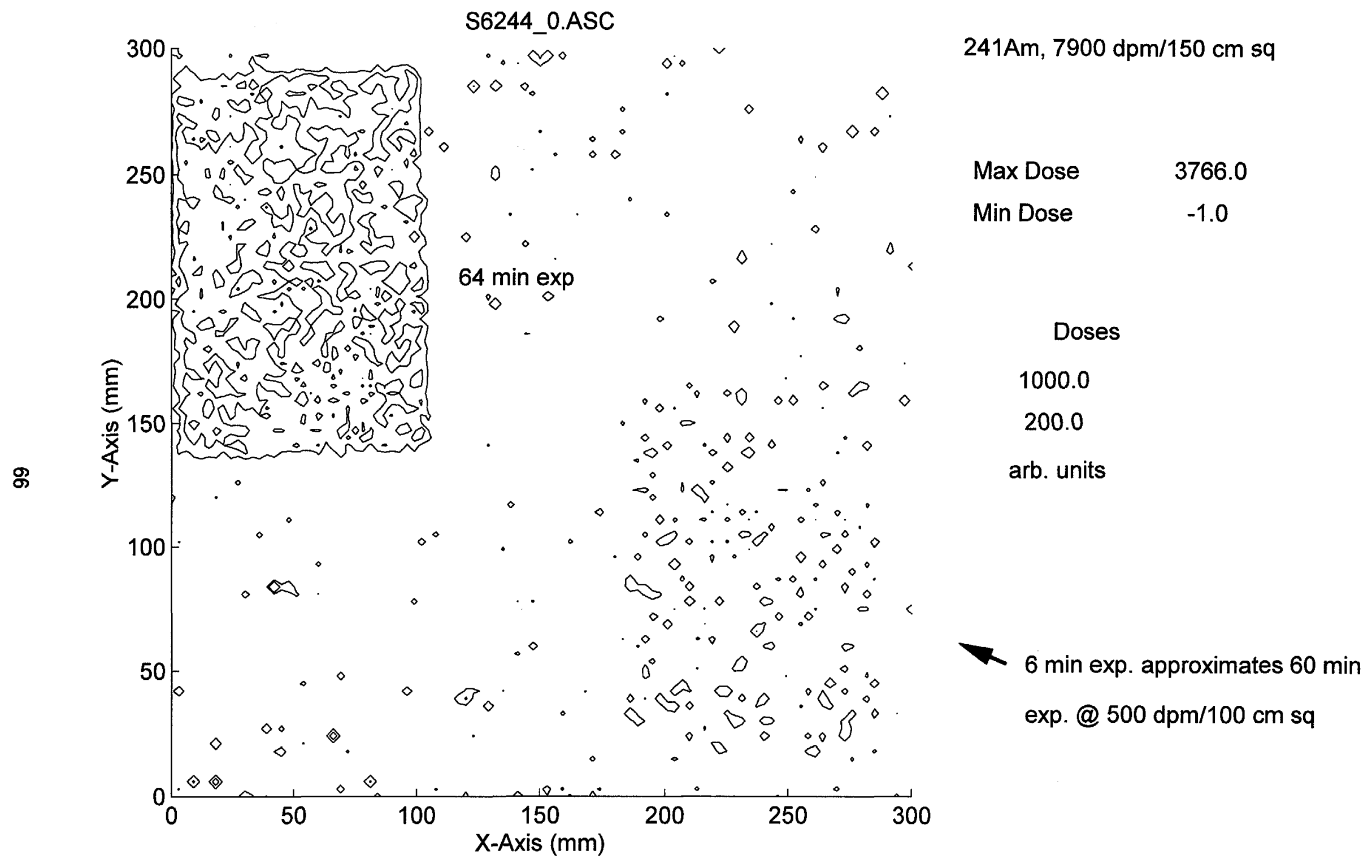

Figure 37: Result of 64 minute and 6 minute array exposures to $7900 \mathrm{dpm}, 10 \mathrm{~cm} \times 15 \mathrm{~cm}\left(150 \mathrm{~cm}^{2}\right)^{241} \mathrm{Am}$ source. 


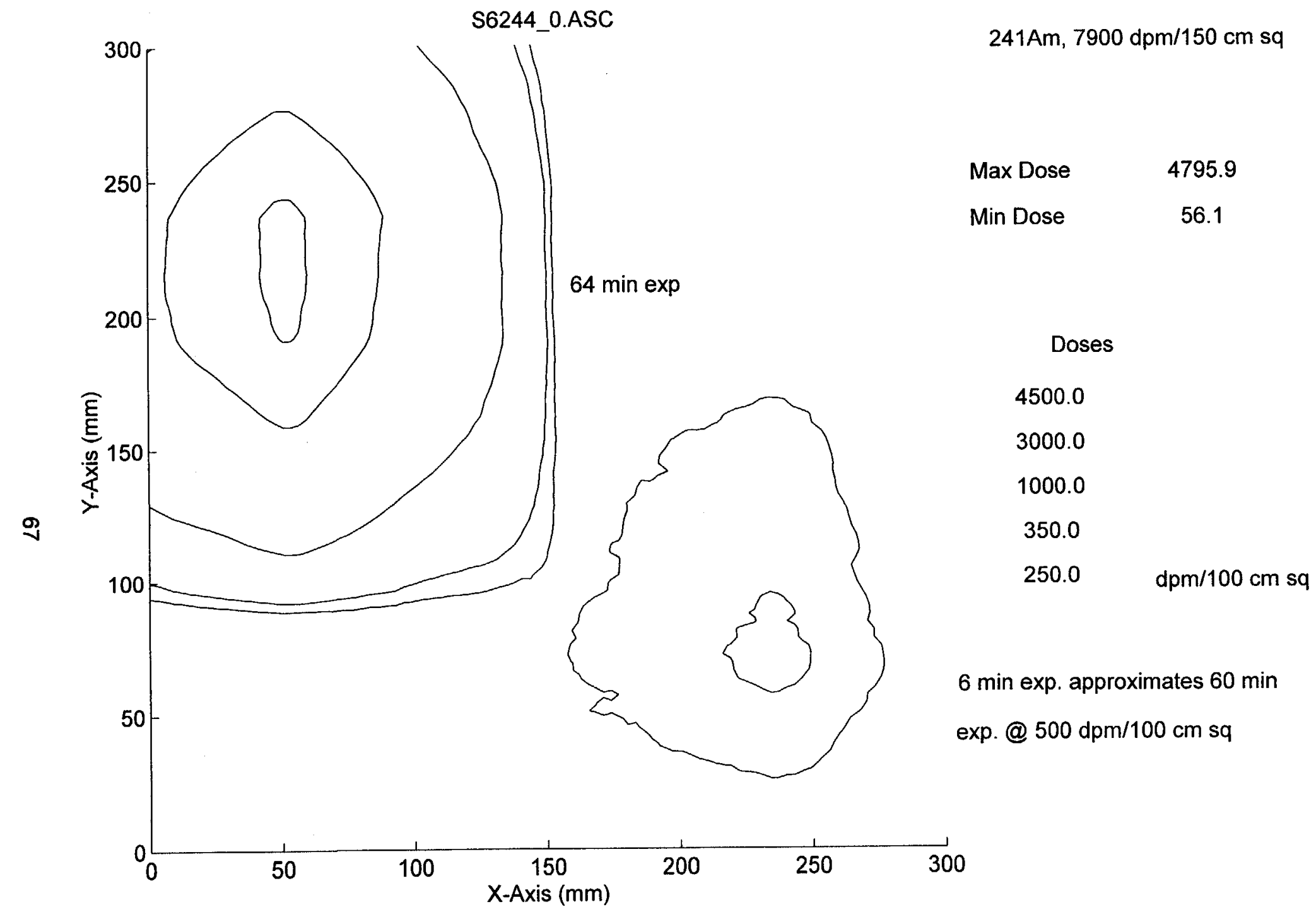

Figure 38: Result of applying the calibration factor of 3.93 units per dis/100 $\mathrm{cm}^{2}$ to Figure 37 . The six minute exposure is intended to approximate a 60 minute exposure to a $500 \mathrm{dpm} / 100 \mathrm{~cm}^{2}$ source. 


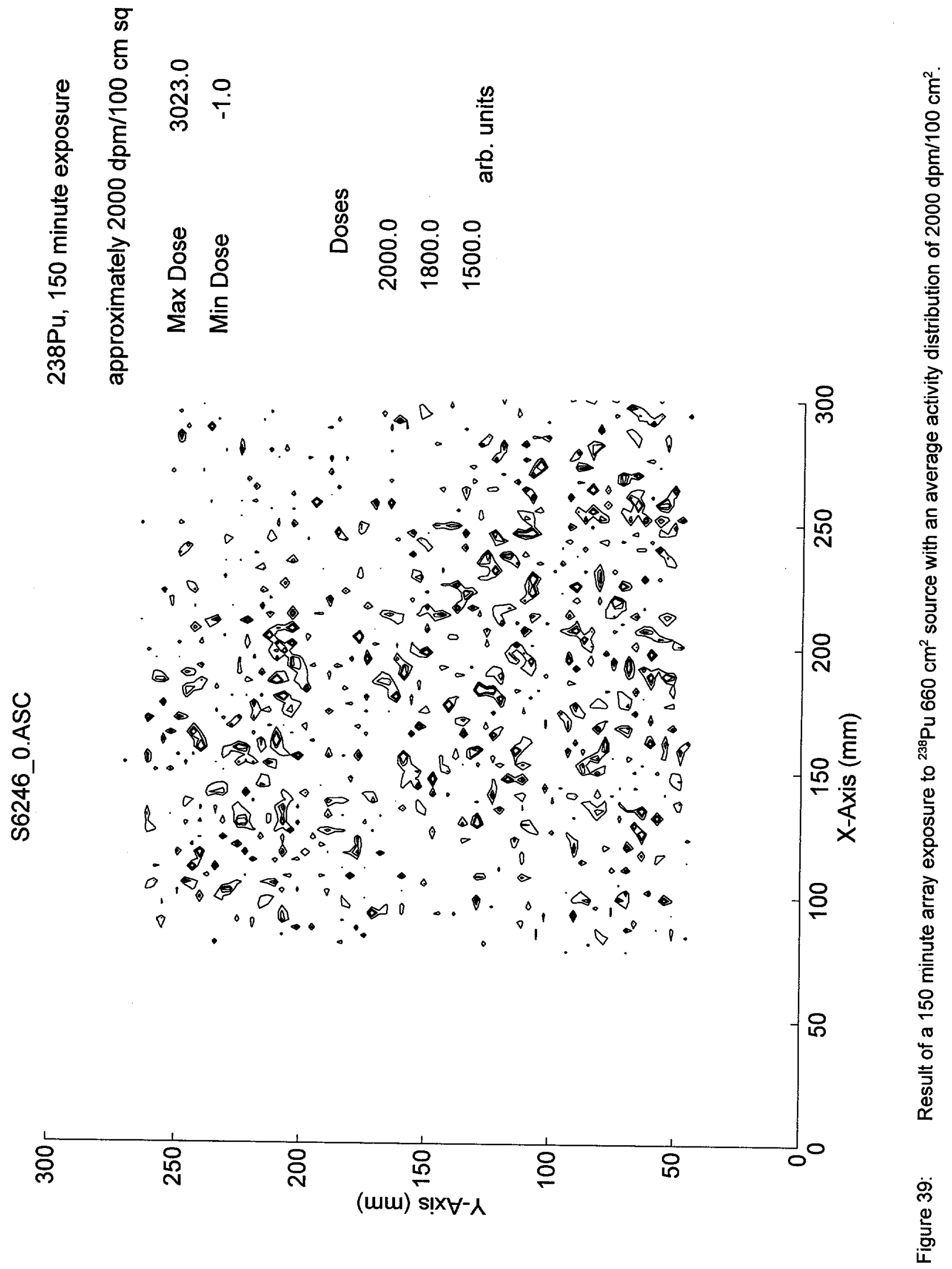




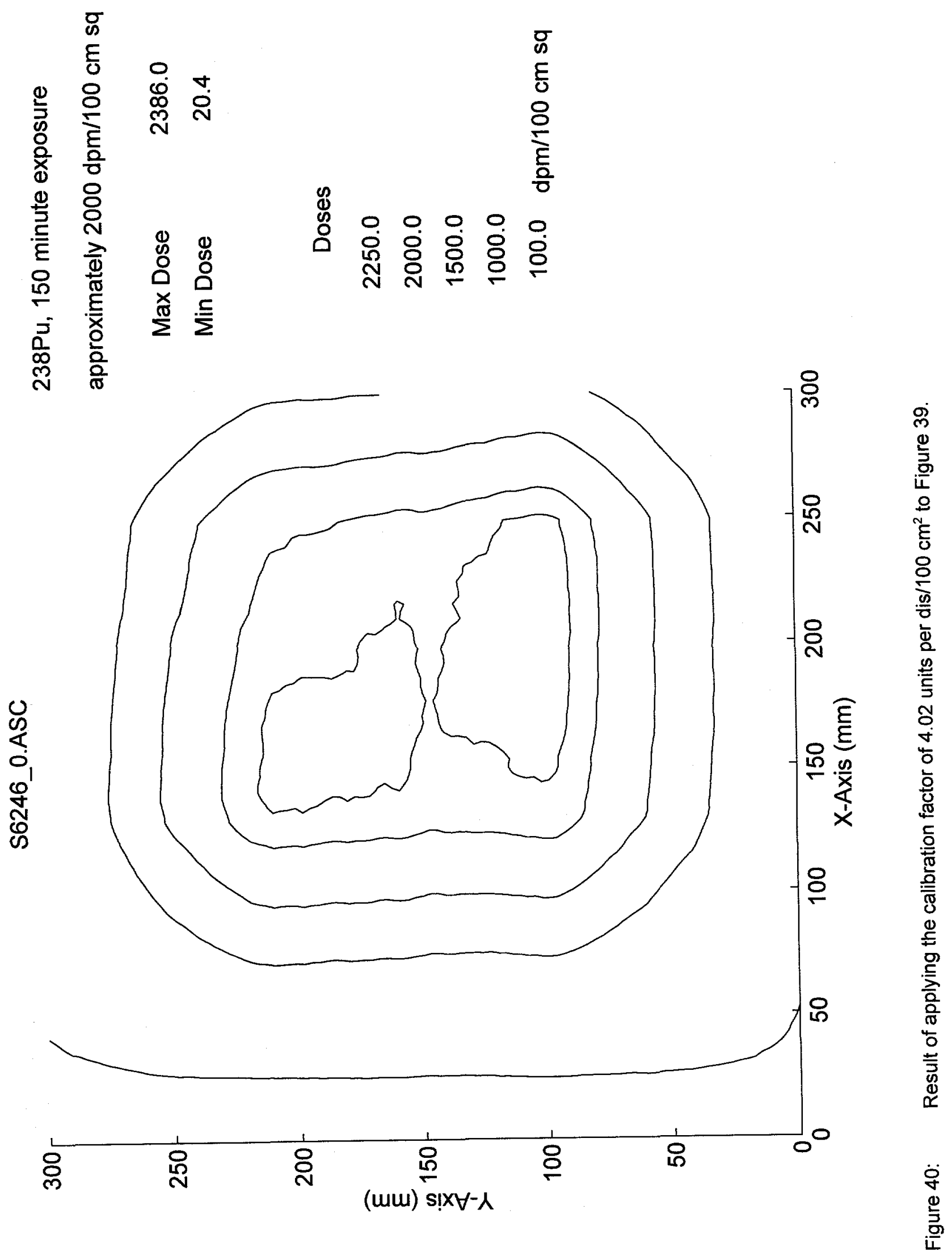




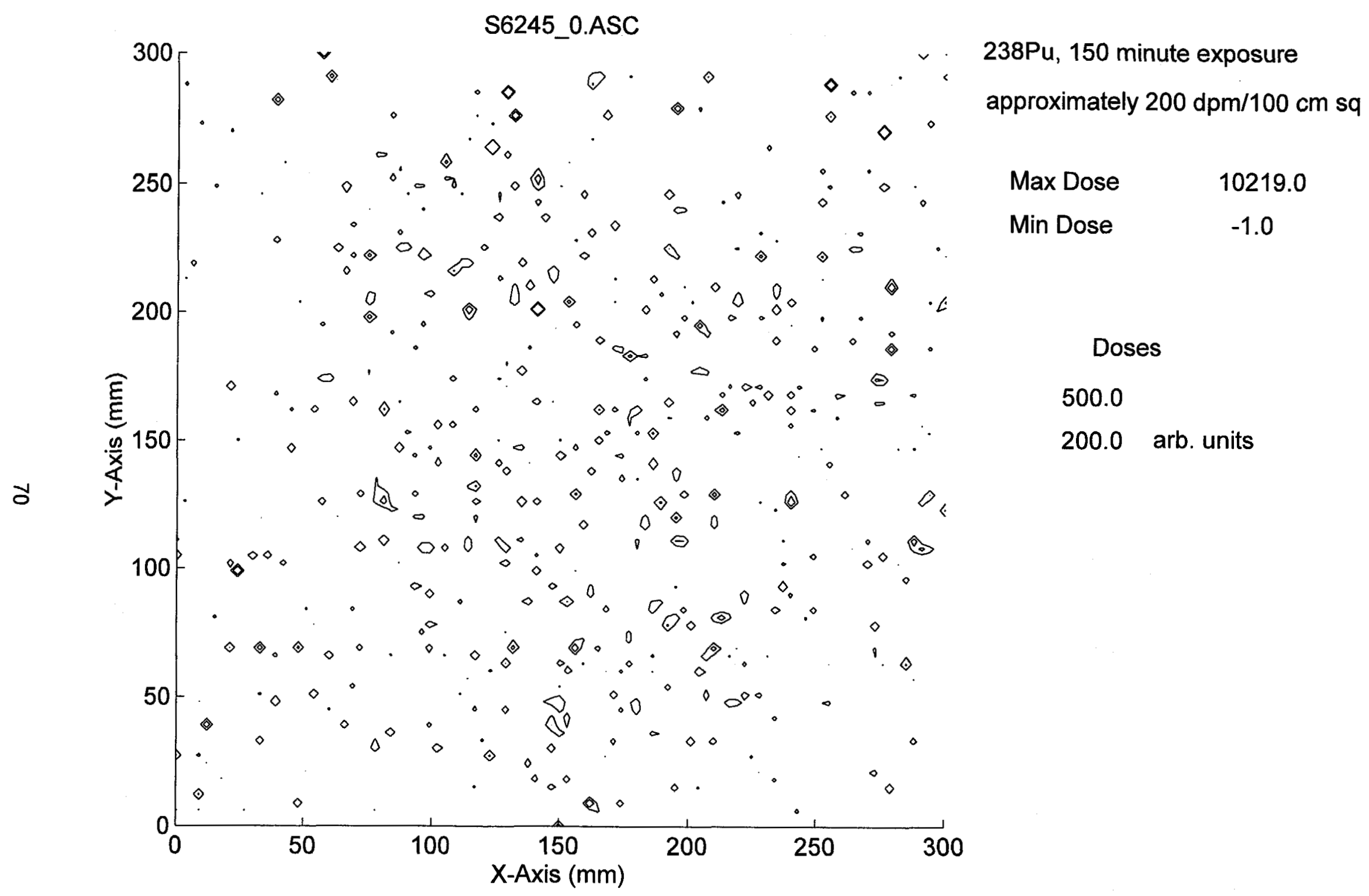

Figure 41: Result of a 150 minute array exposure to ${ }^{238} \mathrm{Pu} 660 \mathrm{~cm}^{2}$ source with an average activity distribution of $200 \mathrm{dpm} / 100 \mathrm{~cm}$. This source has one-tenth the activity of that used in Figure 39. Although there is no apparent source outline shown here, see Fig. 42. 


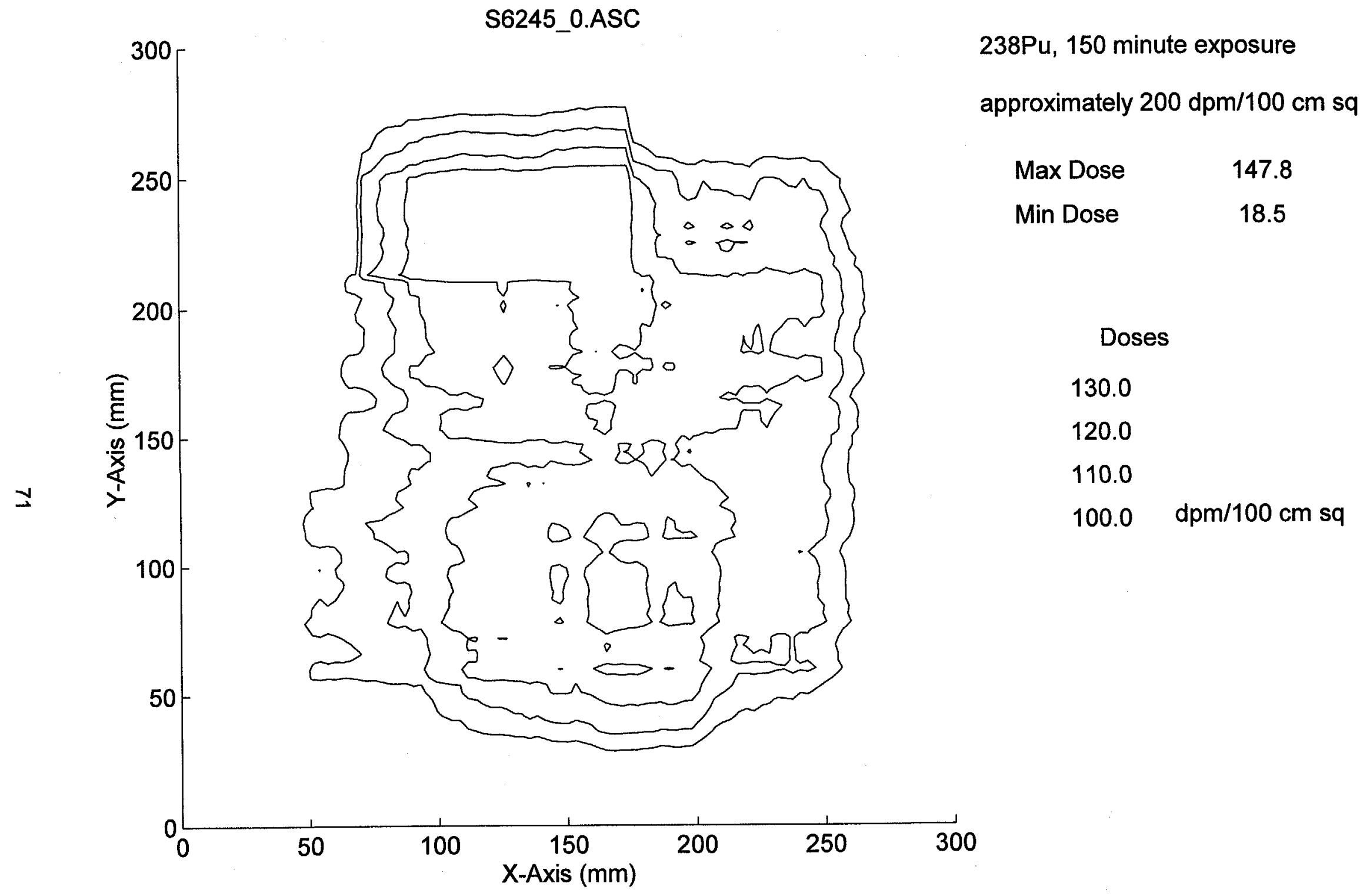

Figure 42: $\quad$ Result of applying a $35 \times 35$ convolution kernel and calibration factor to Figure 41 . 


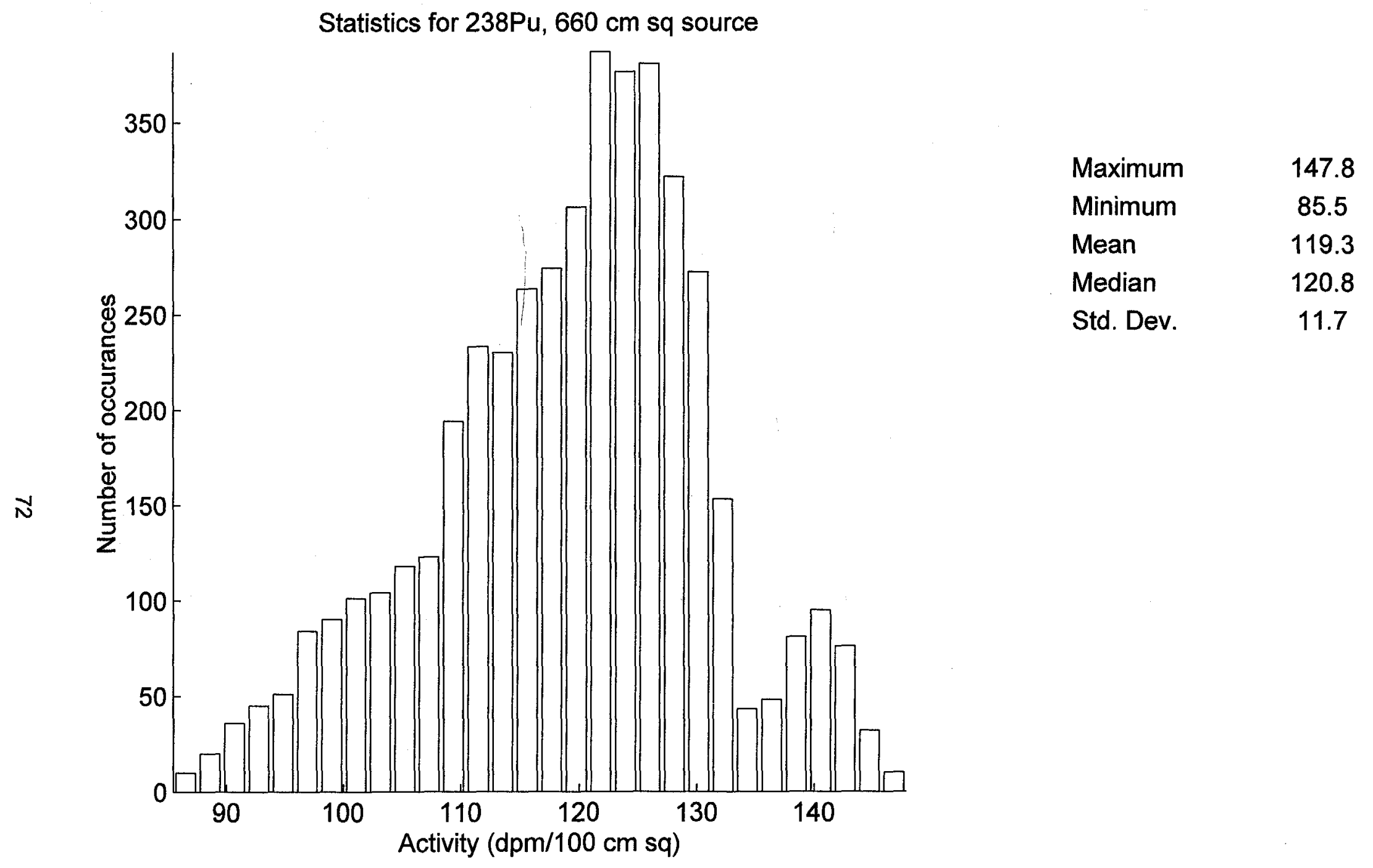

Figure 43: Statistics, including histogram, for Figure 42. Compare to the background reading statistics in Figure 35. 


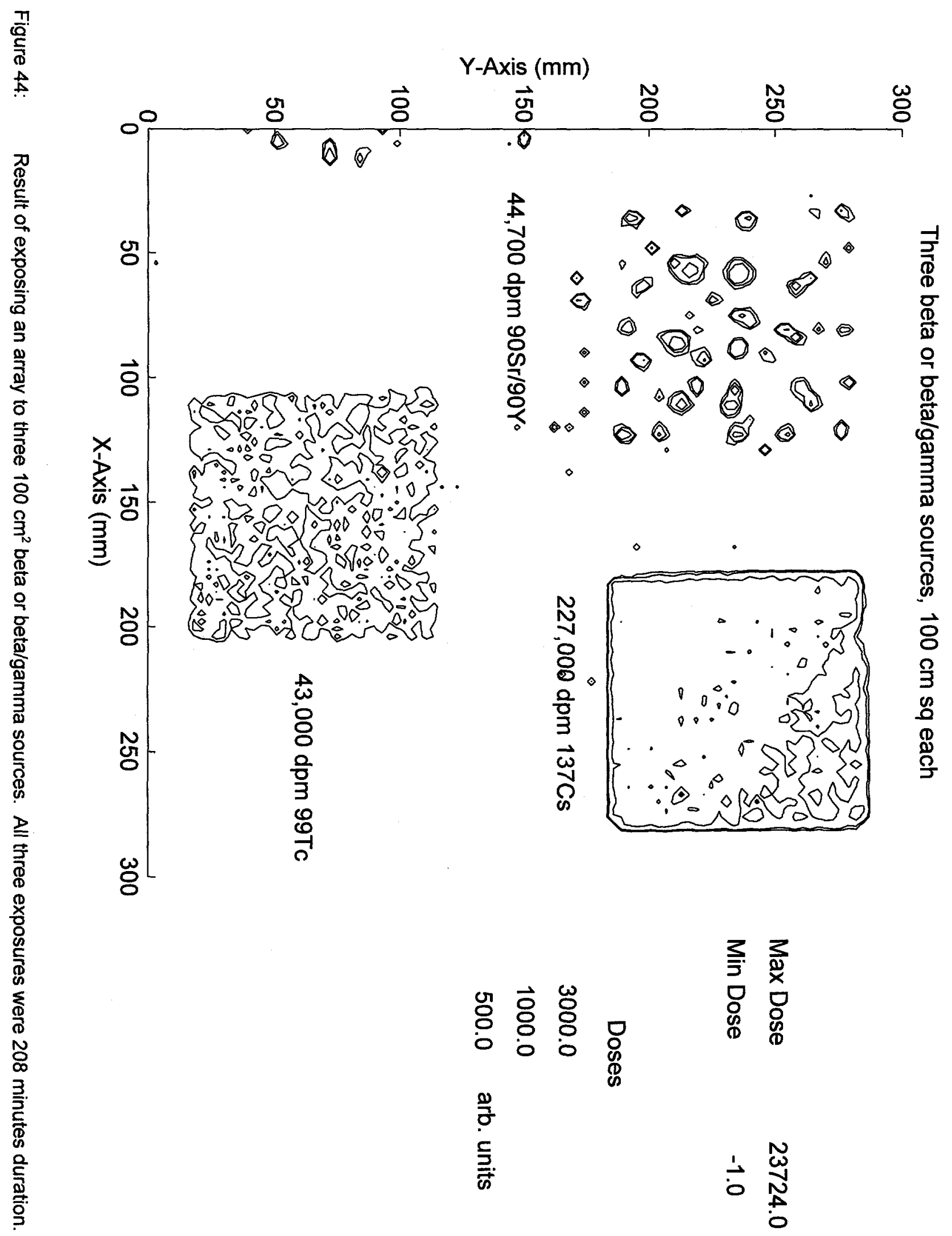



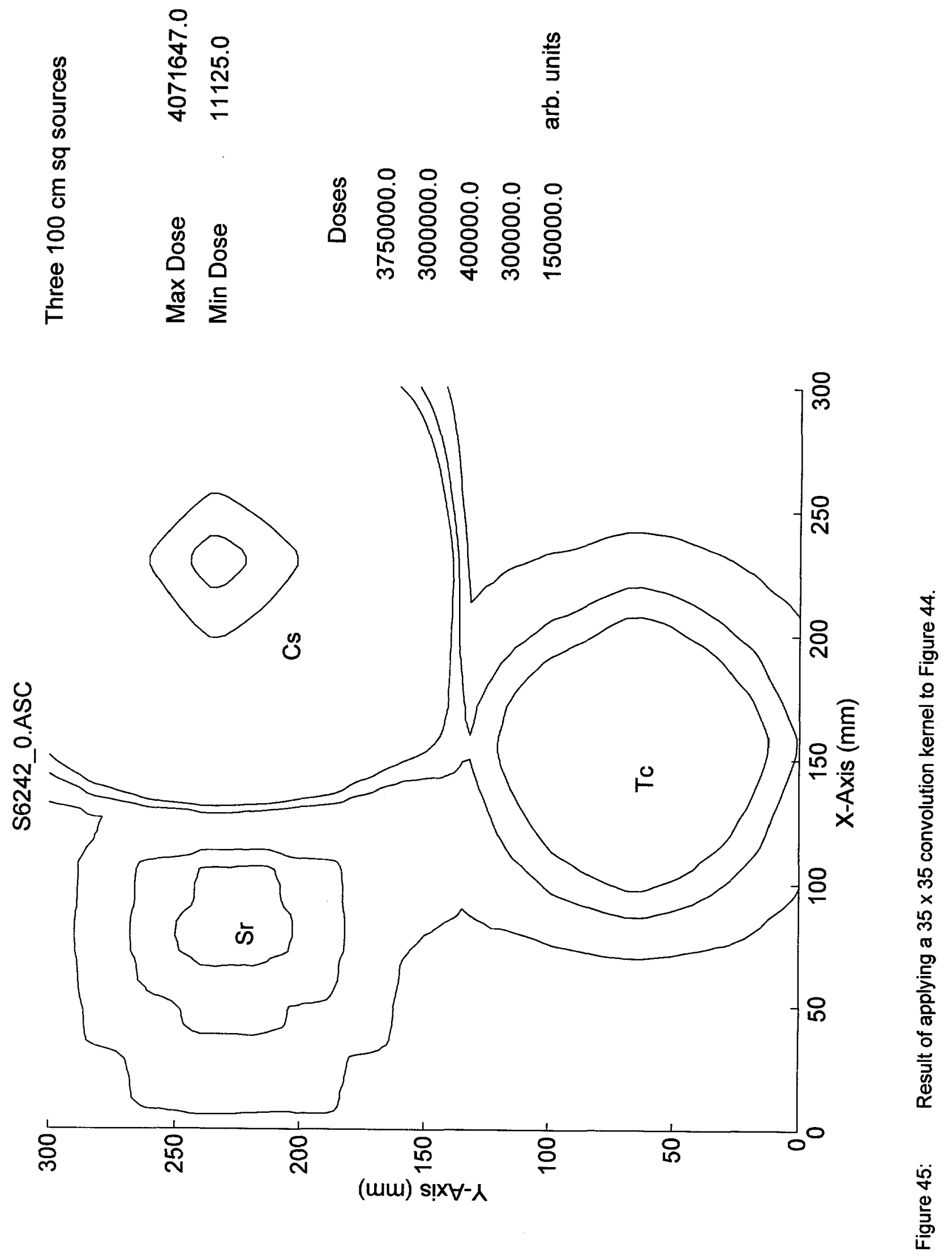
Table 2

Calibration Factors and MDL for Bare Arrays

\begin{tabular}{lcc}
\hline Isotope & $\begin{array}{c}\text { Calibration Factor } \\
\left.\text { (net signal, units per dis } / 100 \mathrm{~cm}^{2}\right)\end{array}$ & MDL (dis/100 $\left.\mathrm{cm}^{2}\right)$ \\
\hline${ }^{241} \mathrm{Am}(5.4 \mathrm{MeV}$ alpha) & 3.93 & 5,500 \\
${ }^{238} \mathrm{Pu}(5.5 \mathrm{MeV}$ alpha) & 4.02 & 5,380 \\
${ }^{238} \mathrm{U}(4.2 \mathrm{MeV}$ alpha) & 3.78 & 5,720 \\
${ }^{90} \mathrm{Sr}$ (beta) & 0.126 & 171,600 \\
${ }^{99} \mathrm{Tc}$ (beta) & 0.09 & 240,000 \\
${ }^{137} \mathrm{Cs}$ (beta/gamma) & 0.086 & 251,400 \\
\hline
\end{tabular}

No estimate of the uncertainty for these values can be derived because of the nature of the experiment. Only single exposures to large sources were obtained, and the unknown, but nonuniform, activity distribution in those sources preclude any meaningful statistical analysis of response variation. Further, systematic variation in array pixel sensitivity has not been accounted for. The values in Table 2 for ${ }^{238} U$ were obtained from the many measurements made during developmental testing.

\subsubsection{Performance in Background Radiation}

One final set of measurements was made on this project. The purpose of these measurements was to determine the ability of the system to detect localized surface contamination in the presence of a homogeneous background radiation field (emitted from regions other than the surface under measurement). Figure 46 shows the distribution of pixel response to an approximately uniform beam of ${ }^{137} \mathrm{Cs}$ gamma radiation. The exposure was performed with the array surface perpendicular to the beam axis, with the source facing the back of the array substrate. The phosphor surface was placed against an acrylic sheet but without any additional equilibration buildup material between the gamma source and the array. This was to simulate an actual field measurement. The approximate exposure was $47 \mathrm{mR}$. The results show the overall variation in pixel response to a uniform field, with an approximately $20 \%$ standard deviation.

Figure 47 shows a combination of various 30 minute ${ }^{238} \mathrm{U}$ exposures with a $20 \mathrm{mR}{ }^{137} \mathrm{Cs}$ gamma exposure as described above. The alpha exposure was performed after the gamma dose was delivered. In Fig. 47 the ${ }^{238} \mathrm{U}$ exposures are in three regions simulating a 30 minute $466 \mathrm{dpm}$ exposure within 100 $\mathrm{cm}^{2}$ windows having various distributions. Although these alpha exposures are visible above the background gamma dose, they are essentially undetected when a $100 \mathrm{~cm}^{2}$ convolution is applied to the raw data, as shown in Fig. 48. This is because the additional signal due to the alpha radiation is statistically insignificant in the variation of the sums obtained from the convolution algorithm. However, Fig. 49 demonstrates that a smaller convolution kernel, in this case $5 \times 5$, allows increased visibility of these sources.

The $20 \mathrm{mR}$ gamma exposure is intended to simulate the possibly allowable dose obtained by a person working in a fairly high background area. If this exposure were obtained every weekday, the approximate annual dose would be $5,000 \mathrm{mrem}$, the maximum annual occupational dose. This assumes 47 five-day weeks worked per year. As derived below, this level of background dose absorbed by the sheets is more than would be required in order to meet the measurement criteria of the release guideline limits in Table 1. 


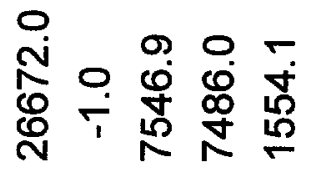

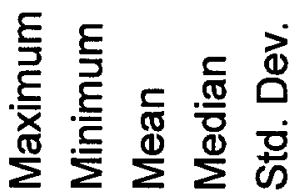

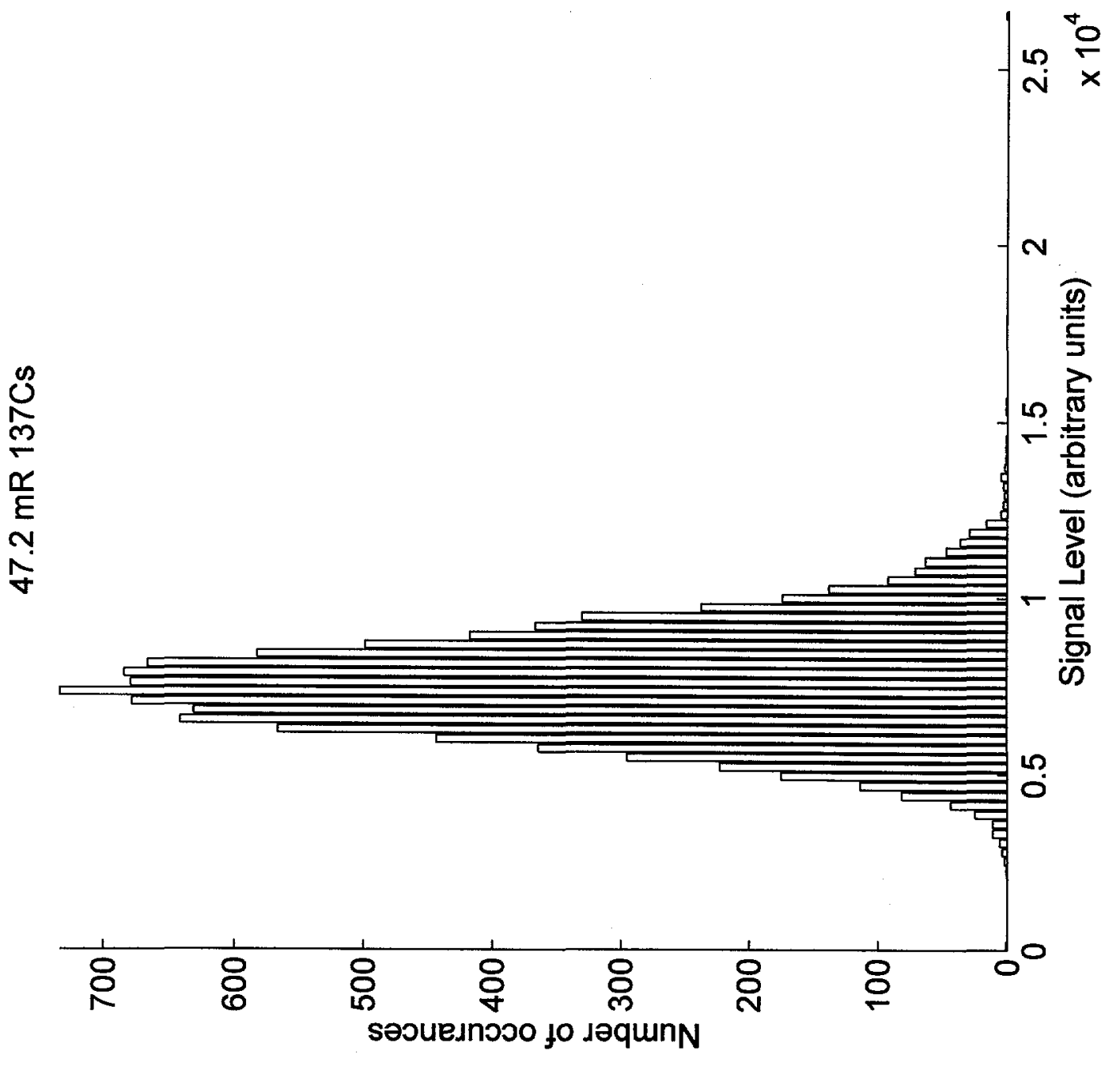




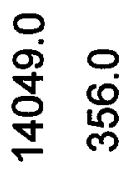

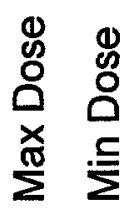

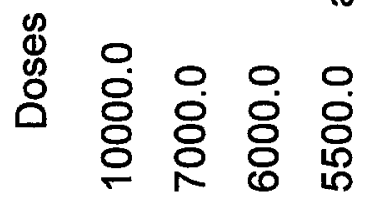

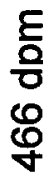

ᄅ্ৰ

$\stackrel{\frac{n}{5}}{5}$

은

范

은

疍

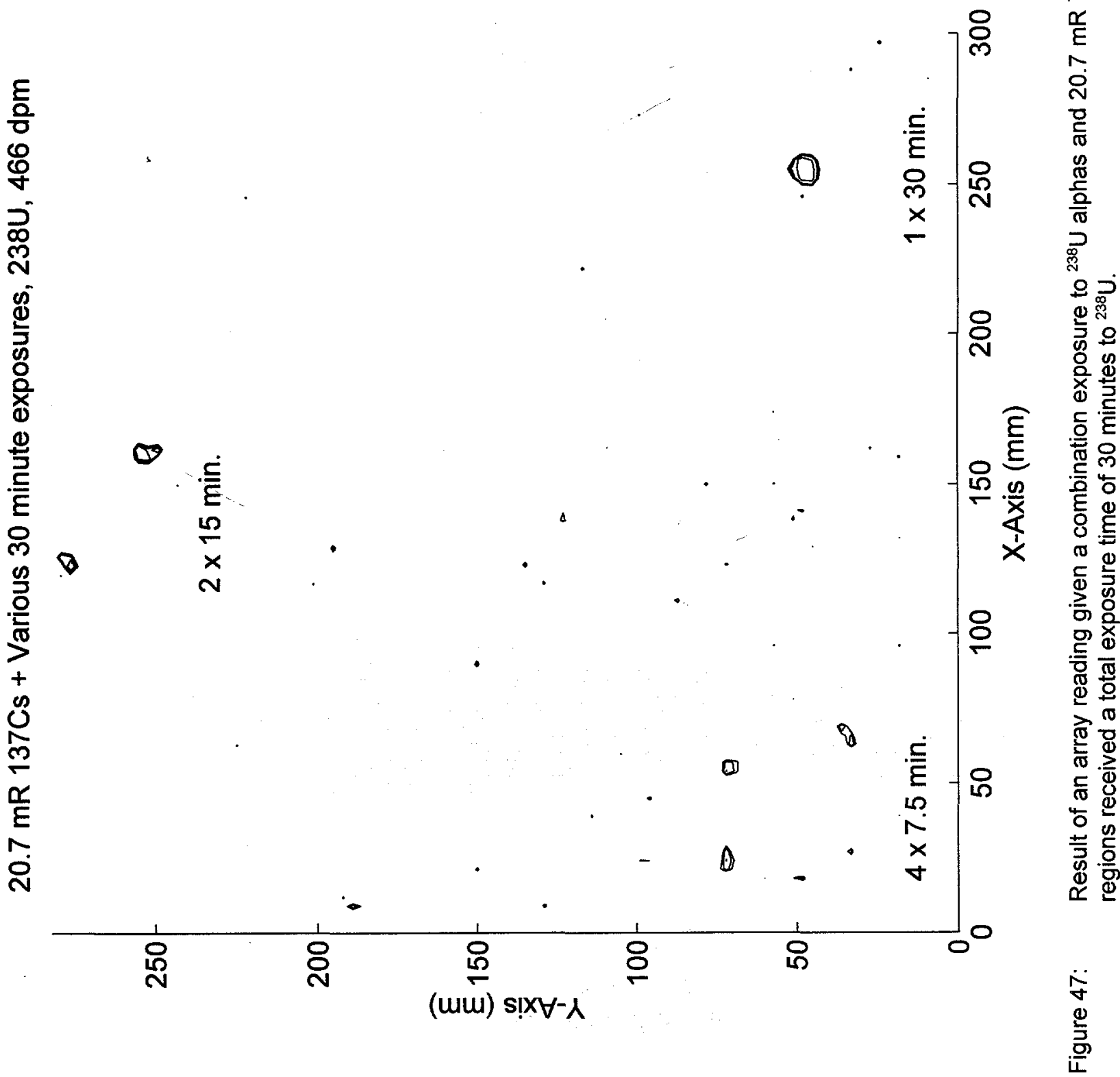




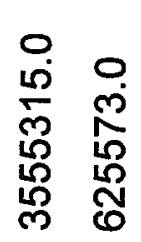

$\frac{0}{5}$
$\frac{.0}{5}$
$\frac{0}{6}$

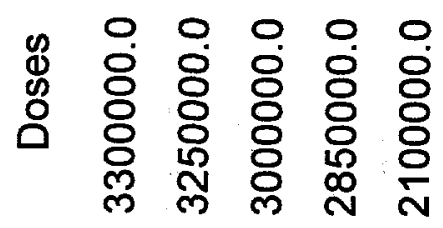

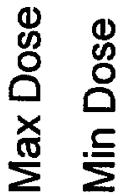

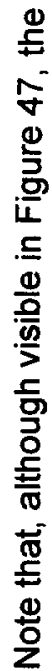

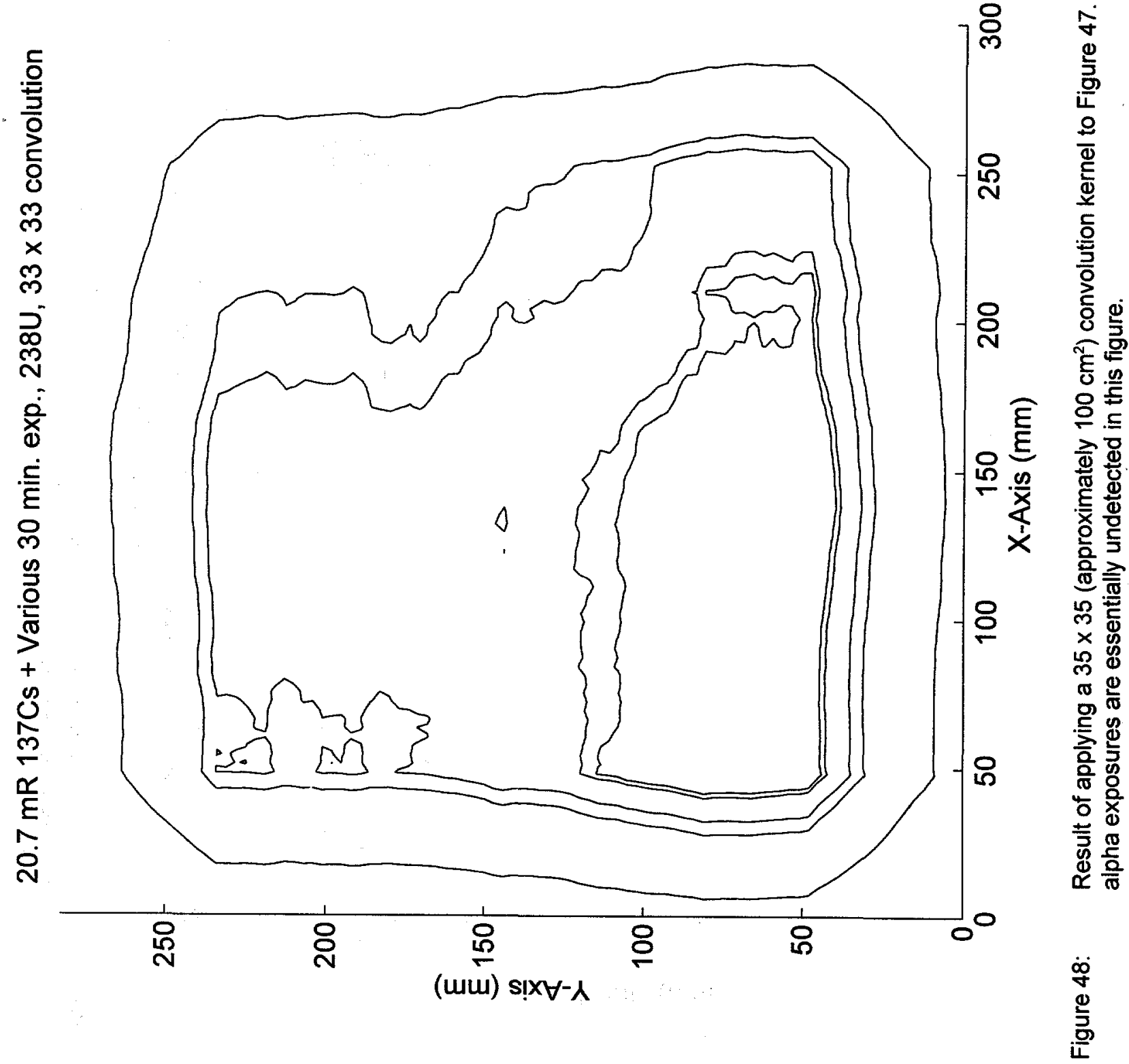




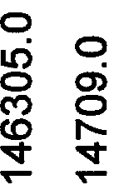

$\stackrel{0}{ \pm}$
$\frac{0}{5}$
$\frac{0}{\sigma}$

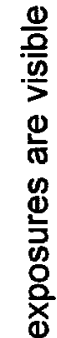

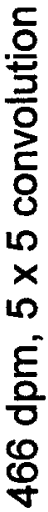

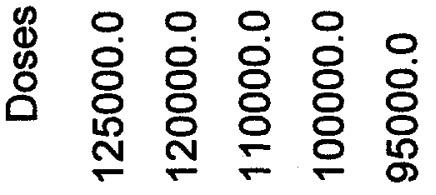

은

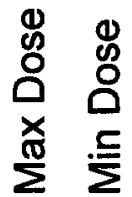

$\stackrel{\Perp}{=}$

莡

홈

틍

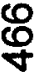

হ్ల

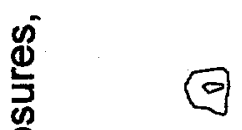

$\frac{0}{x}$

站 0

요

은

$+$

录

m

0.0

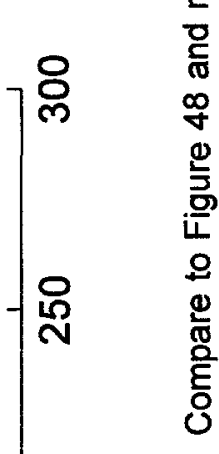

$\beta$

$\frac{\mathscr{\alpha}}{E}$

웅
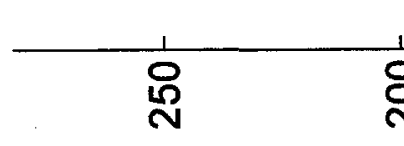

윰

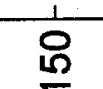

용

(um) s!x $\forall-\lambda$

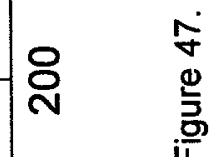

토

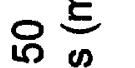

旁

这 혼

$\Delta$

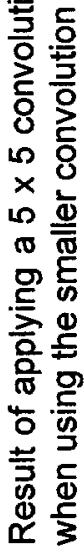

$\frac{9}{8}$
ํํㄴ 
Incorporating an array pixel sensitivity normalization algorithm was deferred in order to solve the fundamental problems discussed above. This is a simple routine to develop, in principle, and will be incorporated as development continues. If pixels were normalized, a background subtraction of uniform background radiation could easily be performed. This would allow reduction of the total signal to a level at which the incremental signal induced by local surface contamination would become statistically significant. Figure 50 is a simulation of what could be accomplished with a background subtraction and additional signal processing. Here we have subtracted a constant value slightly less than the peak values obtained from the four 7.5 minute alpha exposures, and then set to zero any of the differences resulting in a negative value. This latter operation is akin to thresholding (Russ, 1995). The resultant data array is then convoluted with a $33 \times 33$ kernel and displayed in Fig. 50. Compare this result with the convoluted raw data shown in Fig. 48. Again, this is a simulation to show what performance might be obtainable with normalization. In principle, a thorough characterization of background radiation is an integral part of the site survey process, and this information can be applied to surface contamination measurements. In addition, for sites or material being surveyed for unconditional release, the above assumed background dose rate is excessive and this measurement represents a probable near worst case of surface contamination measurement in a general background radiation field.

\subsubsection{Derivation of Minimum Contact Time for Measurement of Tested Isotopes}

Table 3 contains the derived minimum time required for bare arrays to be in contact with a contaminated surface in order to detect the guideline contamination limits with a $5 \%$ probability for false negative reading. The times are obtained by using the conservative estimate for MDL from Table 2 (MDL equal to four times the standard deviation of the instrument background signal for a 13 hour exposure to a clean surface). The contact time in minutes is obtained from the following formula:

$$
t(\min )=M D L / A_{L}
$$

Here, MDL is the minimum detectable number of disintegrations per $100 \mathrm{~cm}^{2}$ listed in Table 2, and $A_{L}$ is the guideline release limit, in $\mathrm{dpm} / 100 \mathrm{~cm}^{2}$. Given that the derived values in Table 3 for alpha emitters are on the order of one hour at $100 \mathrm{dpm} / \mathrm{cm}^{2}$, the standard deviation of the one hour background measurement may be more appropriate (see Fig. 34), in which case the contact times would decrease by nearly half. This possibility also applies to the beta and beta/gamma cases where the indicated time is on the order of one hour. These results were derived from measurements performed on bare arrays. In general, addition of a thin protective Mylar layer would require an approximate doubling of the exposure times for ${ }^{238} \mathrm{U}$, and a fairly small increase for the higher energy alpha emitters. 


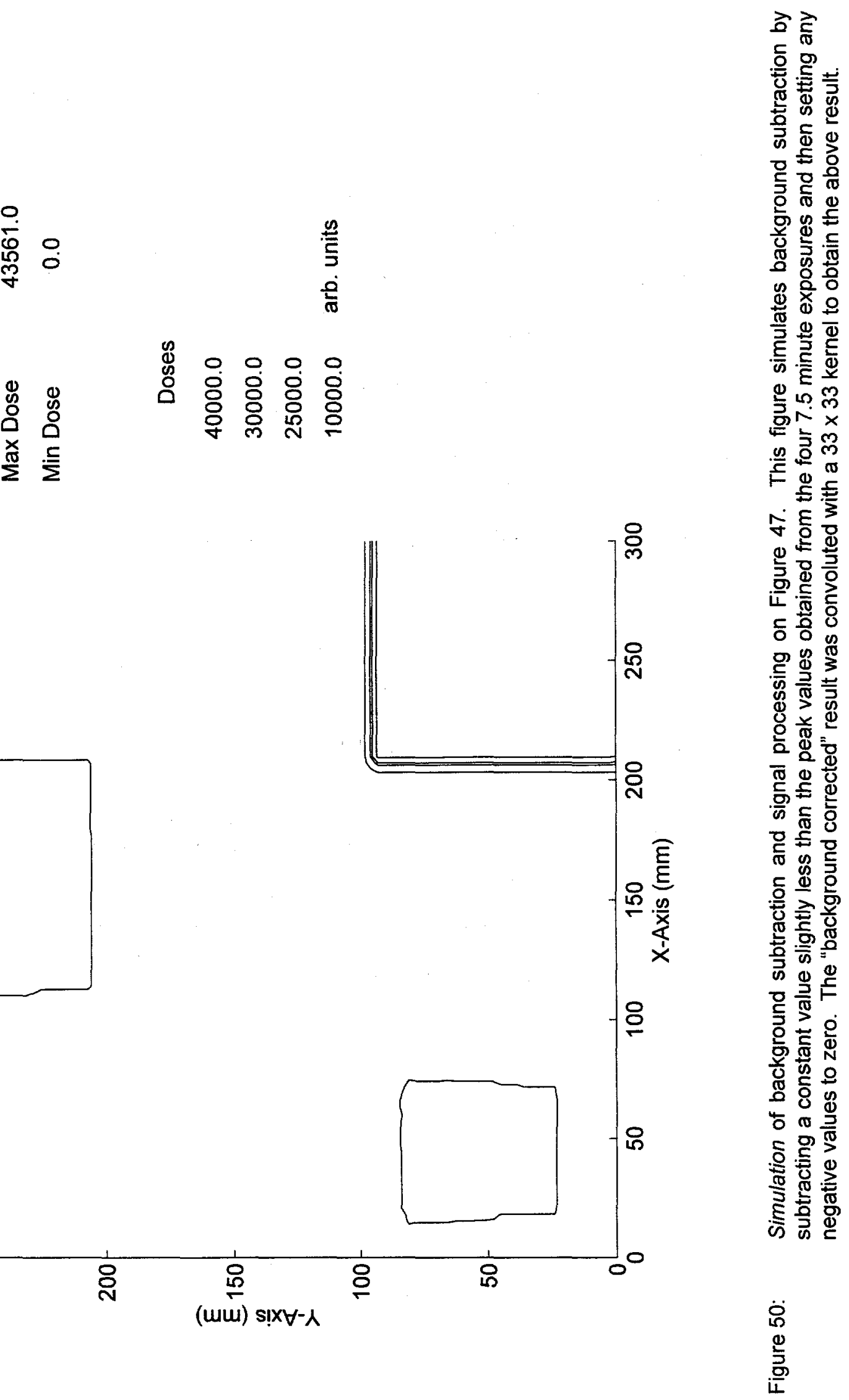


Table 3

Required Contact Time For Detection of Guideline Contamination Limits of Tested Sources

\begin{tabular}{cccc}
\hline NUCLIDE & $\begin{array}{c}\text { Limit For Average Sur- } \\
\text { face Contamination }\left(A_{L}\right)^{*}\end{array}$ & $\begin{array}{c}\text { Contact Time Required } \\
\text { for Detection of } A_{L} \\
(95 \% \text { Confidence Limit })\end{array}$ & $\begin{array}{c}\text { Contact Time Required } \\
\text { for Detection of } 3 \times A_{L} \\
(95 \% \text { Confidence Limit })\end{array}$ \\
\hline $\begin{array}{c}\text { Transuranics } \\
{ }^{241} \mathrm{Am},{ }^{238} \mathrm{Pu} \text { (alpha) }\end{array}$ & $100 \mathrm{dpm} / 100 \mathrm{~cm}^{2}$ & $55 \mathrm{~min}$ & $18 \mathrm{~min}$ \\
${ }^{238} \mathrm{U}$ (alpha) & $5,000 \mathrm{dpm} / 100 \mathrm{~cm}^{2}$ & $1.2 \mathrm{~min}$ & $0.4 \mathrm{~min}$ \\
${ }^{90} \mathrm{Sr}\left(+{ }^{90} \mathrm{Y}\right)$ (beta) & $1,000 \mathrm{dpm} / 100 \mathrm{~cm}^{2}$ & $170 \mathrm{~min}$ & $57 \mathrm{~min}$ \\
$\quad \begin{array}{c}{ }^{99} \mathrm{Tc} \text { (beta) } \\
{ }^{137} \mathrm{Cs} \text { (beta/gamma) }\end{array}$ & $5,000 \mathrm{dpm} / 100 \mathrm{~cm}^{2}$ & $48 \mathrm{~min}$ & $16 \mathrm{~min}$ \\
\hline
\end{tabular}

* From Table 1

\subsection{Phase II Discussion and Conclusions}

\subsubsection{Summary}

The results obtained above demonstrate the level of system improvement achieved in Phase II, despite the difficulities encountered. An overall improvement of a factor of at least 20 in required exposure times to detect release limits has been achieved since the end of the Phase I effort. Of the eight tasks listed above for the Phase II effort, only the sixth and seventh were totally omitted. Some field testing was done, but not with the fully optimized system.

Given the above results, this project has yielded a potentially valuable new capability in the measurement of low-level surface contamination. It had been widely believed that it was virtually impossible to detect transuranic alpha emitters at levels as low as the values listed in Table $\mathrm{l}^{\mathrm{h}}$, thereby eliminating the possibility of releasing suspected material or facilities to the general public. Although rigorous field testing of the system remains to be performed, these results show promise that this system may be a viable candidate for performing general contamination surveys for sites to be released.

A method has been developed that allows for detection of these low levels of surface contamination with active detectors. This method requires contiguous, 20 second static measurements, in which a $100 \mathrm{~cm}^{2}$ surface alpha probe is held in contact with the surface for 20 seconds, and placed on a precisely adjacent (contiguous) area for the next 20 second interval, and so forth. If a single click is heard, that is significant, and a second interval is counted on the same surface segment. If a click is again heard, that

\footnotetext{
${ }^{\mathrm{h}}$ Personal Communication, M. Johnson (1996).

' lbid.
} 
segment is contaminated above the limit (with a maximum of $5 \%$ false negative readings, from statistics). The procedure is tedious and error prone. Furthermore, technicians work in pairs, one doing the measurement, one observing for compliance with the protocol. To survey an absolutely clean surface of $1 \mathrm{~m}^{2}$, it would require 66 minutes each of two technicians' time (assuming not a single click is heard).

The TLD system has obvious operational advantages in comparison with this method, and further, the above described method works only for accessible surfaces. The TLD sheets are in static contact with the surveyed surface, and human factors are not relevant during the exposure interval. Further, technicians can lay many square meters of TLD sheets in an hour on accessible surfaces. This, unfortunately, does not account for reading time. In principle, the surface contamination sensitivity of the TLD arrays is arbitrarily low, given sufficient contact time, and therefore the method has many attractive attributes. The drawbacks of the system are an absolute inability to discriminate between different alpha emitting nuclides (that of principle concern being natural radon, with other natural nuclides having much higher release limits), and potential deployment cost. Alpha/beta discrimination is fairly trivial; covering an array with an appropriate thickness of material will eliminate the alpha response. Foreknowledge of potential contaminants is generally part of a contamination survey of facilities, and therefore lack of isotope discrimination may not preclude adopting this system for some aspects of contamination survey methodology. If coarse scanning is accepted, rates of 15 minutes per sheet may even have a favorable economic comparison with contiguous static surveys for release of accessible "clean" surfaces.

Even if the Laser TLD system turns out not to be cost competitive with use of active instruments for measuring fairly large expanses of accessible surface area, the unique capabilities of this system require examination. The principle application here is the measurement of surfaces inaccessible to standard survey instrumentation, specifically the measurement of curved surfaces having radii too small to allow an alpha probe to remain within the maximum allowed distance from the surface. The obvious example is piping and duct work. The thin, flexible sheets have an obvious application to this problem, as the arrays can conform to cylindrical surfaces, without any required correction factors for area projections, etc.

Many of the potential applications of this system are discussed above in Section 2.3. The Phase II effort reinforces and makes more attractive the application of this system's capabilities to the measurement problems mentioned.

\subsubsection{Further Development}

Before full commercialization of this system can be achieved, a number of areas that were deferred during this project need to be addressed. Primarily, these are developing routines for array pixel normalization and subtraction of background dose from surface measurement data. An actual field test needs to be performed at a contaminated site. Additionally, further array fabrication improvements need to be developed in the areas of uniformity of pixel response (perhaps obviating the need for array normalization) and gradual loss in sensitivity due to binder darkening. The latter two factors impact the economic viability of the system.

The reader has the capability of processing sheets faster than the standard one-hour interval for full resolution reading. The development of the intelligent coarse/fine scan algorithm allows the reader to samples sheets at a rate of up to 4 per hour. The question is whether this sampling rate is adequate to meet measurement requirements. This question will need to be explored with pertinent experts.

Finally, the sheets have proved to be fairly robust, surviving the abrasion of soil insertion and general use. The arrays used to produce the final measurements and results had been used dozens of times each during the project. The use of the automated glow curve examination technique has proved 
quite successful, adding to the increased lifetime of the arrays. Aside from the gradual sensitivity loss, the arrays are very durable. The issue is whether packaging is actually required. Given the nature of the intended purpose (the possibility of array contamination or cross contamination of surveyed sites exists), it seems obvious that array packaging should be used. The packaging would also be desirable for electrostatic protection. Therefore, the issue is the disposal of the packaging waste, and needs to be examined. 


\section{SBIR PHASE III}

As part of the SBIR program, a contractor receiving Phase II funds for innovation and development work generally makes a commitment to see that private investment goes to further develop and commercialize the SBIR Phase I and II work. The original contractor, International Sensor Technology, made such a commitment and considers its sale to Keithley Instruments in December of 1995 as a positive indication that this commitment has been fulfilled. The assigned successor to this contract, Keithley, recognized the commercialization potential of Laser TLD in acquiring the rights to IST developments. As a continuation of the SBIR program under Phase III, Keithley is presently formulating a business plan for Laser TLD in general, and specifically researching the radioactive waste management market.

The development of integrating 2-dimensional Laser TLD detectors for low-level radioactive contamination on surfaces has already caught the attention of potential customers. The Waste Management Conference in March 1997 in Tucson, AZ, provided invaluable information for this effort. In intensive discussions with representatives from major players in industry and DOE, a number of applications have been identified, particularly in the areas of measurement of surfaces contaminated with transuranic alpha emitters and low-energy beta emitters.

Specifically, the following applications of sheets in characterizing residual contamination on surfaces were considered:

1. Detection and measurement of localized contamination at and above the NRC release levels in pipes and, generally, on surfaces that are not readily accessible to alpha detectors or that cannot be removed for measurement with ion detectors. The sheets can be configured in various shapes (large monolithic arrays of TLDs, narrow strips, long tapes, etc.)

2. Detection of low-level contamination by non-penetrating radioactivity inside a waste drum between the inside wall and the plastic liner.

3. Characterization of technetium contamination at and above the release level on stainless steel baffles in uranium diffusion plants.

Initially, a sheet reading service may be offered to DOE facilities and industry. This will provide additional experience needed for future deployment of Laser TLD sheets of various configurations on different surfaces. Valuable insight is to be gained on handling the sheets in the field and on data processing. This will further the determination of the economical viability of this new technology. The result will be establishment of detailed criteria for engineering and production of fieldable sheet readers and low cost sheets within the next two years. 



\section{REFERENCES}

Bloomsburg, C.D., et. al., "A New Desktop Laser-Heated TLD Reader," Radiat. Prot. Dosim. 34, 365 (1990).

Braunlich, P., et. al., "Laser Heating of Thermoluminescence Dielectric Layers," Appl. Phys. Lett. 39, 760 (1981).

Braunlich, P., "Present State and Future of Laser Heated TLD," Radiat. Prot. Dosim. 34, 345 (1990).

Currie, L.A., Lower Limit of Detection: Definition and Elaboration of a Proposed Position for Radiological Effluent and Environmental Measurements, NUREG/CR-4007, 1984.

Jones, S.C., et al, "Comparison of Laser and Conventional Heating in TLD Dosemapping," Rev. Sci. Instrum. 63, 4898 (1992).

Jones, S.C., "Laser TLD 2-Dimensional Dosemapping," Radiat. Prot. Dosim. 47, 457 (1993).

Martin, J.B., D.M. Fleming and K.L. Swinth, "Practical Limitations on Alpha Contamination Monitoring," abstract, Thirty-Fifth Annual Meeting of the Health Physics Society, paper THPM-B4.

Meyer, K.E., R.B. Gammage, C.S. Dudney, I.M. Angelini, "Method for Utilization of Electret Ionization Chambers for Characterization of Gross Alpha Emission from Indoor Surfaces," DOE Methods for Evaluating Environmental and Waste Management Samples, RA010, 1994.

Oldfield, B.W., Feasibility of Using Two-Dimensional TLD Arrays to Measure Residual Surface Contamination on Inaccessible Surfaces, Thesis, Master of Science, Washington State University, 1995.

Russ, J.C., The Image Processing Handbook, CRC Press, 1995.

Sommers, J.F., "Sensitivity of Portable, Beta-Gamma Survey Instruments," Nuclear Safety 16, 452 (1975).

US Atomic Energy Commission Regulatory Guide 1.86 Termination of Operating Licenses for Nuclear Reactors (1974). See also Radiation Control Manual, US Department of Energy (1992). 
2. TITLE AND SUBTITLE

Measurement of Residual Radioactive Surface Contamination by

2-D Laser Heated TLD

NUREG/CR-6037

5. AUTHORIS

S. C. Jones

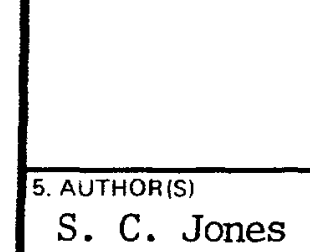

8. PERFORMING ORGANIZATION - NAME AND ADDRESS IIf NRC, provide Division, Office or Regjon, U.S. Nuclear Regularory Commission, and mailing address; if contractor, provide name and mailing address.)

Keithley Instruments, Inc.

28775 Aurora Road

Cleveland, OH 44139

9. SPONSORING O? TANIZATION - NAME AND ADDRESS /If NRC, type "Same as above"; if co. "actor, provide N ZC Division, Office or Region, U.S. Nuclear Regufatory Commission, and mailing address.

Division of Regulatory Applications

Office of Nuclear Regulatory Research

U.S. Nuclear Regulatory Commission

Washington, DC 20555-0001

10. SUPPLEMENTARY NOTES

C. R. Mattsen, NRC Project Manager

11. ABSTRACT (200 words or less)

The feasibility of applying and adapting a two-dimensional laser heated thermoluminescence dosimetry system to the problem of surveying for radioactive surface contamination was studied. The system consists of a $\mathrm{CO}_{2}$ laser-based reader and monolithic arrays of thin dosimeter elements. The arrays consist of 10,201 thermoluminescent phosphor elements of 40 micron thickness, covering a $900 \mathrm{~cm}^{2}$ area. Array substrates are 125 micron ihick polyimide sheets, enabling them to easily coniorm to regular surface shapes, especially for survey of surfaces that are inaccessible for standard survey instruments. The passive, integrating radiation detectors are sensitive to alpha and beta radiation at contamination levels below release guideline limits. Required contact times with potentially contaminated surfaces are under one hour to achieve detection of transuranic alpha emission at $100 \mathrm{dpm} / 100 \mathrm{~cm}^{2}$. Positional information obtained from array evaluation is useful for locating contamination zones. Unique capabilities of this system for survey of sites, facilities and material include measurement inside pipes and other geometrical configurations that prevent standard surveys, and below-surface measurement of alpha and beta emitters in contaminated soils. These applications imply a reduction of material that must be classified as radioactive waste by virtue of its possibility of contamination, and cost savings in soil sampling at contaminated sites.

12. KEY WORDS/DESCR!PTORS /List words or phrases that will assist researchers in locating the report. I

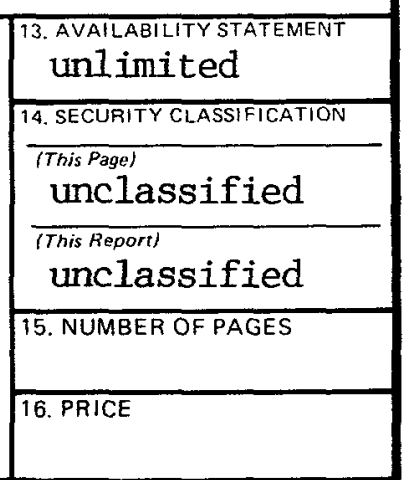

